\title{
Tuning the Electrical and Thermal Conductivities of Thermoelectric Oxides through Impurity Doping
}

\author{
Maria A. Torres Arango \\ West Virginia University
}

Follow this and additional works at: https://researchrepository.wvu.edu/etd

\section{Recommended Citation}

Torres Arango, Maria A., "Tuning the Electrical and Thermal Conductivities of Thermoelectric Oxides through Impurity Doping" (2013). Graduate Theses, Dissertations, and Problem Reports. 5007. https://researchrepository.wvu.edu/etd/5007

This Thesis is protected by copyright and/or related rights. It has been brought to you by the The Research Repository @ WVU with permission from the rights-holder(s). You are free to use this Thesis in any way that is permitted by the copyright and related rights legislation that applies to your use. For other uses you must obtain permission from the rights-holder(s) directly, unless additional rights are indicated by a Creative Commons license in the record and/ or on the work itself. This Thesis has been accepted for inclusion in WVU Graduate Theses, Dissertations, and Problem Reports collection by an authorized administrator of The Research Repository @ WVU. For more information, please contact researchrepository@mail.wvu.edu. 
Tuning the Electrical and Thermal Conductivities of Thermoelectric Oxides through Impurity Doping

Maria A. Torres Arango

Thesis submitted to the

Statler College of Engineering and Mineral Resources

at West Virginia University

in partial fulfillment of the requirements

for the degree of

Master of Science

in

Mechanical and Aerospace Engineering

Xueyan Song, Ph.D., Chair

Ever Barbero, Ph.D.

Hailin Li, Ph.D.

Department of Mechanical and Aerospace Engineering

Morgantown, West Virginia

2013

Keywords: Oxide Ceramics; Transport Properties; Thermopower; Nanoinclusions

Copyright 2013: Maria A. Torres Arango 


\title{
ABSTRACT \\ Tuning the Electrical and Thermal Conductivities of Thermoelectric Oxides through Impurity Doping
}

\author{
Maria A. Torres Arango
}

Waste heat and thermal gradients available at power plants can be harvested to power wireless networks and sensors by using thermoelectric (TE) generators that directly transform temperature differentials into electrical power. Oxide materials are promising for TE applications in harsh industrial environments for waste heat recovery at high temperatures in air, because they are lightweight, cheaply produced, highly efficient, and stable at high temperatures in air. $\mathrm{Ca}_{3} \mathrm{CO}_{4} \mathrm{O}_{9}$ (CCO) with layered structure is a promising $p$-type thermoelectric oxide with extrapolated ZT value of 0.87 in single crystal form [1]. However the ZT values for the polycrystalline ceramics remain low of $0.1-0.3$. In this research, nanostructure engineering approaches including doping and addition of nanoinclusions were applied to the polycrystalline CCO ceramic to improve the energy conversion efficiency.

Polycrystalline CCO samples with various $\mathrm{Bi}$ doping levels were prepared through the sol-gel chemical route synthesis of powders, pressing and sintering of the pellets. Microstructure features of $\mathrm{Bi}$ doped ceramic bulk samples such as porosity, development of crystal texture, grain boundary dislocations and segregation of Bi dopants at various grain boundaries are investigated from microns to atomic scale. The results of the present study show that the Bi-doping is affecting both the electrical conductivity and thermal conductivity simultaneously, and the optimum Bi doping level is strongly correlated with the microstructure and the processing conditions of the ceramic samples. At the optimum doping level and processing conditions of the ceramic samples, the Bi substitution of Ca results in the increase of the electrical conductivity, decrease of the thermal conductivity, and improvement of the crystal texture. The atomic resolution Scanning Transmission Electron Microscopy (STEM) Z-contrast imaging and the chemistry analysis also reveal the Bi-segregation at grain boundaries of CCO polycrystalline samples. In order to further decrease the thermal conductivity and increase the overall energy conversion efficiency of ceramic samples. The highest ZT value obtained is 0.32 at $973 \mathrm{~K}$ for Ca and Co site Bi doping. The effect of the nanoinclusions on the performance and the microstructure of CCO were investigated as well. 


\section{To my Family}




\section{Contents}

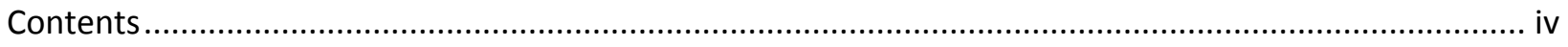

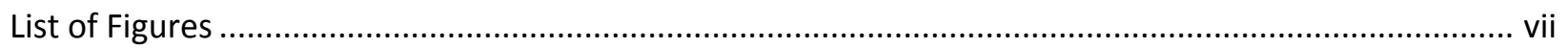

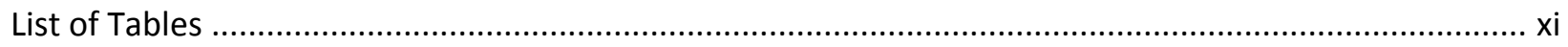

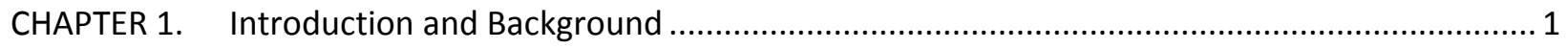

1.1 Potentials for the Application of Thermoelectric Materials ................................................. 1

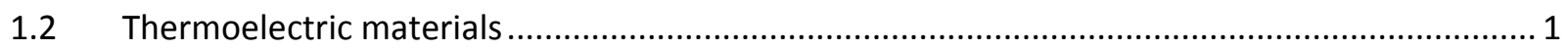

1.2.1 Seebeck Effect, Power Generation and Figure of Merit ZT............................................. 1

1.2.2 Conventional TE Materials: Properties, Advantages and Disadvantages .......................... 4

1.2.3 Oxide Ceramics: Uniqueness and Challenges ….................................................... 5

1.2.4 Doping and Nanoinclusions: Principles and Effect..................................................... 7

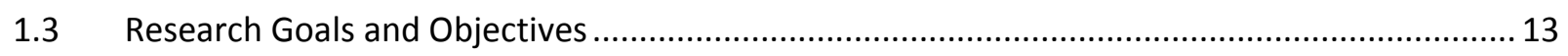

CHAPTER 2. Principles for the Materials Synthesis of Thermoelectric Materials and Thermoelectric

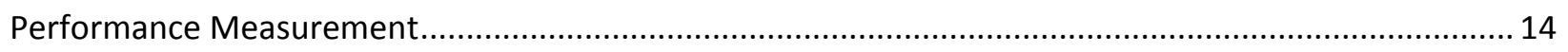

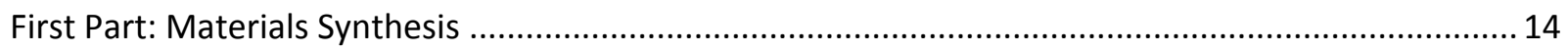

2.1 Synthesis Methods for Ceramics: Powder Samples ........................................................... 14

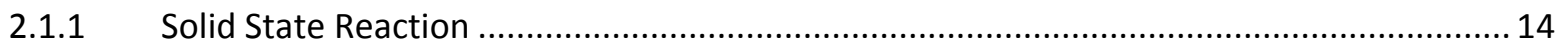

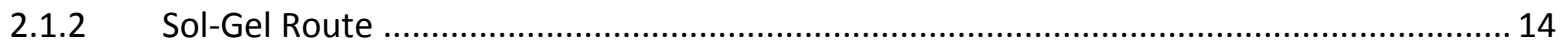

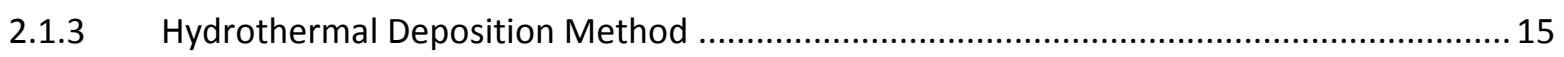

2.2 Sample Densification (Pellets Preparation) …............................................................. 15

Second Part: Thermoelectric Performance Measurement ....................................................... 17

2.3 Methods for Measuring Transport Properties: Seebeck Coefficient, Electrical Conductivity and

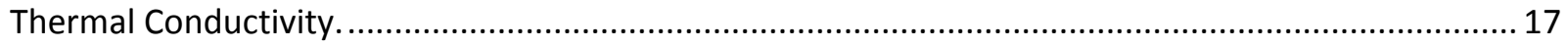

2.3.1 Seebeck Coefficient and Electrical Conductivity ....................................................... 18

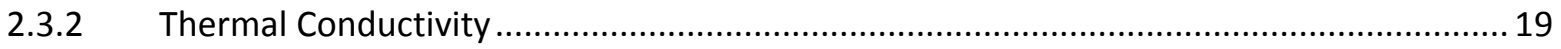

Third Part: Nanostructure and Chemistry Characterization using Scanning TEM (STEM)...................20 


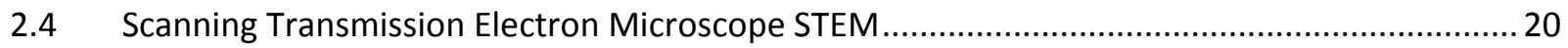

CHAPTER 3. Effect of Bi Doping on the Ca-site $\left(\mathrm{Ca}_{3-\mathrm{x}} \mathrm{Bi}_{x} \mathrm{Co}_{4} \mathrm{O}_{9}\right)$ on the Thermoelectric Performance and Nanostructure

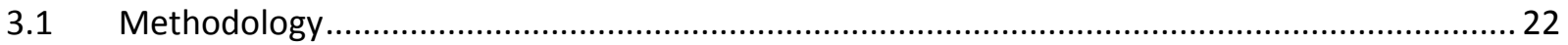

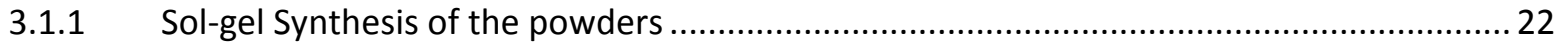

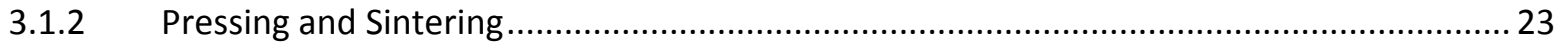

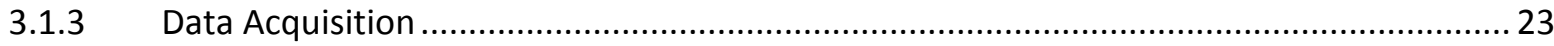

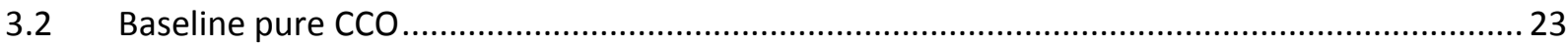

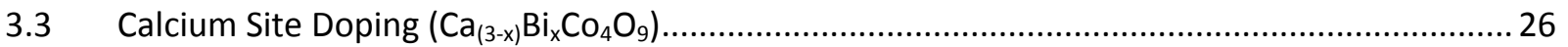

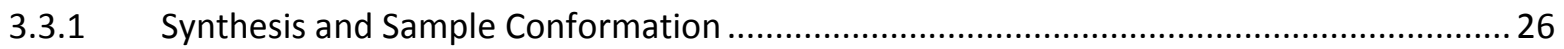

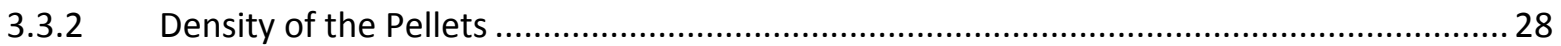

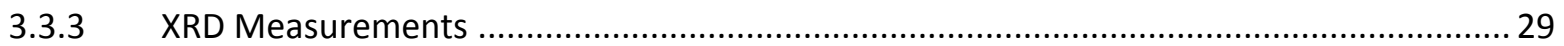

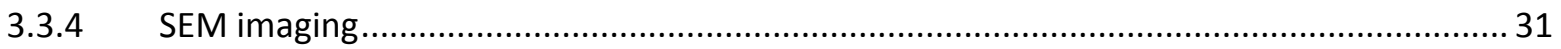

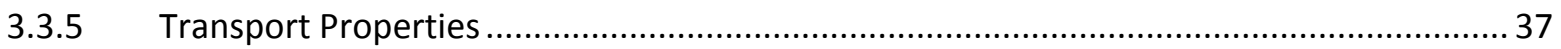

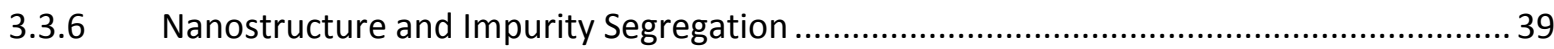

3.4 Effect of Pressing Conditions on Bi doped samples ............................................................ 46

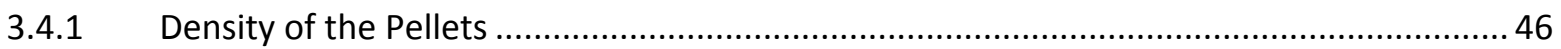

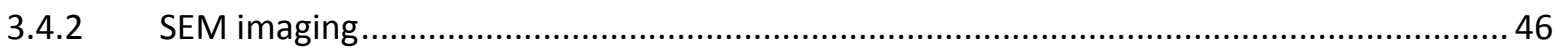

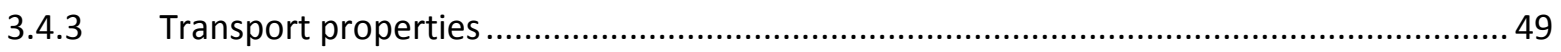

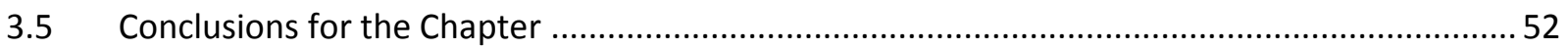

CHAPTER 4. Effect of Bi Doping on the Ca and Co Sites $\left(\mathrm{Ca}_{(3-x)} \mathrm{Bi}_{(x+y)} \mathrm{Co}_{(4-y)} \mathrm{O}_{9}\right)$ on the Thermoelectric

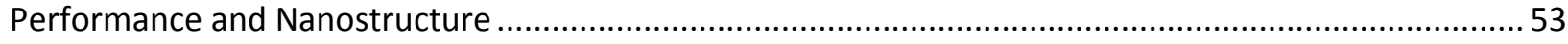

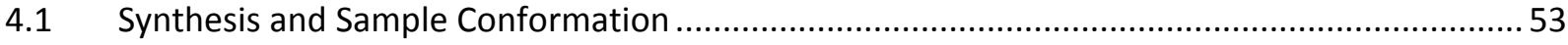

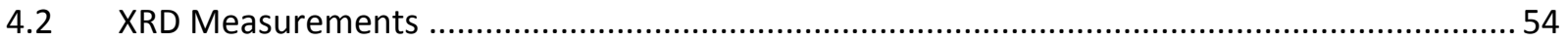

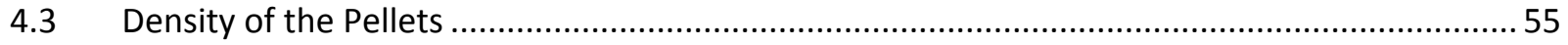

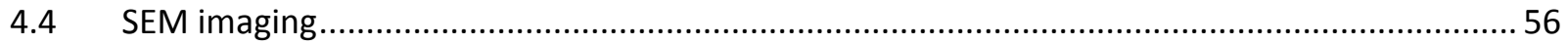




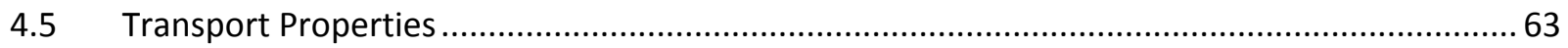

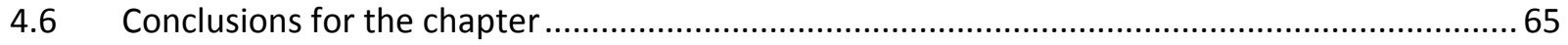

CHAPTER 5. Effect of Au Nanoinclusions on the Thermoelectric Performance and Nanostructure of

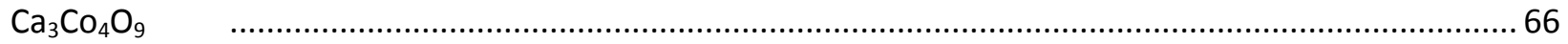

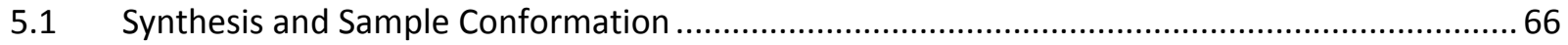

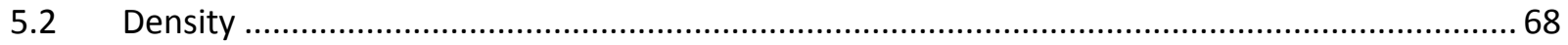

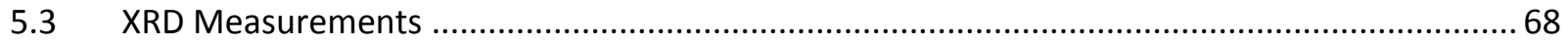

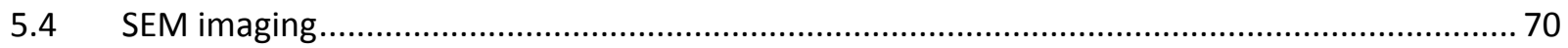

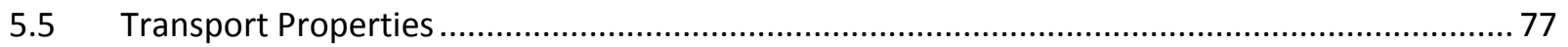

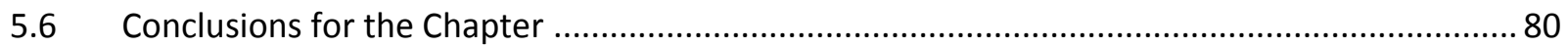

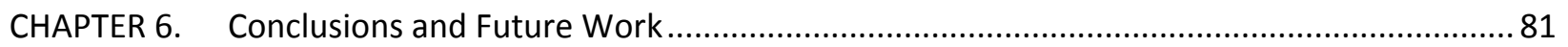

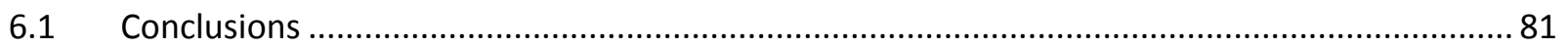

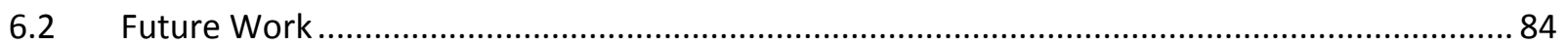

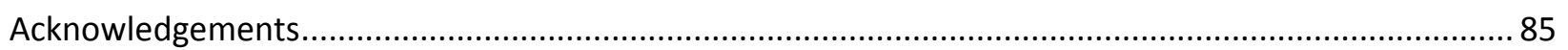

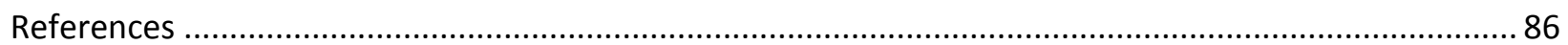




\section{List of Figures}

Figure 1. Schematic Seebeck and Peltier machines.[4] .................................................................... 2

Figure 2. Dependence of $\alpha, \sigma$ and $\rho$ on carrier concentration $n$. [4] ................................................... 3

Figure 3. Operating temperature and classification of thermoelectric materials.[6].............................. 4

Figure 4. Figure of merit $(Z)$ for traditionally used thermoelectric materials. [4] .................................... 5

Figure 5. ZT values for $p$-type thermoelectric materials performance and operation range.[1, 4, 10]...... 6

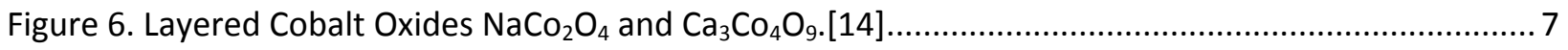

Figure 7. SEM micrographs of the fractured cross sections of CCO specimens processed by (a) conventional sintering, (b) hot pressing and (c)spark plasma sintering.[18] .......................................... 8

Figure 8. Figure of merit ZT for doped CCO. References in image correspond to source numeration.[50]

Figure 9. BSE images of fractured sections of CCO/Ag composites (a)5wt\%, (b) $10 w t \%$, (c) $20 w t \%$ and SEM (d) 20wt\%. Ag metallic phase is circled in (c) .[47]

Figure 10. Strategies for improvement of TE performance of polycrystalline CCO. (a) lamellar nanostructures, (b) preferentially oriented lamellar nanostructures, (c) oriented lamellar nanostructures with heavy element doping and (d) preferentially oriented lamellar nanostructures with heavy element

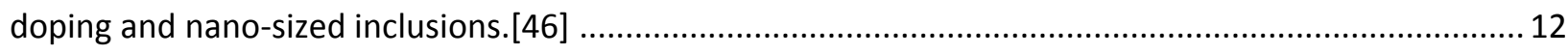

Figure 11. Schematic setting for hot (cold in absence of heat) pressing. [57]. ..................................... 16

Figure 12. Schematic setting of spark plasma sintering. [58] ........................................................... 17

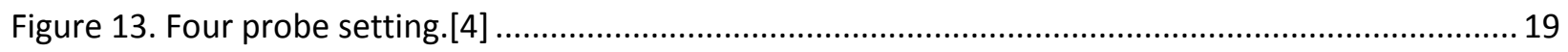

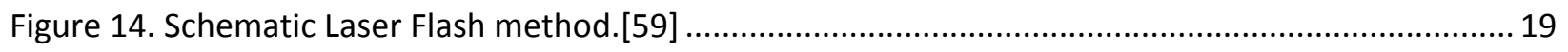

Figure 15. Schematic configuration and major elements of a scanning transmission electron microscope

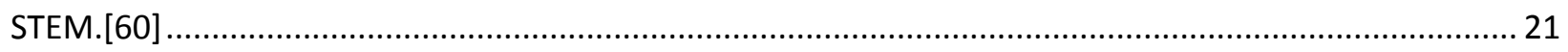

Figure 16. SEM image of CCO baseline powders after calcination at $650^{\circ} \mathrm{C}$ for 4 hours.........................24

Figure 17. TEM images of the baseline CCO powders after calcination at $650^{\circ} \mathrm{C}$ for 4 hours...................24

Figure 18. SEM images of Bi doped CCO powders after calcination at $650^{\circ} \mathrm{C}$ for 4 hours........................2 27

Figure 19. TEM images of $\mathrm{Bi} 0.3$ doped powders after calcination at $650^{\circ} \mathrm{C}$ for 4 hours.........................28

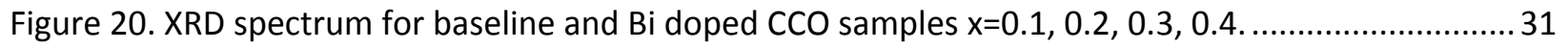

Figure 21. Pressed plane of baseline and Bi doped CCO pellets. Pressed at $1 \mathrm{GPa}$ and $150^{\circ} \mathrm{C}$ for 40 minutes. 
Figure 22. Pressed plane of baseline and Bi dopped CCO pressed at $1 \mathrm{GPa}$ and $150^{\circ} \mathrm{C}$ (lower magnification). 33

Figure 23. Cross section of baseline and Bi doped CCO pellets. Pressed at $1 \mathrm{GPa}$ and $150^{\circ} \mathrm{C}$ for 40 minutes. In plane direction coincides with horizontal direction, perpendicular to pressing axis. .35

Figure 24. TEM image and diffraction pattern along the [110] zone axis for the Bi 0.3 doped samples. ...36 Figure 25. TEM image and diffraction pattern along the $\left[1 \overline{2}^{2}\right.$ ] zone axis for the Bi 0.4 doped samples...37 Figure 26. Transport properties for the baseline and Bi doped CCO samples $x=0.1,0.2,0.3$ and 0.4 Pressed at $1 \mathrm{GPa}$ and $150^{\circ} \mathrm{C}$.

Figure 27. Dimensionless figure of merit for the baseline and Bi doped CCO samples $x=0.1,0.2,0.3,0.4$.

Figure 28. STEM image of CCO - Bi 0.3 doped sample showing Bi segregation features in grain boundaries type I and II.

Figure 29. STEM images of CCO - Bi 0.3 doped samples pressed at $1 \mathrm{GPa}$. Bi segregation occurs at the boundaries between lamellae. Type II grain boundary is off axis.

Figure 30. Type I grain boundary dislocation repeating unit, (a) Bi depleted region (dark contrast line), (b) Bi enriched region (bright contrast line).

Figure 31. High resolution STEM images of the Bi depleted (a) and enriched (b) regions of the dislocations found in type I grain boundary.

Figure 32. TEM images of the grain boundary Type II on axis. (a) Grain boundary with diffraction pattern of the circled area. (b) High resolution TEM image of the dislocations present between lamellae. .45

Figure 33. Pressed plane of baseline and Bi doped CCO pellets. Pressed at $0.5 \mathrm{GPaand} 25^{\circ} \mathrm{C}$ for 40 minutes.

Figure 34. Pressed plane of baseline and Bi dopped CCO pressed at $0.5 \mathrm{GPa}$ and $25^{\circ} \mathrm{C}$ (lower magnification).

Figure 35. Cross section of baseline and Bi doped CCO pellets. Pressed at $0.5 \mathrm{GPa}$ and $25^{\circ} \mathrm{C}$ for 40 minutes. In plane direction coincides with horizontal direction, perpendicular to pressing axis.

Figure 36. Transport properties for the baseline and Bi doped CCO samples $x=0.1,0.2$ and 0.3. Pressed at $25^{\circ} \mathrm{C}$ and $0.5 \mathrm{GPa}$ for 40 minutes. 50

Figure 37. Dimensionless figure of merit for the baseline and Bi doped CCO samples $x=0.1,0.2$ and 0.3. Pressed at $25^{\circ} \mathrm{C}$ and $0.5 \mathrm{GPa}$ for 40 minutes. 51 Figure 38. SEM image of CCO - Bi doped on $\mathrm{Ca}$ and $\mathrm{Co}$ sites powders after calcination at $650^{\circ} \mathrm{C}$ for 4 hours. 53 
Figure 39. XRD Spectrum of CCO-Bi $x=0.2, y=0.1$ doped system.

Figure 40. Pressed planes and cross sectional SEM images of CCO - Bi doped Ca $x=0.2$, Co y=0.1 levels at 0.5 and $1 \mathrm{GPa}$. For cross section images, in plane direction coincides with horizontal direction, perpendicular to pressing axis.

Figure 41. TEM image and diffraction pattern of circled area of CCO - Bi ( $\mathrm{Ca} x=0.2, y=0.1)$ doped sample pressed at $0.5 \mathrm{GPa}$ and $25^{\circ} \mathrm{C}$. 58

Figure 42. High resolution TEM image of grain boundary Type I with dislocation. CCO (Ca $x=0.2, y=0.1)$ doped sample pressed at $0.5 \mathrm{GPa}$ and $25^{\circ} \mathrm{C}$.

Figure 43. SEM images of the pressed plane for a sample pressed at $1 \mathrm{GPa}$ and $150^{\circ} \mathrm{C}$. Differences in the microstructure features are identified. 60 Figure 44 . SEM images of the cross section for a sample pressed at $1 \mathrm{GPa}$ and $150{ }^{\circ} \mathrm{C}$. Differences in the microstructure features are identified.

Figure 45. Transport properties for the $\mathrm{Ca}$ and $\mathrm{Co}$ site $\mathrm{Bi}$ doped $\mathrm{CCO}$ samples pressed at $0.5 \mathrm{GPa}-25^{\circ} \mathrm{C}$ and $1 \mathrm{GPa}-150^{\circ} \mathrm{C}$.

Figure 46. Dimensionless figure of merit for the $\mathrm{Ca}$ and $\mathrm{Co}$ site $\mathrm{Bi}$ doped CCO samples pressed at $0.5 \mathrm{GPa}$

$-25^{\circ} \mathrm{C}$ and $1 \mathrm{GPa}-150^{\circ} \mathrm{C}$ 64

Figure 47. SEM image of CCO - Au $10 \%$ powders after hydrothermal deposition and drying at $300^{\circ} \mathrm{C}$ for 4 hours. 67

Figure 48. TEM images of the CCO - Au10\% powders after hydrothermal deposition. 67

Figure 49. XRD spectrum for baseline and CCO - Au\% samples.Au concentrations of $1 \%, 2 \%, 5 \%$ and $10 \%$.

Figure 50. Pressed plane of baseline and Au composite CCO pellets. Pressed at $1 \mathrm{Gpa}$ and $150^{\circ} \mathrm{C}$ for 40 minutes.

Figure 51. Pressed plane of baseline and CCO - Au nanoinclusionsadded samples pressed at $1 \mathrm{GPa}$ and $150^{\circ} \mathrm{C}$ (lower magnification).

Figure 52. Pressed plane of baseline and CCO - Au $10 \mathrm{wt} \%$ nanoinclusions added samples pressed at 1 $\mathrm{GPa}$ and $150^{\circ} \mathrm{C}$ (lower magnification).

Figure 53. Cross section of baseline and Au composite CCO pellets.Pressed at $1 \mathrm{Gpa}$ and $150^{\circ} \mathrm{C}$ for 40 minutes. In plane direction coincides with horizontal direction, perpendicular to pressing axis............. 74 Figure 54. TEM images of the CCO - Au 1\% composite samples. 75 Figure 55. High resolution TEM images of the CCO - Au1\% samples. (a) diffraction pattern of marked area in (b) only CCO; the diffraction pattern is taken along the [1 $\overline{2}_{0}$ ] zone axis. (c) Au nanoparticle coherent 
with the CCO matrix. (d) diffraction pattern of the Au nanoparticle and CCO matrix taken from the

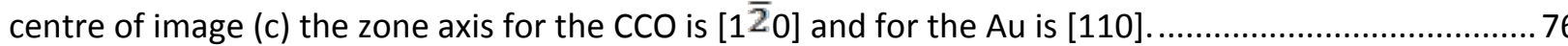
Figure 56. Transport properties for the baseline and CCO - Au \% composites. Au wt\% concentrations of $1 \%, 2 \%, 5 \%$ and $10 \%$.

Figure 57. Dimensionless figure of merit for the baseline and CCO - Au \% composites. Au wt\% concentrations of $1 \%, 2 \%, 5 \%$ and $10 \%$.

Figure 58. Dimensionless figure of merit for the best performing CCO materials among the systems studied. 


\section{List of Tables}

Table 1. Precursor constituents of Sol-gel CCO and CCO-doped/nanoinclusions added systems. ............ 22

Table 2. Density of CCO systems, baseline and Bi doped series.............................................................29

Table 3. Powder X-Ray diffraction indexed peaks of CCO Baseline and Bi x=0.1 to 0.4 doped systems....29

Table 4. EDS analysis of the Bi segregation regions as depicted in Figure 29. ....................................... 41

Table 5. EDS analysis of the dislocation regions (a) and (b) in Figure 31, grain boundary type I.............. 44

Table 6. Density values for the CCO system, baseline and Bi doped series pressed at $0.5 \mathrm{GPa}$ and $25^{\circ} \mathrm{C} .46$

Table 7. Powder X-Ray diffraction indexed peaks of $\mathrm{CCO}$ Baseline and $\mathrm{Bi}(\mathrm{Ca} \mathrm{x}=0.2, \mathrm{Co} \mathrm{y}=0.1)$ doped

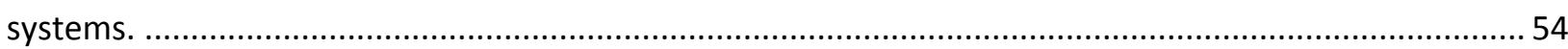

Table 8. Density values for the CCO system, baseline and selective site Bi doped samples pressed at 0.5

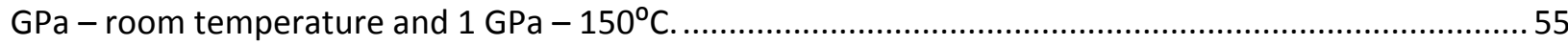

Table 9. EDS analysis of CCO - Bi ( $\mathrm{Ca} x=0.2, \mathrm{y}=0.1)$ 1GPa sample with different microstructure features as

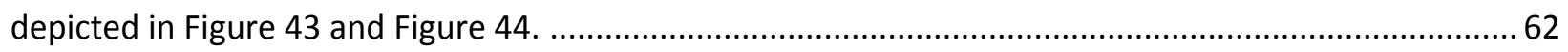

Table 10. Density of CCO systems, baseline and Au composite series...................................................68

Table 11. Powder X-Ray diffraction indexed peaks of CCO Baseline and CCO - Au wt \% added systems. . 69 


\section{CHAPTER 1. Introduction and Background}

\subsection{Potentials for the Application of Thermoelectric Materials}

Thermoelectric (TE) materials are materials that show thermoelectric behavior, this is, Seebeck or Peltier effects when exposed to heat or voltage gradients respectively. Conversion of heat to electrical power using TE systems requires no moving parts and emits no residual $\mathrm{CO}_{2}$ gas or other hazardous substances. Also the possibility to use thermoelectric materials at different scale size makes this technology even more versatile, allowing for powering of nano-sized applications as well as micro and macro systems.

\subsection{Thermoelectric materials}

\subsubsection{Seebeck Effect, Power Generation and Figure of Merit ZT}

The Seebeck effect is the generation of an electromotive force due to the heating of the junction of two different conducting materials. This effect was first observed by J. T. Seebeck in 1821. The opposite is the Peltier effect, the induction of a current from one material to another alters the energy transported by the electrons showing heating or cooling at the interface between the materials.[2].

The power generation takes place when the Seebeck effect is applied to obtain voltage from heat. The schematic machine is shown in Figure 1, it is composed of two different thermoelectric materials, one is $\mathrm{n}$-type and the other is a $\mathrm{p}$-type semiconductor.

Heat form the hot (waste) source will create a temperature gradient with the cold heat sink giving rise to a differential of potential. The materials will be connected at the cold heat sink by a load. The heat will induce a current flow through the circuit delivering electrical power at the load.[3] 


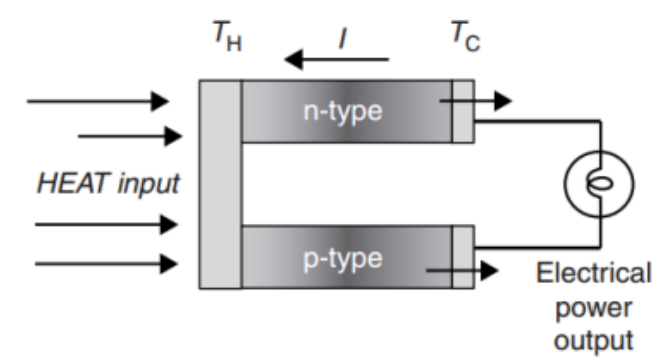

The Seebeck effect (Thermoelectric generation)

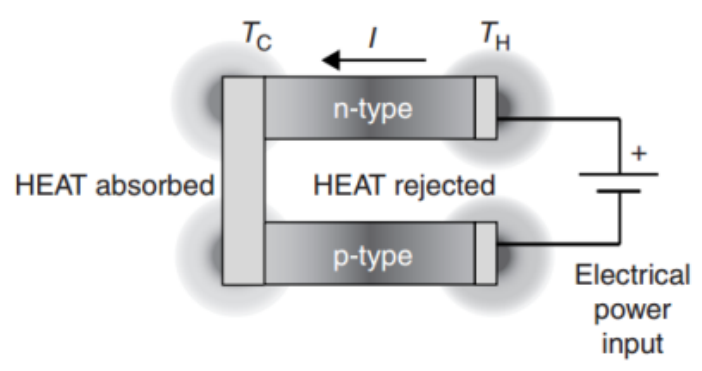

The Peltier effect (Thermoelectric cooling)

Figure 1. Schematic Seebeck and Peltier machines.[4]

The transport properties of a thermoelectric material are the thermoelectric coefficients (Seebeck and Peltier), the thermal and the electrical conductivities. These properties are used to estimate the performance of a thermoelectric material through the figure of merit $Z$ or the dimensionless figure of merit ZT.

$$
Z=\frac{\alpha^{2} \sigma}{\lambda}=\frac{\alpha^{2} \sigma}{\lambda_{e l}+\lambda_{L}}
$$

Where $\alpha$ is the Seebeck coefficient, $\sigma$ and $\lambda$ are the electrical and thermal conductivities respectively. The thermal conductivity is composed by two main contributions: the carrier component $\lambda_{\mathrm{el}}$ and the lattice component $\lambda_{\mathrm{L}}[4]$. When $\mathrm{Z}$ is multiplied by the absolute temperature $\mathrm{T}$ in Kelvin, the dimensionless figure of merit is obtained. The efficiency reaches $\sim 10 \%$ at $Z T=1$, and $Z T \geq 1$ is generally accepted as a criterion for practical applications[4].

From Equation 1, it can be seen that by increasing the electrical conductivity and the Seebeck coefficient and decreasing the thermal conductivity, larger values for $Z$ are to be expected.

All the transport properties are dependent on the carrier concentration $n$; both the thermal and electrical conductivities increase as the carrier concentration increases, whereas the Seebeck coefficient 
decreases with increasing carrier concentration.[5] Figure 2 illustrates this condition. The performance of a given thermoelectric material is also temperature dependent.

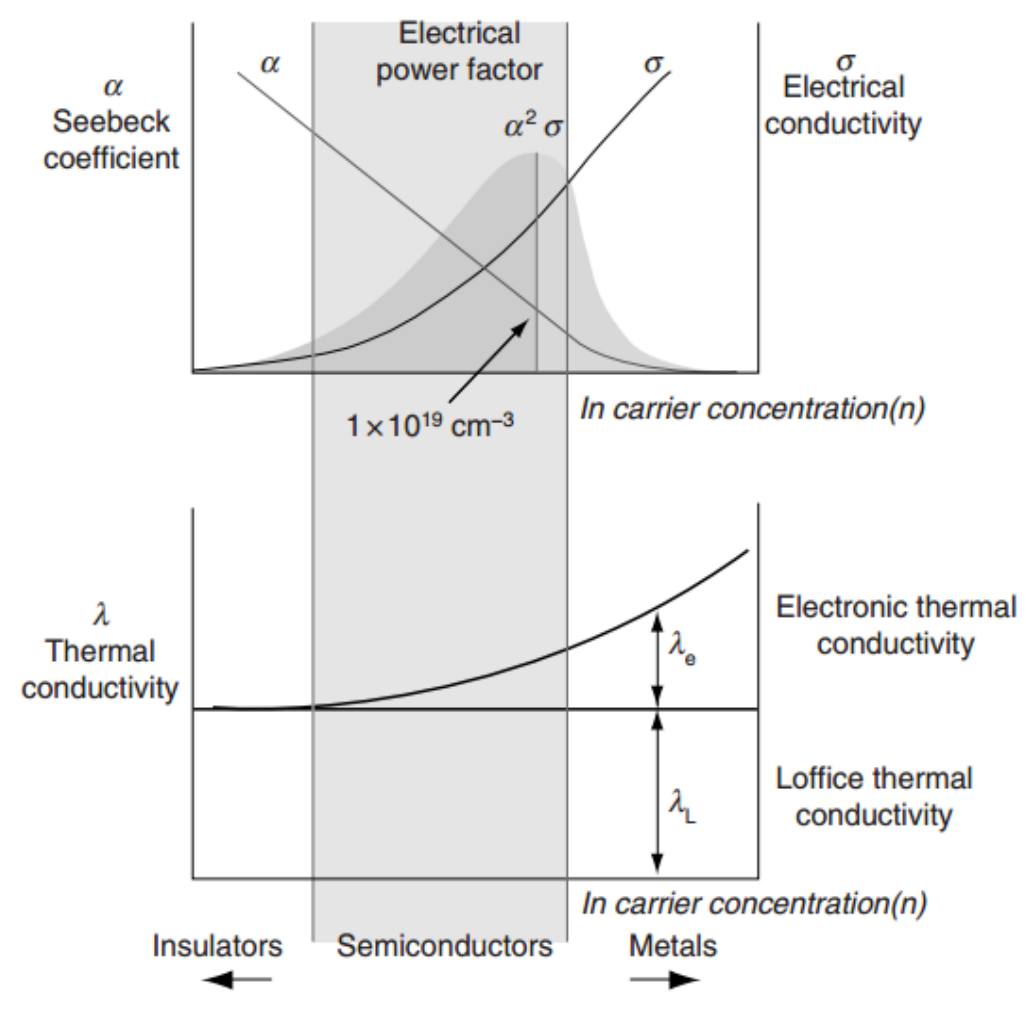

Figure 2. Dependence of $\alpha, \sigma$ and $\rho$ on carrier concentration $n$. [4]

The lattice component of the thermal conductivity $\lambda_{L}$, not only does not depend on the carrier concentration but also limits the lowest threshold for the overall conductivity; aiming to its reduction will lead to the smallest value for this transport property. This component is associated to phonon scattering and decreases as the atomic weight increases; therefore it is the main reason for the use of heavy elements such as $\mathrm{Bi}, \mathrm{Te}, \mathrm{Ge}$ and $\mathrm{Pb}$ as traditional thermoelectric materials.[6] 


\subsubsection{Conventional TE Materials: Properties, Advantages and Disadvantages}

Temperature gradients such as those developed from industrial furnaces and exhaust gases (in industry and transport field) are considered waste heat. Great effort is being used in isolation of these hot surfaces to achieve higher efficiency of the machinery and also because of the risk they represent to people and the environment. Thermoelectric materials can further transform this thermal energy into electrical power; however conventional TE materials are toxic and unstable at high temperatures limiting their applications for high temperature ranges, confinement to avoid release of dangerous gases and particulate material upon decomposition is required.

Conventional TE materials are classified upon their operation range; low temperature (up to 450K) are generally used in refrigeration applications and consist of intermetallic compounds made of $\mathrm{Bi}$ with $\mathrm{Sb}$, Te and Se. Intermediate temperature consist of $\mathrm{Pb}$-Te compounds (up to 850K) and high temperature (up to $1300 \mathrm{~K}$ ) thermoelectric materials which are mainly Si-Ge alloys.[4] Detailed information is found in Figure 3 and Figure 4.

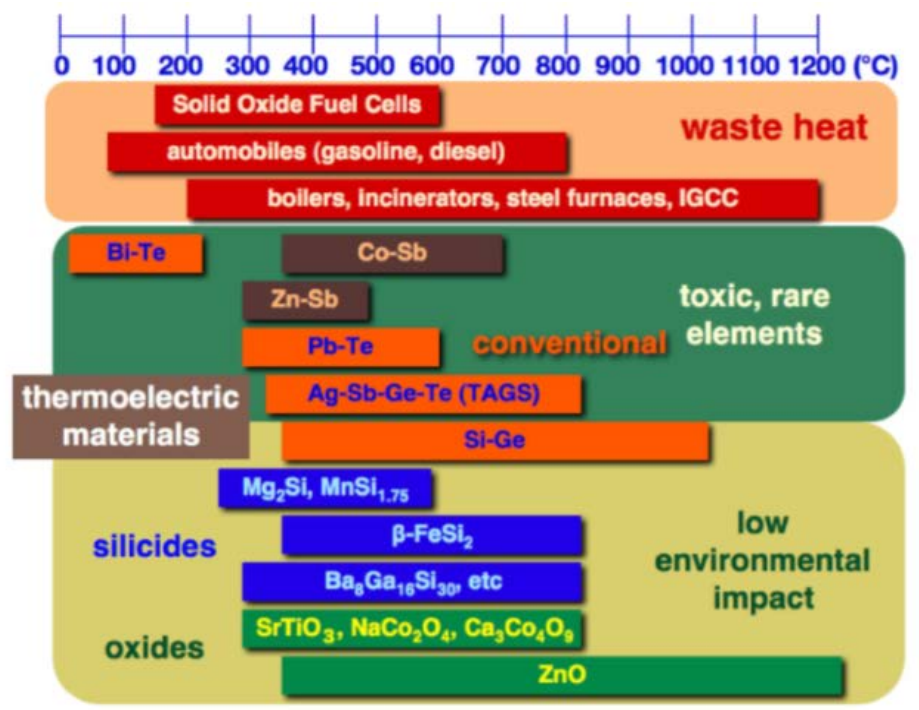

Figure 3. Operating temperature and classification of thermoelectric materials.[6]

The materials used traditionally for thermoelectric power generation are bimetallic compounds such as bismuth telluride (ZT of $\approx 1.1$ at $330 \mathrm{~K}$ ), lead telluride (ZT of $\approx 0.7$ at $740 \mathrm{~K}$ ) and silicon germanium (ZT of $\approx 0.5$ at 1100K).[4] Detailed information can be found in Figure 4 and Figure 5. 


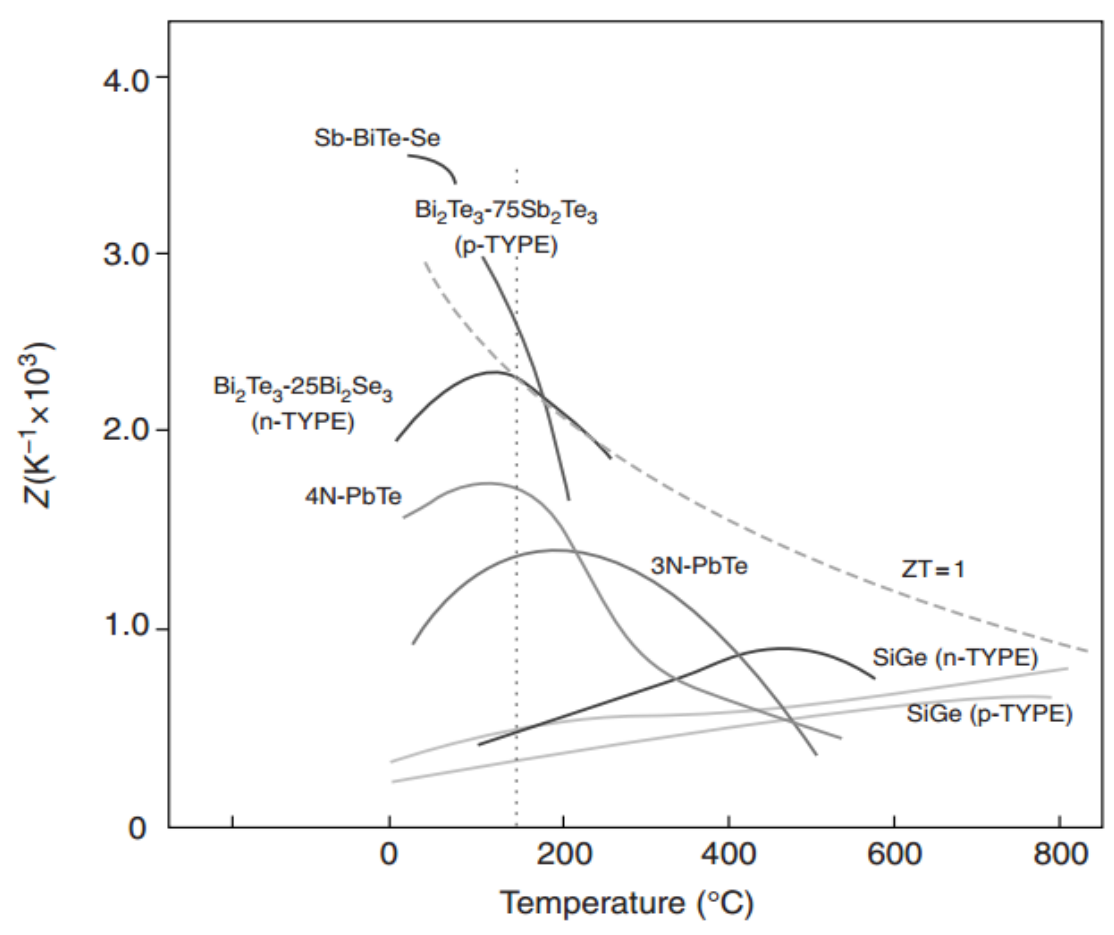

Figure 4. Figure of merit (Z) for traditionally used thermoelectric materials. [4]

The main disadvantages for the use of traditional materials are the high cost, toxicity, chemical instability at temperatures above 300K.

\subsubsection{Oxide Ceramics: Uniqueness and Challenges}

Metal oxides are compounds of ionic nature where the cations are generally transition metals and oxygen atoms act as the anions. These oxide ceramics are used for thermoelectric power generation as a good alternative because they are already oxidized and thus stable enough for high temperature regimes (up to $1500 \mathrm{~K}$, see Figure 3 ). They are much less expensive, easy to manufacture and generally nontoxic. Although they exhibit lower values for $\mathrm{ZT}$, their performance as a whole makes them very attractive.

The most commonly used oxide ceramics are n-type Calcium Manganese Oxides $\left(\mathrm{CaMnO}_{3}\right)-\mathrm{CMO}^{\prime} \mathrm{s}$ (highest ZT of 0.32 [7]), p-type Calcium Cobalt Oxides - CCO's (highest ZT of 0.87 [1]), n-type Strontium Titanium Oxide $-\mathrm{SrTiO}_{3}$ (highest ZT of 2.4 [8]) and n-type Zinc Oxide - ZnO (highest ZT of 0.65 [9]). The high temperature stability of these oxides is their main advantage among the other materials. 


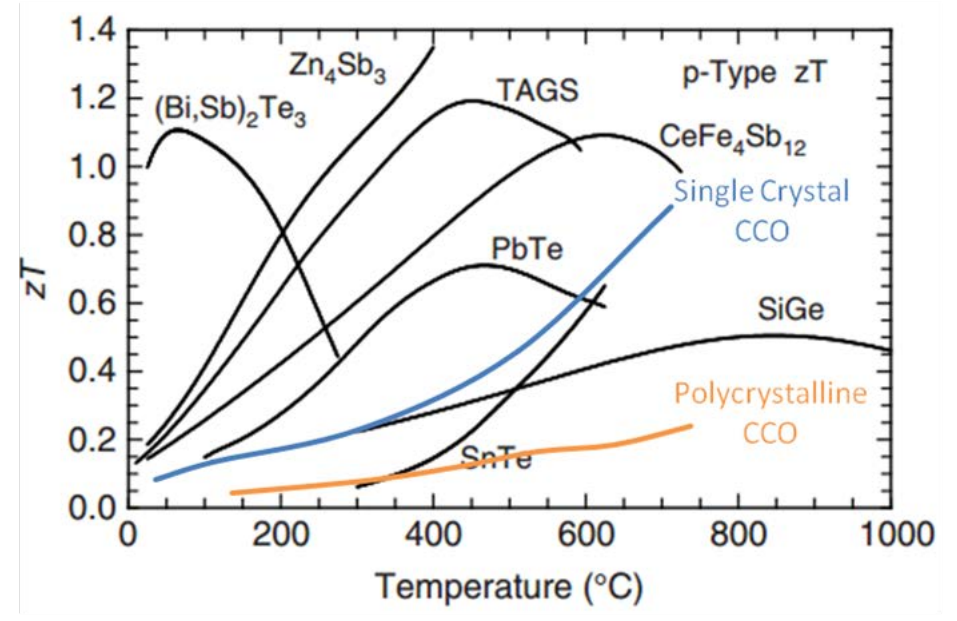

Figure 5. ZT values for p-type thermoelectric materials performance and operation range.[1, 4, 10].

Thermoelectric properties of $\mathrm{NaCO}_{2} \mathrm{O}_{4}[11]$ (highest $\mathrm{ZT}$ of about 1 [12]) were first studied by Terasaki in 1997[11] and since, attention was driven to the study of cobalt oxides. The poor stability of sodium limits the use of $\mathrm{NaCO}_{2} \mathrm{O}_{4}$ at high temperatures. The need for better performance of similar compounds such as CCO is then a vast field for research. Recently, the concept of nanoblock integration into layerstructured hybrid crystal as an approach to enhance ZT was proposed.[4] It is, layered oxides such as $\mathrm{Na}_{x} \mathrm{CoO}_{2}$ or $\mathrm{Ca}_{3} \mathrm{Co}_{4} \mathrm{O}_{9}$ possessing layered structures on which $\mathrm{CoO}_{2}$ layers are responsible for high conductivity whereas the $\mathrm{Na}$ or $\mathrm{Ca}_{2} \mathrm{CoO}_{3}$ nanoblocks act as phonon scattering regions and lead to low thermal conductivity.

Calcium Cobalt Oxide - CCO system is a misfit layered compound of the type $\left[\mathrm{Ca}_{2} \mathrm{CoO}_{3}\right] \mathrm{b}_{1} / \mathrm{b}_{2}\left[\mathrm{CoO}_{2}\right]$ where the $\mathrm{Ca}_{2} \mathrm{CoO}_{3}$ layers are constituted of a rock salt crystalline structure and the $\mathrm{CoO}_{2}$ layers are in $\mathrm{Cdl}_{2}$ crystalline structure; $\mathrm{b} 1$ and $\mathrm{b} 2$ are the respective periodicities for the two subsystems along the $\mathrm{b}$ axis. These layers are stacked along the $c$ axis in an alternating pattern.[13] Such an arrangement provides highly anisotropic transport properties: preferred electrical conductivity along the $a b$ plane through the $\mathrm{CoO}_{2}$ layers[13] and low thermal conductivity[13] at the layer interfaces. The compound structure can be observed in Figure 6. 

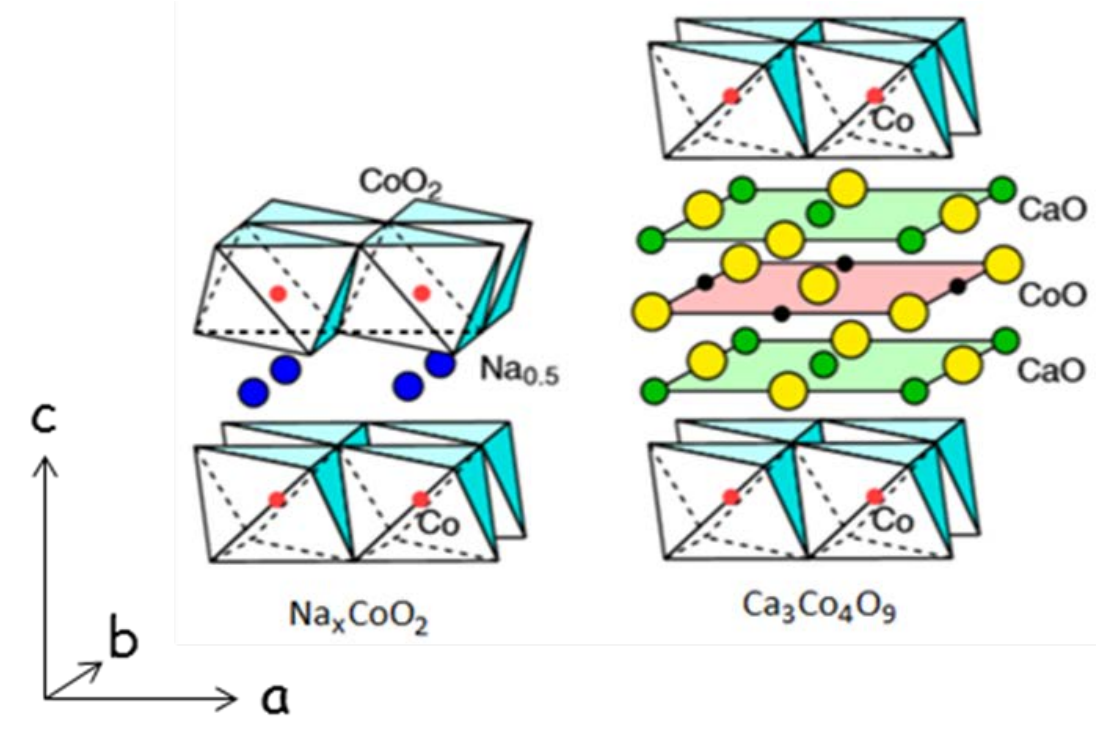

$$
\mathrm{Ca}_{3} \mathrm{CO}_{4} \mathrm{O}_{9}
$$

Figure 6. Layered Cobalt Oxides $\mathrm{NaCO}_{2} \mathrm{O}_{4}$ and $\mathrm{Ca}_{3} \mathrm{CO}_{4} \mathrm{O}_{9} \cdot[14]$

Thermoelectric properties differ for single crystal and polycrystalline materials. In general the ZT values are higher for single crystal, however it is too expensive to manufacture single crystals; polycrystalline materials come as a fair solution. In order to achieve single crystal like properties, the polycrystalline materials must aim to similar density and alignment of the grains. Highly crystalline textured materials can be obtained through high pressure sintering and spark plasma sintering. The highest value of ZT for single crystal CCO is 0.87 at $973 \mathrm{~K}[1]$ and for polycrystalline (Fe doped)CCO is 0.4 at 1000K[15]. A composite CCO made of single crystal $20 \mathrm{wt} \%$ and $80 \mathrm{wt} \%$ polycrystalline material was also reported to exhibit a ZT of 0.48 at $973 \mathrm{~K}[16]$.

\subsubsection{Doping and Nanoinclusions: Principles and Effect}

Enhancement and tuning of the performance of CCO is possible. Crystal texture development, doping and co-doping of the $\mathrm{Ca}$ and $\mathrm{Co}$ sites and the recent addition of nanometric size particles are the main approaches to do so.

The CCO being a layered thermoelectric material exhibits preferential TE behavior along the $a b$ planes of the compound (See Figure 6). In the polycrystalline samples the orientation degree is not as favorable as for the single crystal case; this is the main reason for the difference in the thermoelectric performance of the single crystal and the polycrystalline CCO materials. Single crystal - like texture development has been studied to achieve better performance for the polycrystalline specimens [1, 16-26]. These 
approaches include crystal texture development and densification of the materials. Strong alignment of the materials is achieved by uniaxial hot pressing as seen from Figure 7.
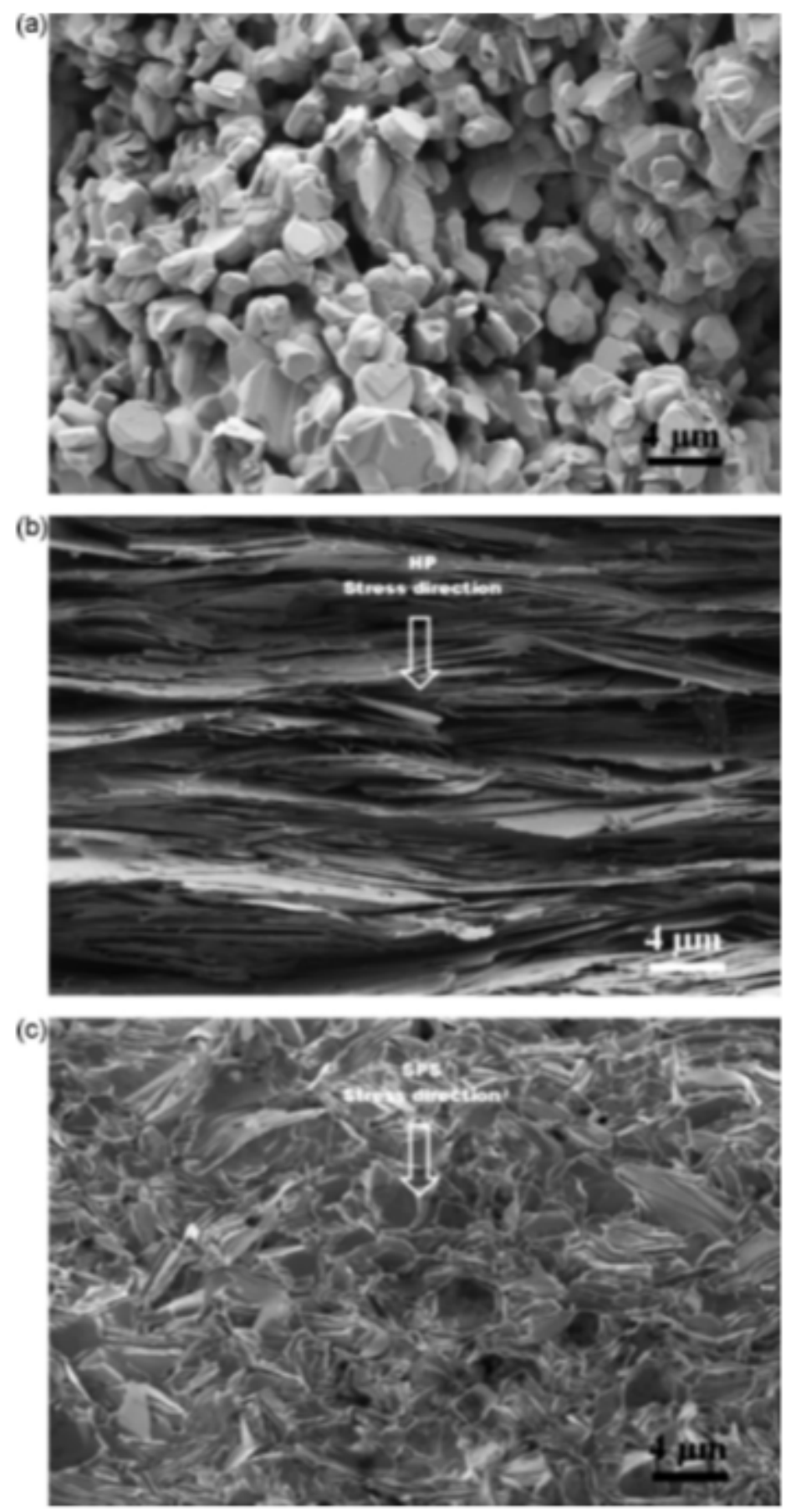

Figure 7. SEM micrographs of the fractured cross sections of CCO specimens processed by (a) conventional sintering, (b) hot pressing and (c)spark plasma sintering.[18] 
Doping of the CCO system with heavier elements has been done for the calcium site[10, 15, 16, 22, 24, 27-38] and for the cobalt site[15, 39, 40].Substitution of the Co sites in the $\mathrm{CoO}_{2}$ layers is expected to increase the charge carrier concentration by inducing oxygen vacancies and increasing the electrical conductivity.[15, 41] In general, the substitution doping with heavier atoms is expected to reduce the thermal conductivity.[42]Co-doping (simultaneous Co and Ca sites) has also been explored Bi[43], Gd$\mathrm{Y}[44]$ and lately, composite structures involving the inclusion of metallic particles [45-49]. This latter approach is expected to further reduce the thermal conductivity.

The doping approach on the CCO system intends to increase the electrical conductivity by increasing the carrier mobility rather than the carrier concentration as is the case of Bi doping. $[24,28,29,38,50]$ due that the increase in the carrier concentration has been identified to decrease the Seebeck coefficient. The addition of $\mathrm{Ag}$ as dopant and co-dopant with $\mathrm{Ba}$ and $\mathrm{Lu}$ is known to increase the electrical conductivity from both of the mechanisms described.[36,50] $\mathrm{Cu}$ doping in contrast with the previous occur for the Co sites, mainly in the $\mathrm{Ca}_{2} \mathrm{CoO}_{3}$ insulating layers, as a result the electrical conductivity is increased and the Seebeck coefficient is decreased.[15] The summary of the values of figure of merit ZT for the CCO is presented in Figure 8. 

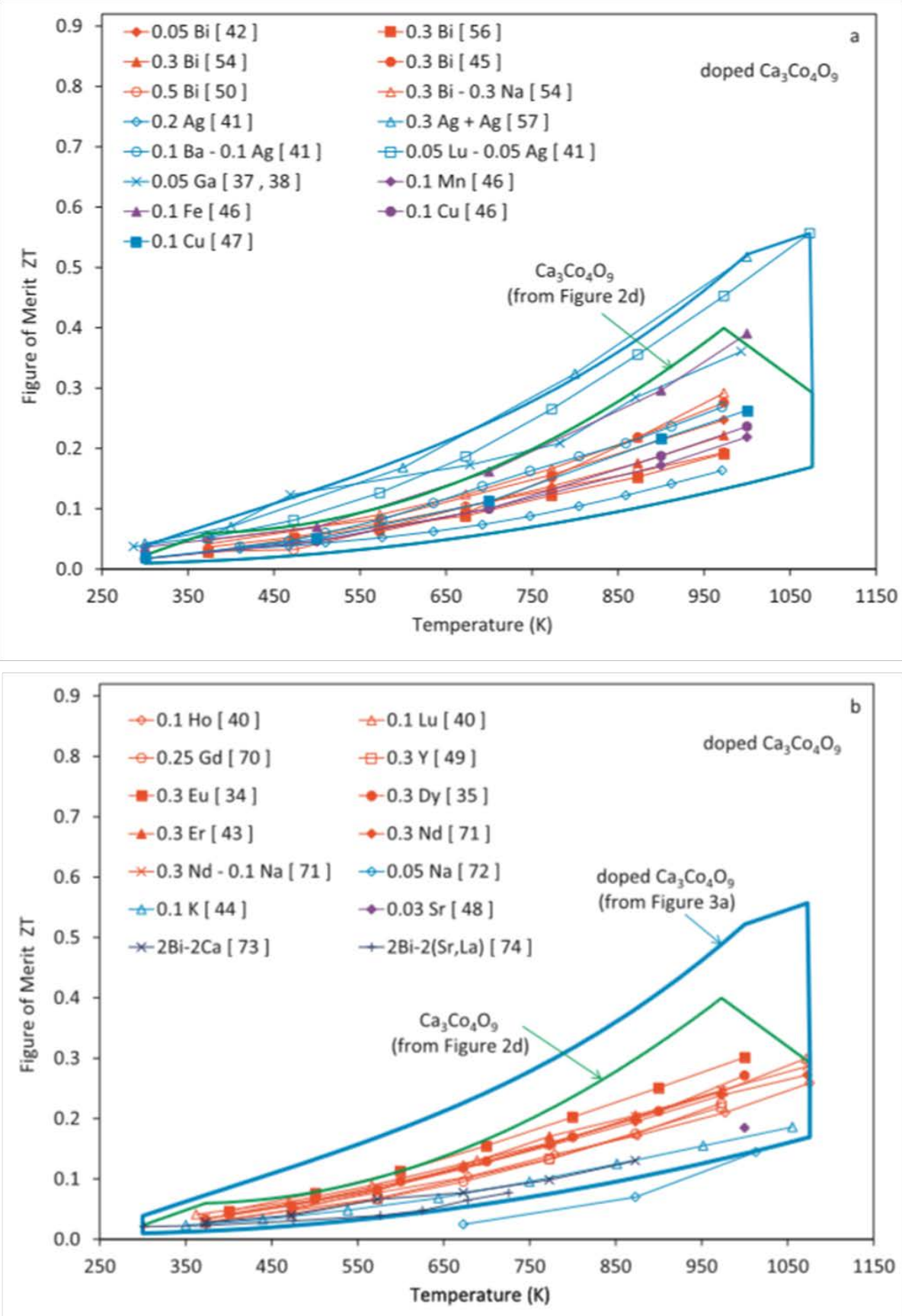

Figure 8. Figure of merit ZT for doped CCO. References in image correspond to source numeration.[50]

The addition of metallic $\mathrm{Ag}$ as a secondary phase to the CCO system has been reported to result in Seebeck coefficient reduction [36, 45, 47, 48, 50,51]; however combined with Ag as dopant the electrical conductivity and the thermopower are increased [50-52]. In Figure 9, Ag inclusions on the CCO are shown. Notice that the size of the Ag particles is comparable to the CCO grains. 

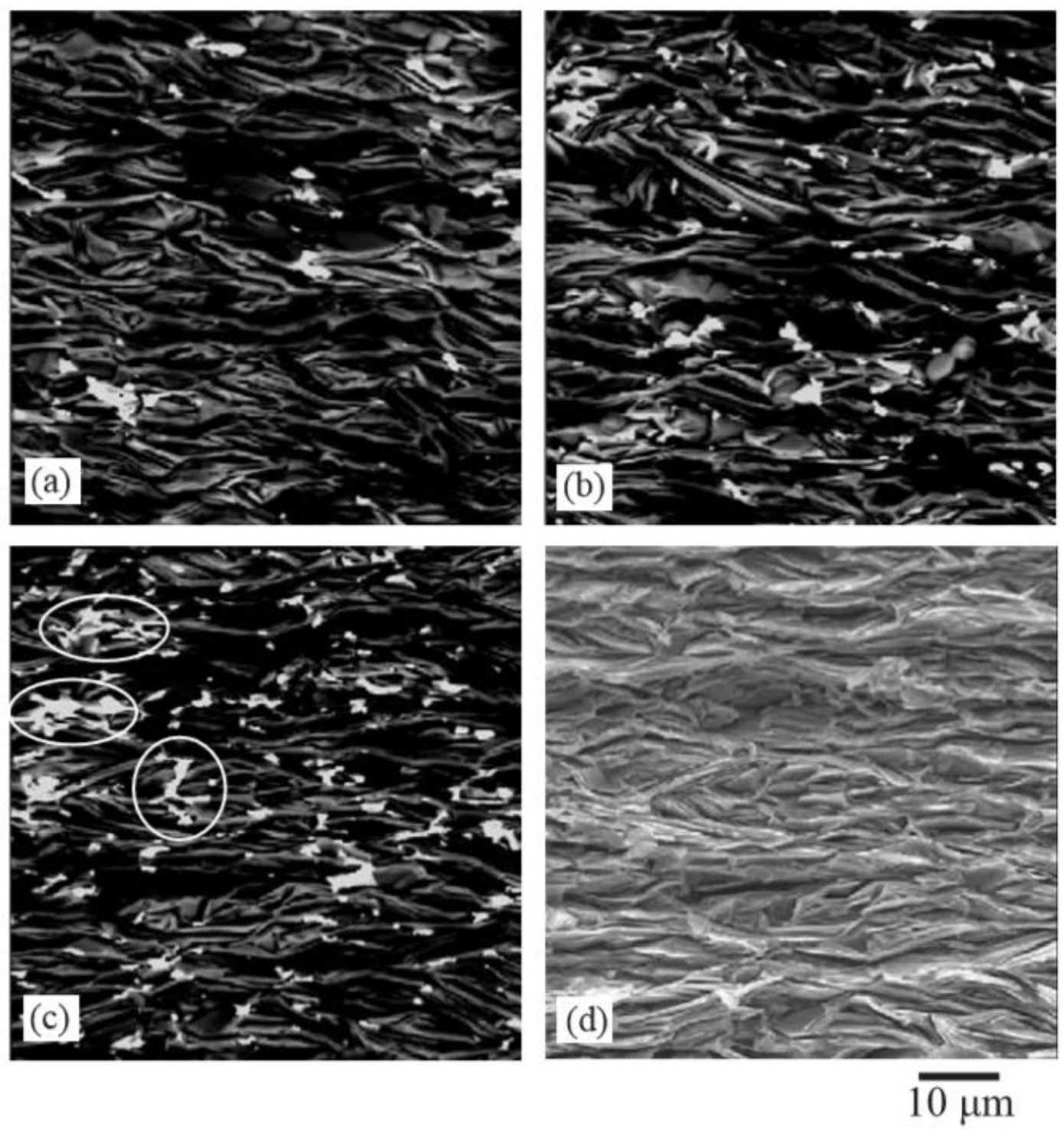

Figure 9. BSE images of fractured sections of CCO/Ag composites (a) $5 w t \%$, (b) $10 w t \%$, (c) $20 w t \%$ and SEM (d) $20 w t \%$. Ag metallic phase is circled in (c) .[47]

The size of nanoinclusions is critical in reducing the thermal conductivity, the nanoinclusions size should be comparable to the phonon mean free path to be more effective; also, as the size of the nanoinclusions decrease, the thermal conductivity decreases linearly [53]. 
On this nanoinclusions addition approach the lowest Ag sizes reported are of around $350 \mathrm{~nm}$ [54].in Figure 10 the schematic illustration of the strategies for microstructure improvement of the CCO polycrystalline materials is shown.

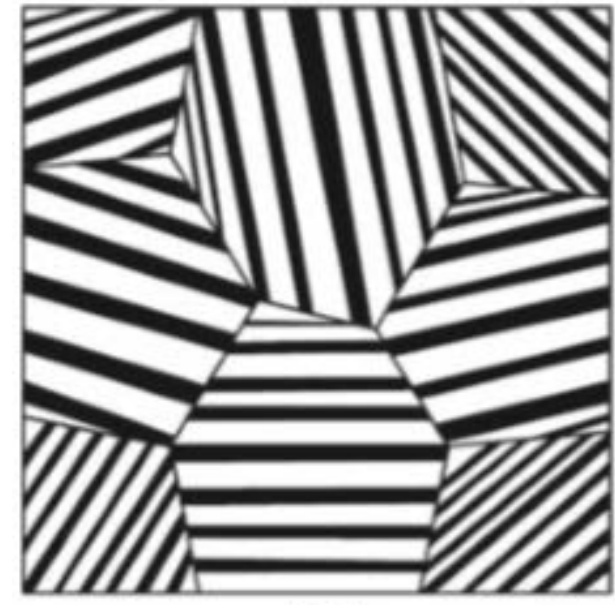

(a)

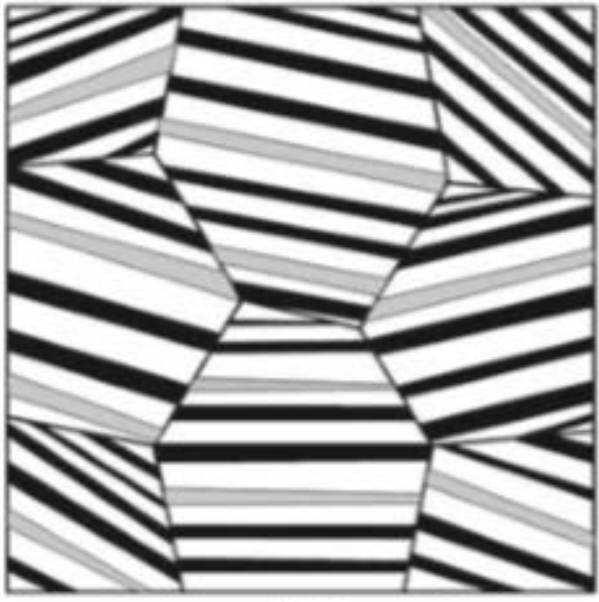

(c)

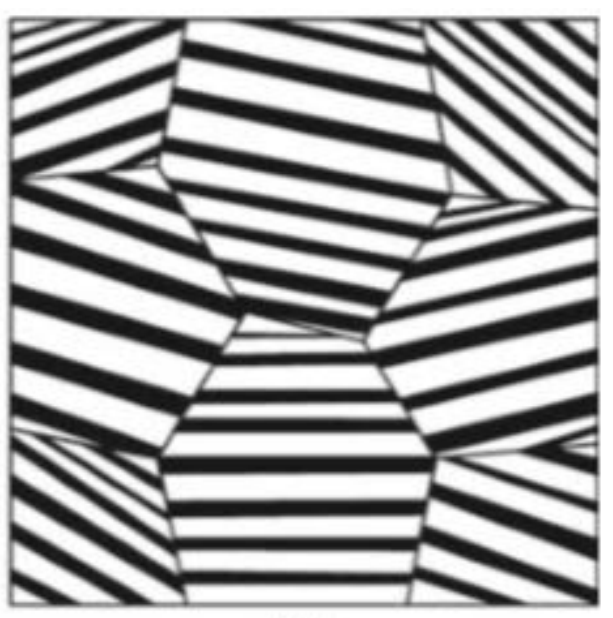

(b)

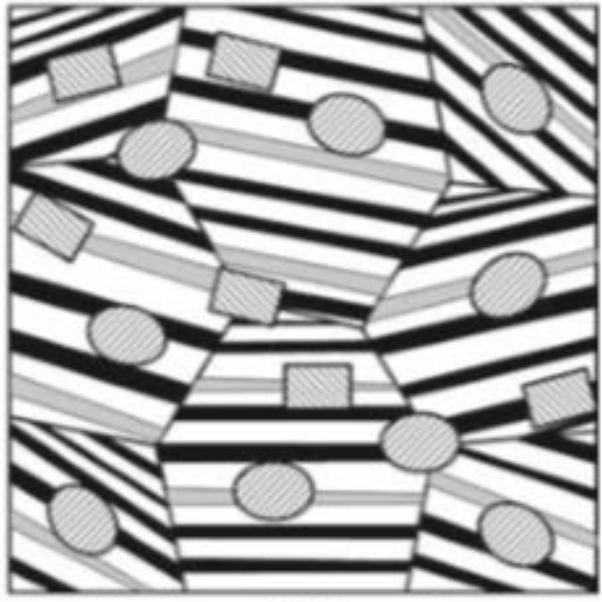

(d)

Figure 10. Strategies for improvement of TE performance of polycrystalline CCO. (a) lamellar nanostructures, (b) preferentially oriented lamellar nanostructures, (c) oriented lamellar nanostructures with heavy element doping and (d) preferentially oriented lamellar nanostructures with heavy element doping and nano-sized inclusions.[46]

However the efficiency of these polycrystalline materials seems to be bellow the best performance (single crystal case), further study and improvement of thermoelectric materials should be carried out. 
In this research, impurity doping and addition of nanoparticles is performed to study the transport properties and microstructure of $\mathrm{Ca}_{3} \mathrm{CO}_{4} \mathrm{O}_{9}$ (CCO) system.

\subsection{Research Goals and Objectives}

The goal of this research is to improve the thermoelectric performance of the $\mathrm{Ca}_{3} \mathrm{Co}_{4} \mathrm{O}_{9}$ polycrystalline systems through engineering the nanostructure and chemistry.

In detail, the objectives of the present research are the following:

To synthesize crystal textured Bi-doped CCO ceramic samples.

To study the effect of Bi doping on the thermoelectric performance of the CCO system.

To investigate the effect of Bi doping on the microstructure of polycrystalline CCO samples.

To reveal the microstructure origin of the changes induced by $\mathrm{Bi}$ doping on the thermoelectric performance of the CCO system.

To synthesize the CCO polycrystalline samples with Au nanoinclusions and study the effect of nanoinclusions on the nanostructure and TE properties of the samples. 


\section{CHAPTER 2. Principles for the Materials Synthesis of Thermoelectric Materials and Thermoelectric Performance Measurement}

\section{First Part: Materials Synthesis}

\subsection{Synthesis Methods for Ceramics: Powder Samples}

There are many methods for the synthesis of TE materials; they are divided in two main categories: physical and chemical routes. For the specific case of CCO manufacturing, the most commonly used are Solid State Reaction (Physical) and Sol-Gel Synthesis (Chemical). Both of them allow well controlled composition, however the sol-gel route as a bottom up technique permits to have more control on the size and morphology of the materials.[55]

\subsubsection{Solid State Reaction}

In the solid state reaction process precursor solid compounds are mixed in the appropriate stoichiometric ratios, subsequent pressure and temperature exposures induce decomposition of the reagents and re-crystallization of the materials in the desired compound. Generally large grain size materials are obtained from this processing route since prolonged high temperature annealing processes induce grain growth in the compound[55].

\subsubsection{Sol-Gel Route}

The sol-gel method consist of dissolution of the metal ion precursors in a water based solution, including some gel forming agents and $\mathrm{PH}$ adjusters to allow for metal ions to form complexes; then the solution is heated under stirring and a gel is formed. Removal of the organic compounds is achieved by calcination of the gel; in this step, the material crystallizes to the target compound.

Compared to the solid state reaction method, the sol-gel route is better because it allows much more control over size and shape of the crystallized compound and gives more cation stoichiometry. For the sol-gel route no intermediate grinding procedures are needed; which are required for the solid state reaction processing to overcome the diffusion problems. 
Additional advantages of the sol-gel route among other techniques are low temperature manipulation temperatures, stability and high homogeneity of the obtained material.

\subsubsection{Hydrothermal Deposition Method}

The hydrothermal processing is a synthesis route that involves high pressure and temperature within an aqueous media. It comes from the nature's process to form different compounds in which these conditions are met along with large periods of time. The hydrothermal route allows obtaining materials that were thought to be found only in nature such as diamond, quartz and other spinel systems.[56]

Growth of single big crystals as well as nanoparticles can be done by changing the hydrothermal synthesis parameters such as time, temperature, pressure, solvent and $\mathrm{PH}$ of the precursor solution. In the process, a solution of the compound is formed and placed in a vessel, and then it is submitted to a temperature - pressure program where the desired compound crystallizes.

\subsection{Sample Densification (Pellets Preparation)}

After the compound crystallization to powders, formation and densification of samples is required. The powder material is placed into a die where pressure is applied to form the samples. Heat can also be applied to the system, in this case the processing is called hot pressing - HP; in absence of heat it is called cold pressing - CP. The schematic setting for cold/hot pressing is shown in Figure 11. 


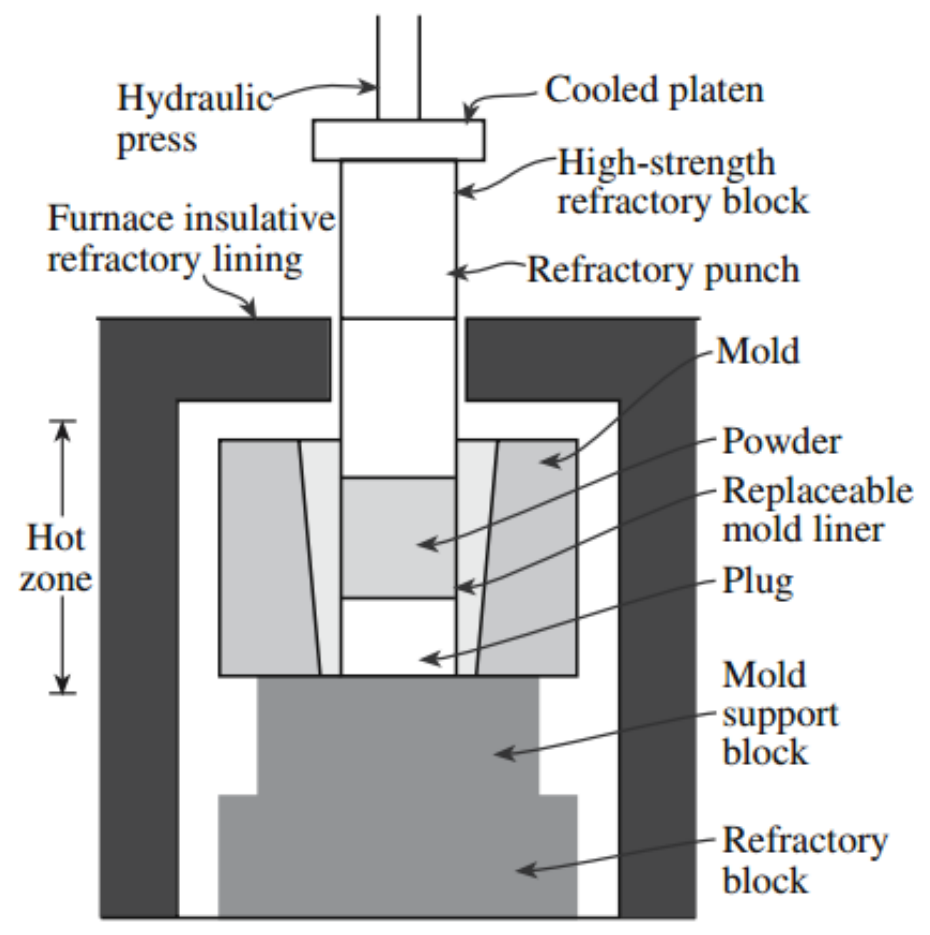

Figure 11. Schematic setting for hot (cold in absence of heat) pressing. [57].

After sample formation, annealing is required. In this step the formed samples are submitted to temperatures near their melting temperature for long (9-24 hours) periods of time to induce grain growth and subsequent increase of the density. There is risk of cracking and void formation if the temperature program is not appropriate.

An alternative method to densification for this kind of samples is spark plasma sintering. In this method the sample is pressed in a die to be formed and is followed by a DC electrical current or a pulse of $A C$ current to sinter the compound instantaneously. Some advantages of this technique among HP and CP are high through put, temperature gradient minimization at the interior of the sample, preservation of nano-sized features and higher densification of the samples up to 95\%.[55] The schematic setting of this technique is shown in Figure 12. 


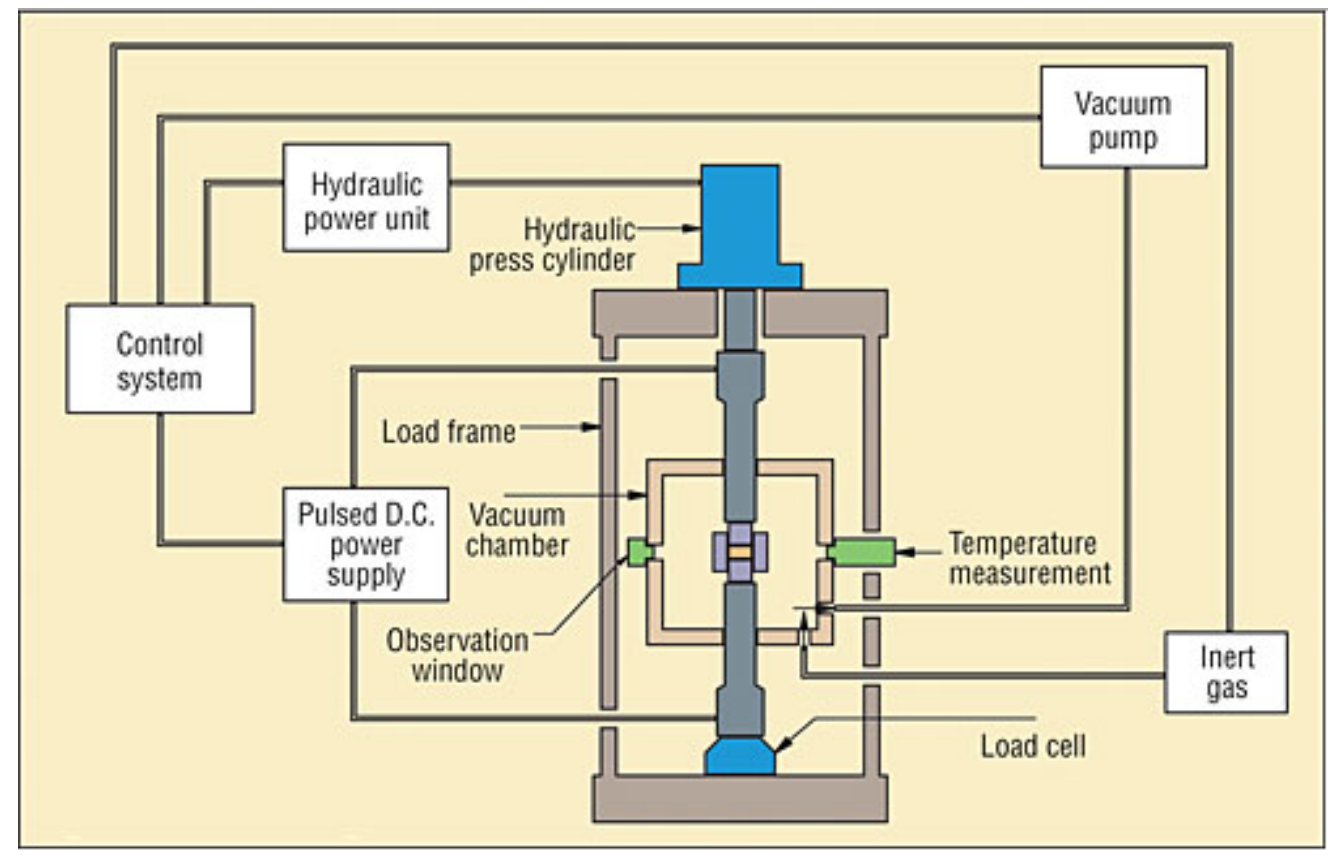

Figure 12. Schematic setting of spark plasma sintering. [58].

Comparative studies of the differences in mechanical properties and transport properties obtained for these different forming-sintering processes have been widely studied [17-21].

\section{Second Part: Thermoelectric Performance Measurement}

\subsection{Methods for Measuring Transport Properties: Seebeck Coefficient, Electrical Conductivity and Thermal Conductivity.}

The transport properties are measured directly from the material samples, the figure of merit representing the thermoelectric performance is then calculated from Equation 1.

On the measuring process great care should be taken to have realistic estimation on the materials behavior, among the main considerations are measuring properties from the same sample when possible or at least from a sample with the exact processing conditions, measuring the properties from samples from the same batch and fabrication time to eliminate possible sample deterioration effects, 
and taking measurements of the same directions on the samples since transport properties for the layered TE materials are different in different directions.

The methods described below are the most accepted for measuring the transport properties.

\subsubsection{Seebeck Coefficient and Electrical Conductivity}

The Seebeck coefficient and electrical resistivity can be measured simultaneously using the Four Probe method, this method consist of two contacts inducing a current $I$ and another two contacts measuring the voltage $V$. See Figure 13 . The electrical conductivity can be estimated directly; for the Seebeck coefficient a temperature gradient is created and the generated voltage gradient is measured; corrections should be performed to compensate for error due to the materials with which the temperature sensors are fabricated. The expressions for Seebeck coefficient $\alpha$ and electrical conductivity $\sigma$ are:

$$
\begin{gathered}
\alpha=\frac{\Delta V}{\Delta T} \\
\sigma=\frac{1}{\rho} \\
\rho=\frac{\Delta V}{\Delta I} \frac{A}{L}
\end{gathered}
$$

Where $A$ is the cross sectional area and $L$ is the distance between the voltage measuring contacts. 


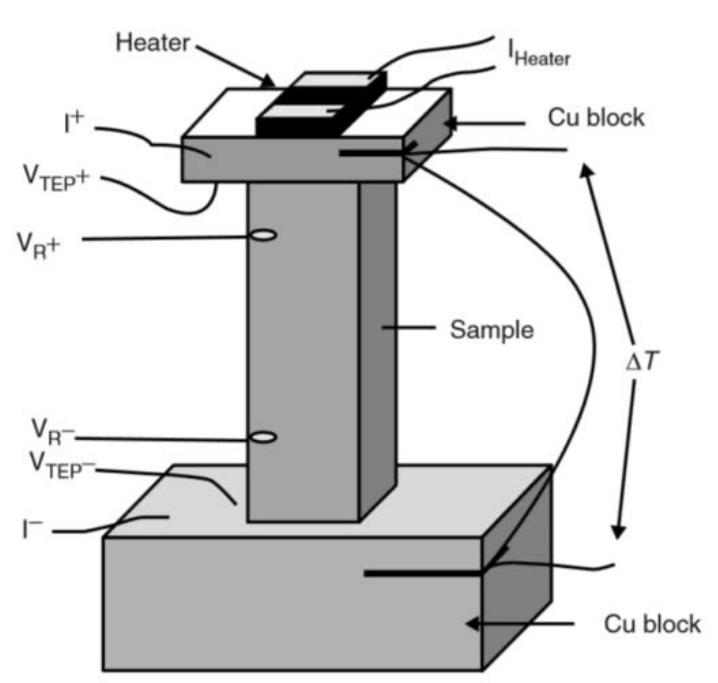

Figure 13. Four probe setting.[4]

\subsubsection{Thermal Conductivity}

For the thermal conductivity, the Laser Flash method is used. In this method the sample is flashed with a laser for a period shorter than $1 \mathrm{~ms}$ and an IR detector monitoring the other side of the sample collects the signal to calculate for the thermal diffusivity and specific heat. In Figure 14 the illustration of the principle is shown.

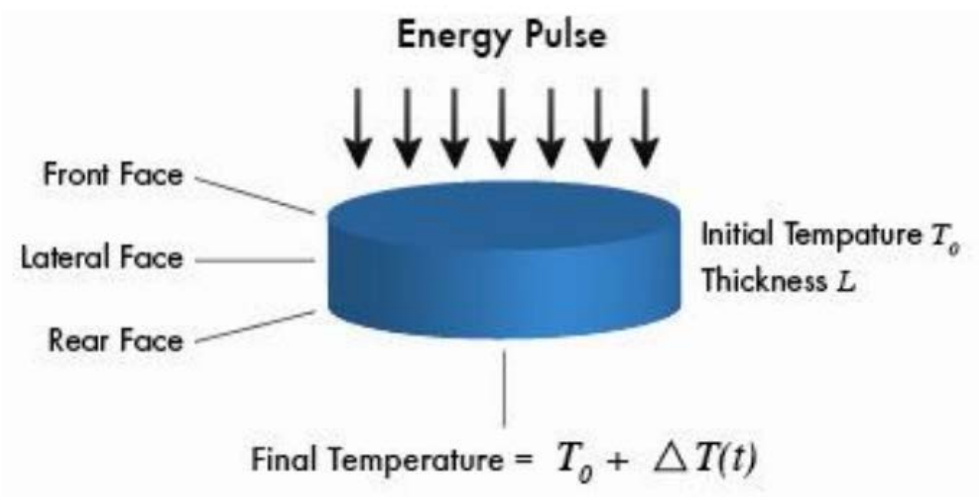

Figure 14. Schematic Laser Flash method.[59]

The expression for the thermal conductivity is:

$$
\lambda=d \mu C_{p}
$$

Where $d$ is the thermal diffusivity, $\lambda$ is the thermal conductivity, $\mu$ is the density of the sample and Cp is the specific heat. 


\section{Third Part: Nanostructure and Chemistry Characterization using Scanning TEM (STEM)}

\subsection{Scanning Transmission Electron Microscope STEM}

Scanning transmission electron microscopy allows characterization of material features down to single atom levels. A high energy electron beam is focused and passed through an electron transparent sample. The electrons encountering the sample will be scattered by the atoms, later the scattered electrons and ionized atoms signal is collected and recorded. As the electron beam scans the sample, maps of selected areas with chemical composition and chemical bonding information are obtained. Elastically scattered electrons are collected with the annular dark field detector - ADF giving mass contrast information of the elements in the sample. The inelastically scattered electrons are collected with a spectrometer and analyzed making use of the electron energy loss spectroscopy - EELS technique giving compositional and atomic bonding information [60].

The refined analysis of the STEM is possible due to the advances in the aberration correctors technology which allow for probe sizes in the order of sub-angstroms and for much more efficient collection times [60].These features make possible detailed to obtain information of specific regions such as grain boundaries and interfaces between different materials.

The schematic setting of the STEM machine is shown in Figure 15. 


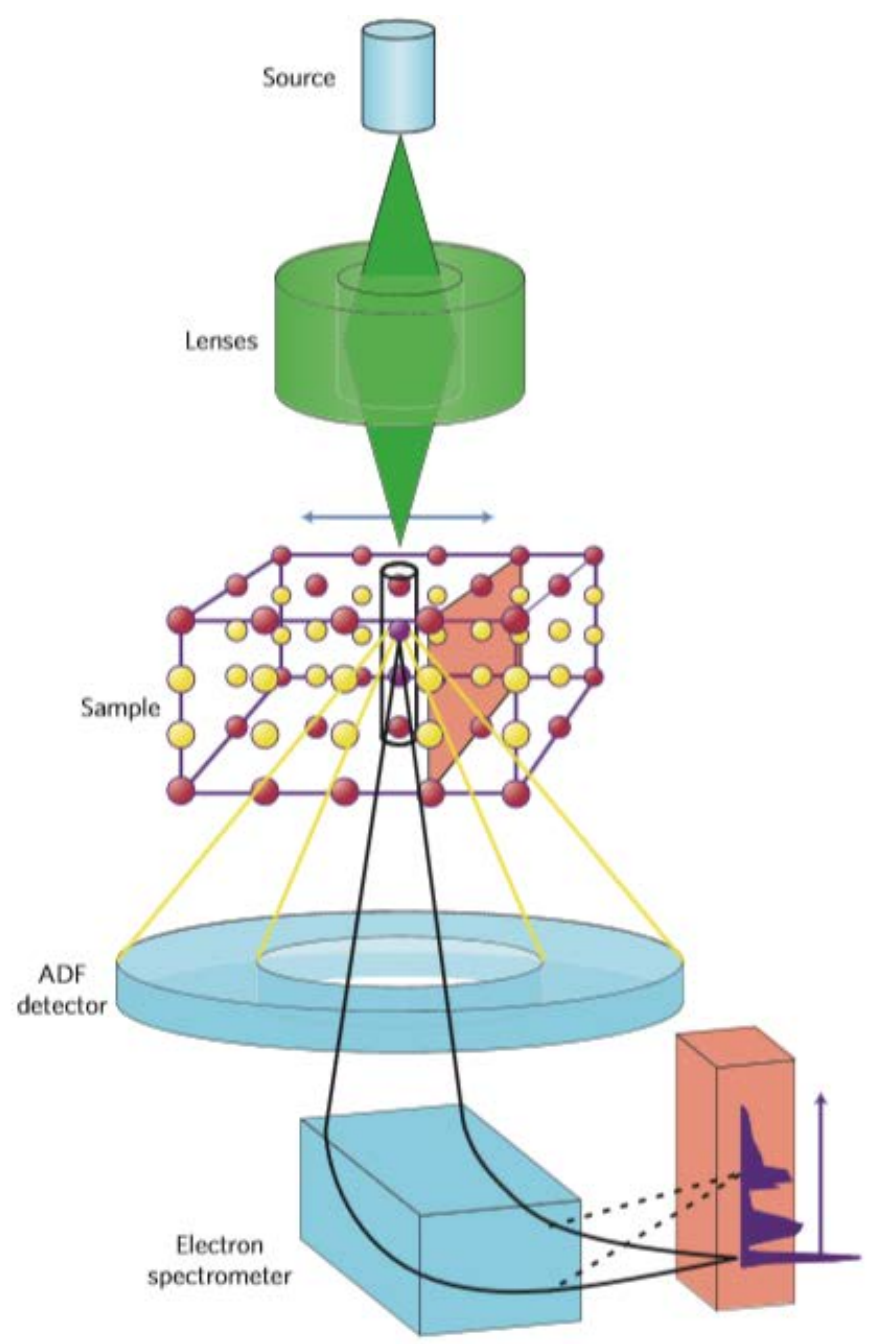

Figure 15. Schematic configuration and major elements of a scanning transmission electron microscope STEM.[60] 


\section{CHAPTER 3. Effect of Bi Doping on the Ca-site $\left(\mathrm{Ca}_{3-\mathrm{x}} \mathrm{Bi}_{\mathrm{x}} \mathrm{Co}_{4} \mathrm{O}_{9}\right)$ on the Thermoelectric Performance and Nanostructure}

\subsection{Methodology}

In this research the properties of $\mathrm{CCO}$ are studied by doping with $\mathrm{Bi}$ and integration of $\mathrm{Au}$ nanoinclusions to the systems. Characterization of the obtained materials and their microstructure has been done though XRD, SEM, TEM, EDS; the transport properties were measured using the four probe method for the Seebeck coefficient and electrical conductivity, and using Laser Shot method for the thermal conductivity.

\subsubsection{Sol-gel Synthesis of the powders}

Precursor raw materials were mixed in deionized water in stoichiometric ratios, ethylene glycol and polyethylene glycol were used to polymerase the solution, nitric acid was also added to induce nitrate salts decomposition and facilitate new compound formation. A summary table of the precursor materials is shown in Table 1.The solution was mechanically stirred to $200 \mathrm{rpm}$ at $80^{\circ} \mathrm{C}$ during 3 hours to form the gel. It was then placed in small crucibles and set to ashes by firing it to $500^{\circ} \mathrm{C}$ during 2 hours. The resulting ashes were collected and grinded for 20 minutes and a fine powder was obtained. This was submitted to the tube furnace for calcination at $650^{\circ} \mathrm{C}$ during 4 hours and oxygen flow to have stoichiometric concentration of the later. The obtained powder was black and was characterized as $\mathrm{Ca}_{3} \mathrm{Co}_{4} \mathrm{O}_{9}-\mathrm{CCO}$ using XRD. In the subsequent chapters XRD detailed information is presented for each of the systems studied.

Table 1. Precursor constituents of Sol-gel CCO and CCO-doped/nanoinclusions added systems.

\begin{tabular}{|c|c|}
\hline Reagents & Formula \\
\hline Deionized Water & $\mathrm{H}_{2} \mathrm{O}$ \\
\hline Polyethylene Glycol & $\left(\mathrm{C}_{2} \mathrm{H}_{4} \mathrm{O}_{6}\right)_{\mathrm{n}}-\mathrm{C}_{2} \mathrm{H}_{6} \mathrm{O}_{2}$ \\
\hline Ethylene Glycol & $\mathrm{C}_{2} \mathrm{H}_{6} \mathrm{O}_{2}$ \\
\hline Citric Acid & $\mathrm{C}_{6} \mathrm{H}_{8} \mathrm{O}_{7}$ \\
\hline Nitric Acid & $\mathrm{HNO}_{3}$ \\
\hline Urea & $\left(\mathrm{NH}_{2}\right)_{2} \mathrm{CO}$ \\
\hline Calcium Nitrate Tetrahydrate & $\mathrm{Ca}\left(\mathrm{NO}_{3}\right)_{2}-4 \mathrm{H}_{2} \mathrm{O}$ \\
\hline Cobalt Nitrate Hexahydrate & $\mathrm{Co}\left(\mathrm{NO}_{3}\right)_{2}-6 \mathrm{H}_{2} \mathrm{O}$ \\
\hline
\end{tabular}


CHAPTER 3. EFFECT OF Bi DOPING ON THE Ca-SITE $\left(\mathrm{Ca}_{3-x} \mathrm{Bi}_{x} \mathrm{Co}_{4} \mathrm{O}_{9}\right)$ ON THE THERMOELECTRIC PERFORMANCE AND NANOSTRUCTURE

\begin{tabular}{ll}
\multicolumn{1}{c}{ Reagents } & \multicolumn{1}{c}{ Formula } \\
\hline Bismuth Nitrate Pentahydrate & $\mathrm{Bi}\left(\mathrm{NO}_{3}\right)_{3}-5 \mathrm{H}_{2} \mathrm{O}$ \\
Hydro Auric Chloride Acid & $\mathrm{HAuCl}_{4}-3 \mathrm{H}_{2} \mathrm{O}$ \\
\hline
\end{tabular}

\subsubsection{Pressing and Sintering}

The CCO powder was made into pellet form of $9 \mathrm{~mm}$ diameter. Samples were pressed at $1 \mathrm{GPa}$ and $150^{\circ} \mathrm{C}$ during 40 minutes. The density of the obtained discs was measured. The green pellets were sintered at $920^{\circ} \mathrm{C}$ during 9 hours in oxygen flow and density measurements were carried out once more. The final density values for each set of samples of the systems studied is presented in the subsequent chapters.

\subsubsection{Data Acquisition}

To measure the transport properties two main groups of information were taken. The Seebeck coefficients and electrical conductivities were obtained by the four probe method using a LINSEIS LSR-3 Seebeck machine. The samples were cut to approximate dimensions of $3 \times 3 \times 8 \mathrm{~mm}$ for his measurement; for the second group of data, the thermal properties were measured by the laser shot method using a LINSEIS LFA 1000 laser flash machine. The dimensions for the samples for thermal measurements are $2 \times 9 \times 12 \mathrm{~mm}$.

For further understanding of the studied systems and their microstructure, characterization and imaging was performed. XRD measurements were taken with a PANalyticalXPert-Pro XRD machine using Cu Kalpha radiation, $45 \mathrm{kV}$ tension and $40 \mathrm{~mA}$ current; SEM images were obtained with a JEOL JSM-7600F scanning electron microscope using SEI mode, acceleration voltage of $15 \mathrm{kV}$ and working distance of 6 $\mathrm{mm}$. TEM imaging and EDS compositional information were obtained with a JEOL JEM-2100 transmission electron microscope using $200 \mathrm{kV}$. The Scanning TEM (STEM) imaging and EDS analysis was carried out on the JEM-2100F with Cs correctors operated at 200KV.

\subsection{Baseline pure CCO}

A baseline set of samples was also fabricated for comparison purposes, the composition is $\mathrm{Ca}_{3} \mathrm{Co}_{4} \mathrm{O}_{9}$. All the manufacturing conditions are kept identical.

Images of the precursor powders of baseline CCO are shown in Figure 16 and Figure 17. The grain size varies from approximately $15 \mathrm{~nm}$ to $500 \mathrm{~nm}$. All grains show platelet shape, the smaller sized grains are rather circular, whereas the larger grains look randomly shaped. 


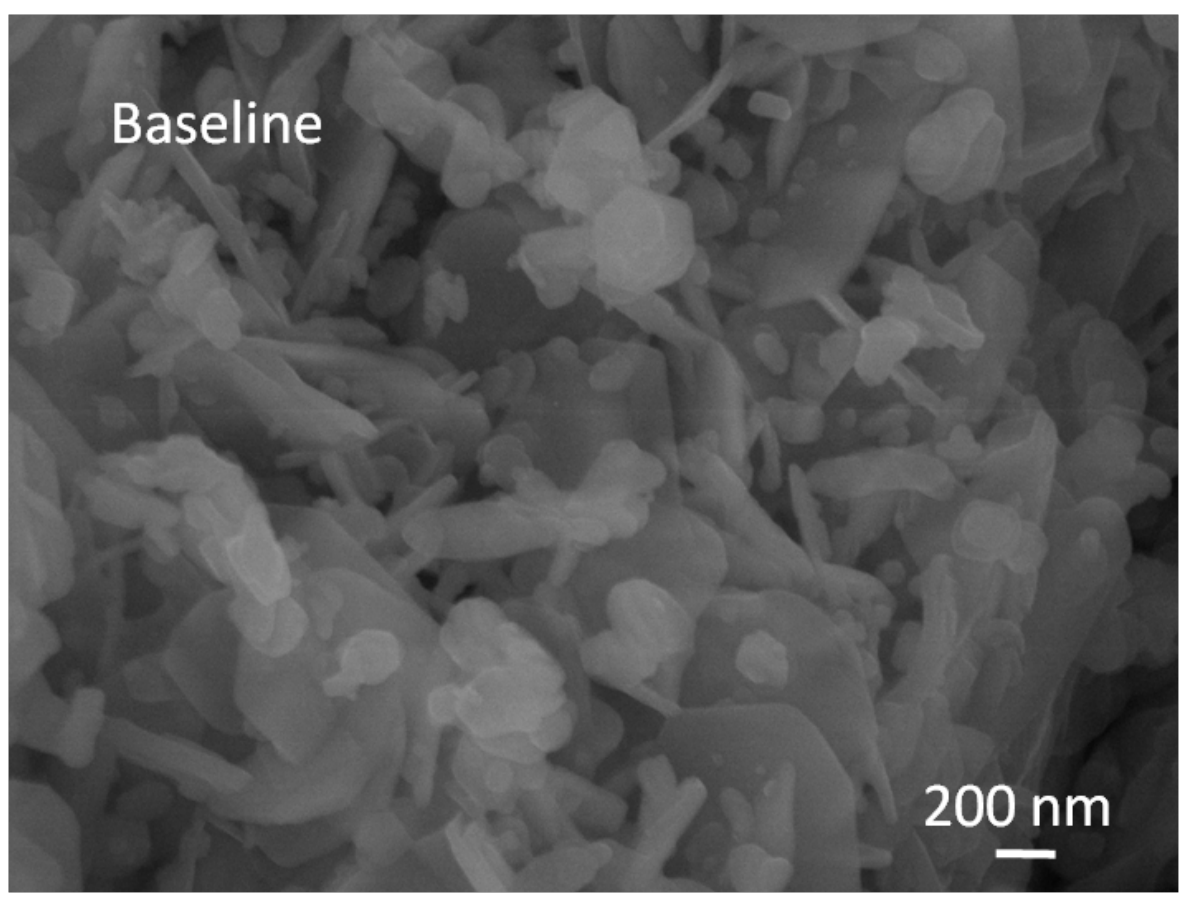

Figure 16. SEM image of CCO baseline powders after calcination at $650^{\circ} \mathrm{C}$ for 4 hours.

The material is of crystalline nature as observed through TEM analysis. Notice that there is some texturing of the surface of the grains, see TEM images in Figure 17. This feature is also observed for the Bi doped systems as shown in Bi Doping section.

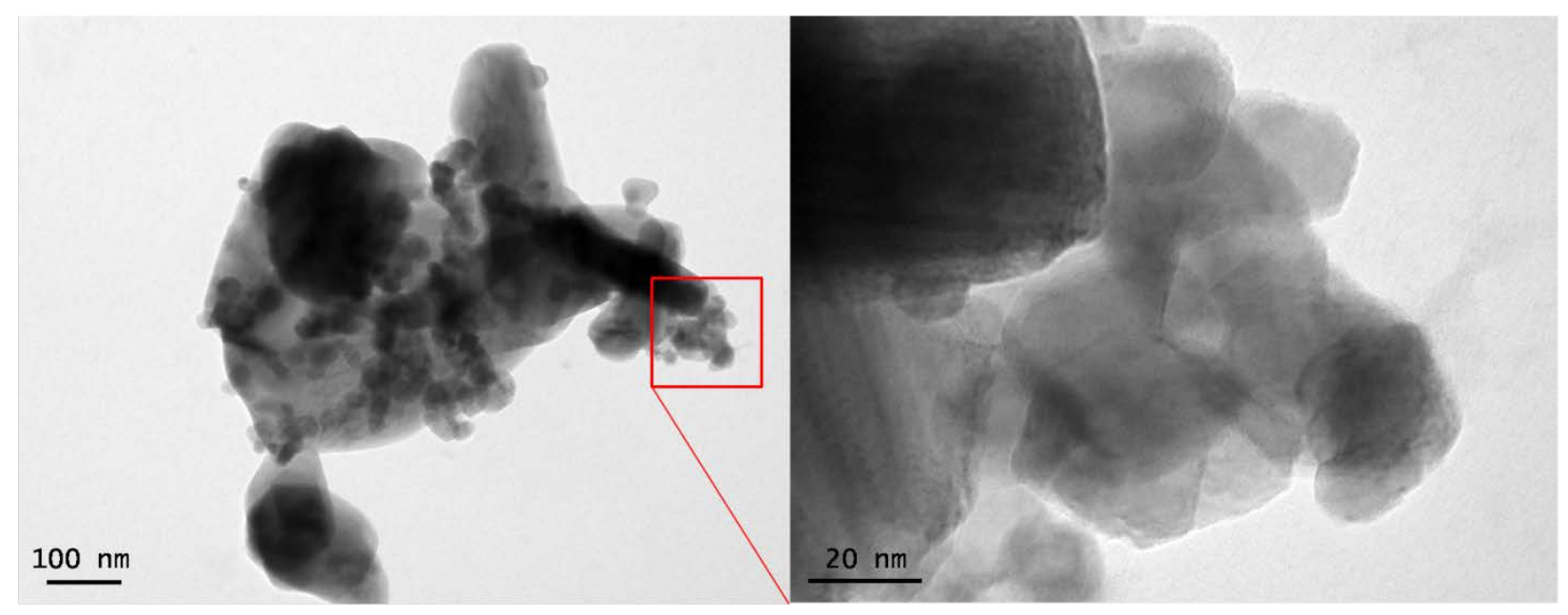

Figure 17. TEM images of the baseline $\mathrm{CCO}$ powders after calcination at $650^{\circ} \mathrm{C}$ for 4 hours. 
CHAPTER 3. EFFECT OF Bi DOPING ON THE Ca-SITE $\left(\mathrm{Ca}_{3-x} \mathrm{Bi}_{x} \mathrm{Co}_{4} \mathrm{O}_{9}\right)$ ON THE THERMOELECTRIC PERFORMANCE AND NANOSTRUCTURE

Compositional analyses of the samples using EDS confirm the atomic ratio to be $\mathrm{Ca}_{3} \mathrm{Co}_{4} \mathrm{O}_{9}$. Additionally cobalt oxide with low calcium content (around 1\%) was found to form the smaller crystals of the compound. 


\subsection{Calcium Site Doping $\left(\mathrm{Ca}_{(3-\mathrm{x})} \mathrm{Bi}_{\mathrm{x}} \mathrm{Co}_{4} \mathrm{O}_{9}\right)$}

The addition of heavy atoms to thermoelectric materials in doping levels is expected to reduce the thermal conductivity of the compounds [42]. Traditionally, $\mathrm{Bi}$ is used as thermoelectric material in intermetallic compounds with Te; such compounds are the most widely used for thermoelectric applications at low temperature. However the high toxicity of Te is a major drawback of these materials.

The addition of $\mathrm{Bi}$ in the $\mathrm{CCO}$ system is thought to reduce de thermal conductivity and to increase the density of the compound.

\subsubsection{Synthesis and Sample Conformation}

Substitution of the Calcium site in the $\mathrm{CCO}$ was introduced by doping in concentrations of $\mathrm{x}=0.1,0.2,0.3$ and 0.4. The composition formula is $\mathrm{Ca}_{(3-x)} \mathrm{Bi}_{x} \mathrm{Co}_{4} \mathrm{O}_{9}$. The powder samples were synthesized though the sol-gel method to their final composition. Then pressed into pellets and sintered as described in 3.1.2 Pressing and Sintering and finally cut into appropriate dimensions for data acquisition.

Images of the precursor powders are shown in Figure 18. Similar powder size is observed for all $\mathrm{Bi}$ concentration doped systems and is also comparable to that for the baseline powder. The average size for the smaller grains in the Bi 0.3 doped precursor powders is $\sim 20 \mathrm{~nm}$ and for the larger grains is $\sim 500$ $\mathrm{nm}$ as seen in Figure 19. The powder platelet like shape is also similar to the one observed for the baseline powders; there is however apparent augmenting presence of smaller grains of cobalt oxide as $\mathrm{Bi}$ addition increases. 

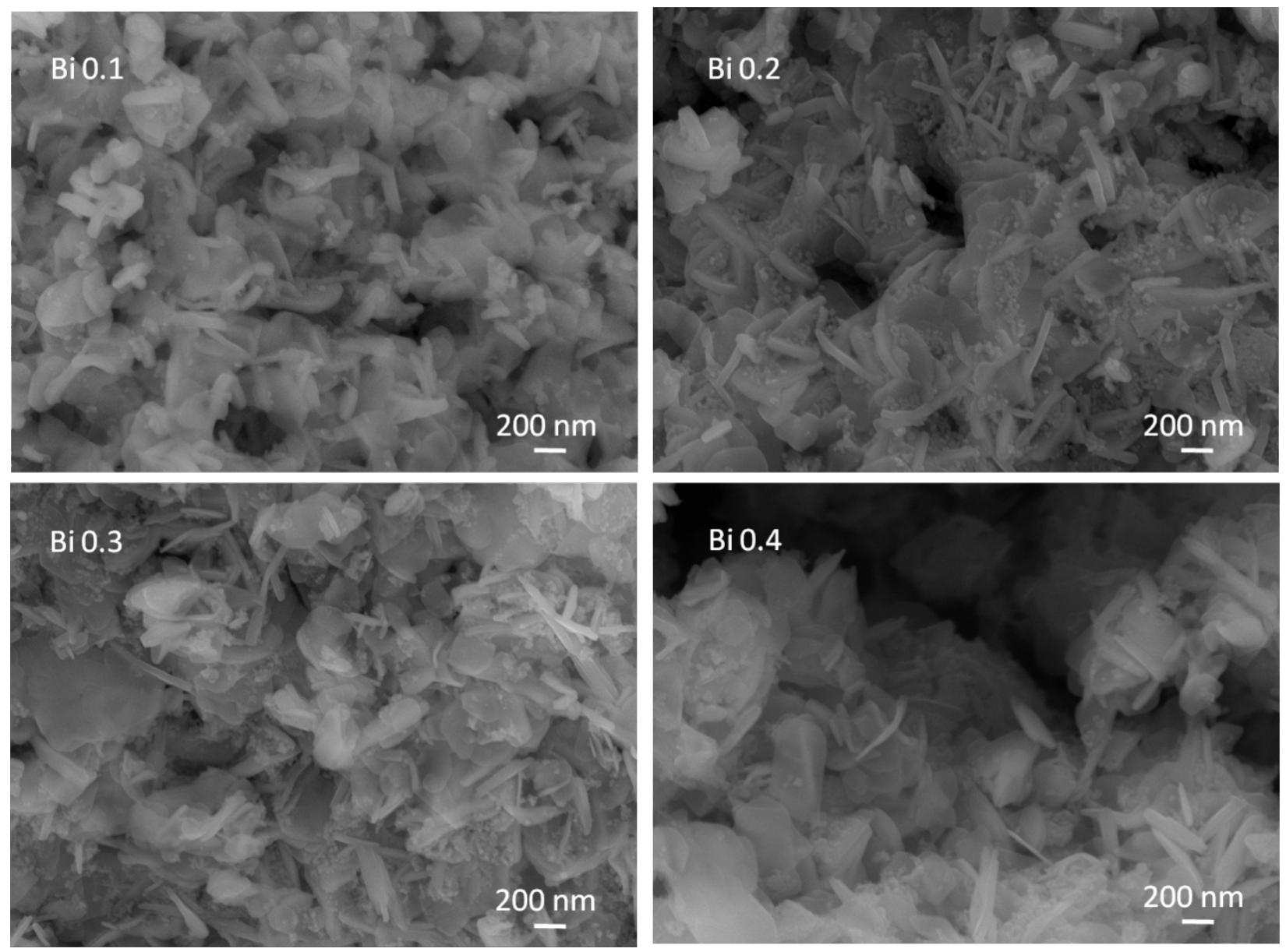

Figure 18. SEM images of Bi doped CCO powders after calcination at $650^{\circ} \mathrm{C}$ for 4 hours.

TEM images of the Bi 0.3 doped powders are shown in Figure 19. Fine texture of the surface of the grains is observed as pointed out for the baseline, this appears to be a common characteristic of the precursor powders; insets show small spherical features of $\sim 3 \mathrm{~nm}$ to $\sim 4 \mathrm{~nm}$ on the surface, similar to those present in the baseline powder. All grains were found to be of crystalline nature. 


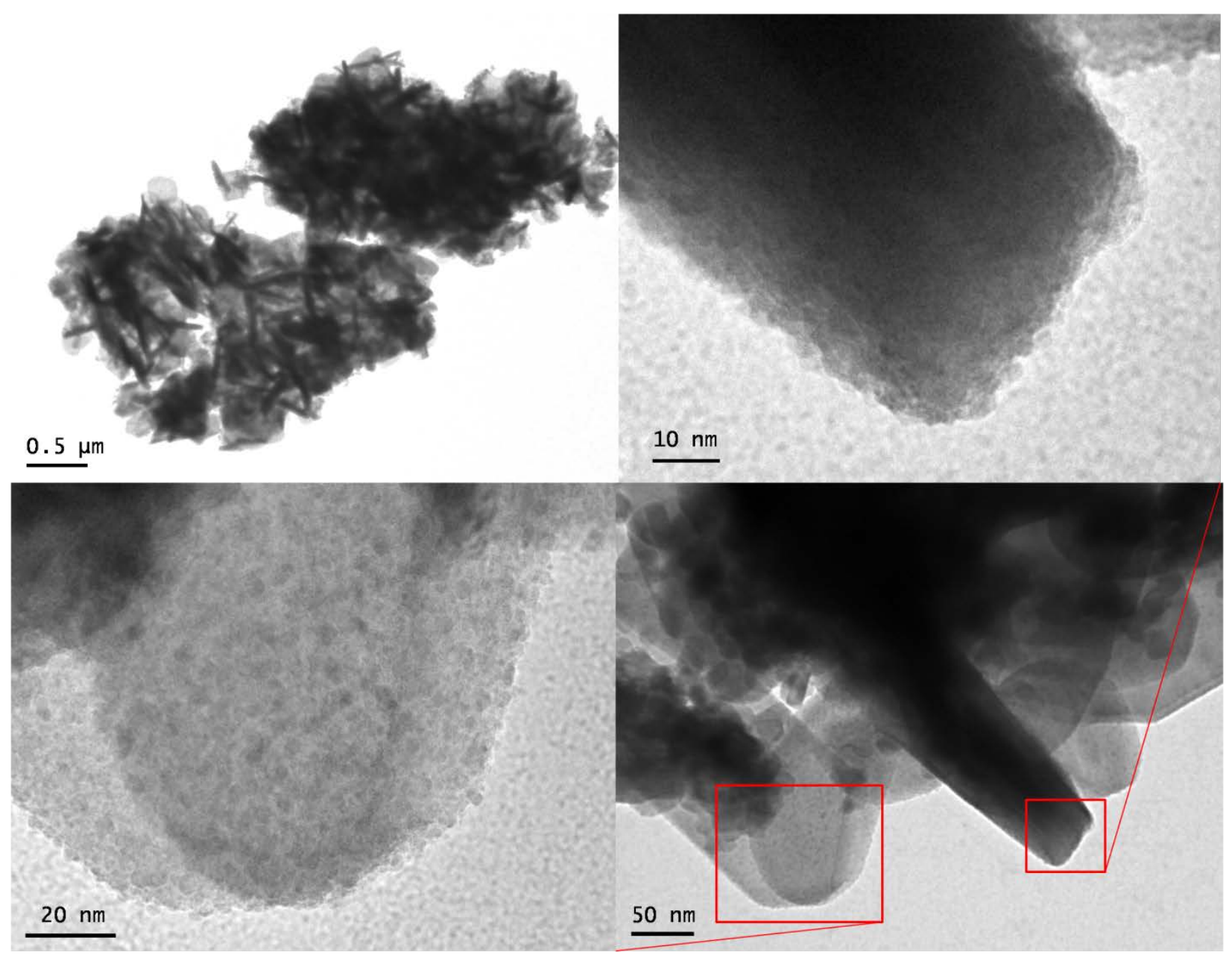

Figure 19. TEM images of Bi 0.3 doped powders after calcination at $650^{\circ} \mathrm{C}$ for 4 hours.

The EDS analysis of the composition of the powders in Figure 19 showed a rather uniform presence of $\mathrm{Bi}$ in the compound of 0.3 atomic \%. Ca and Co content are also consistent with $\mathrm{CCO}$ atomic ratios.

\subsubsection{Density of the Pellets}

The pure CCO single crystal exhibits a density of $4.47 \mathrm{~g} / \mathrm{cm}^{3}$, slightly lower than the theoretical value $4.68 \mathrm{~g} / \mathrm{cm}^{3}$.[13] In Table 2, the measured density values for the Bi set of samples is summarized. From the density values it is clear that the addition of $\mathrm{Bi}$ as dopant increases the density of the compound 
CHAPTER 3. EFFECT OF Bi DOPING ON THE Ca-SITE $\left(\mathrm{Ca}_{3-x} \mathrm{Bi}_{x} \mathrm{Co}_{4} \mathrm{O}_{9}\right)$ ON THE THERMOELECTRIC PERFORMANCE AND NANOSTRUCTURE

reaching relative densities of $95 \%$ for $\mathrm{Bi} 0.3$ and $103 \%$ for $\mathrm{Bi} 0.4$. The later values are significantly larger than those for the baseline with relative density of $85 \%$.

Table 2. Density of CCO systems, baseline and Bi doped series.

\begin{tabular}{lccc}
\multicolumn{2}{c}{ Sample } & Density $\left(\mathrm{g} / \mathrm{cm}^{3}\right)$ & Relative Density (\%) \\
\hline Single crystal CCO & & 4.68 & 100 \\
Baseline 1 GPa & $\mathbf{X}$ & 3.97 & 85 \\
& 0.1 & & \\
& 0.2 & 4.05 & 87 \\
$\mathrm{Bi}$ & 0.3 & 4.10 & 88 \\
& 0.4 & 4.46 & 95 \\
& & 4.83 & 103 \\
\hline
\end{tabular}

\subsubsection{XRD Measurements}

XRD of the compound series shows shifting of the peak position with the introduction of $\mathrm{Bi}$ to the CCO system, the characteristic $\mathrm{Ca}_{3} \mathrm{Co}_{4} \mathrm{O}_{9}$ corresponding peaks [13] were present for all samples regardless of the $\mathrm{Bi}$ contain indicating that doping of the system occurred. Table 3 presents the XRD peak summary of the synthesized CCO - Bi doped powders.

Table 3. Powder X-Ray diffraction indexed peaks of CCO Baseline and Bi $x=0.1$ to 0.4 doped systems.

\begin{tabular}{|c|c|c|c|}
\hline hkl & Sample & 2-Theta position $\left({ }^{\circ}\right)$ & d-spacing $(\AA)$ \\
\hline \multirow{5}{*}{002} & Baseline & 16.5251 & 5.3646 \\
\hline & $x=0.1$ & 16.4680 & 5.3830 \\
\hline & $x=0.2$ & 16.4124 & 5.4011 \\
\hline & $x=0.3$ & 16.4166 & 5.3830 \\
\hline & $x=0.4$ & 16.3891 & 5.4088 \\
\hline \multirow{5}{*}{003} & Baseline & $*$ & $*$ \\
\hline & $x=0.1$ & $*$ & $*$ \\
\hline & $x=0.2$ & $*$ & * \\
\hline & $x=0.3$ & $*$ & $*$ \\
\hline & $x=0.4$ & 24.7612 & 3.5957 \\
\hline \multirow{5}{*}{$11-2$ S1 } & Baseline & $*$ & $*$ \\
\hline & $x=0.1$ & $*$ & $*$ \\
\hline & $x=0.2$ & $*$ & $*$ \\
\hline & $x=0.3$ & 30.2065 & 2.9588 \\
\hline & $x=0.4$ & 30.1899 & 2.9604 \\
\hline
\end{tabular}


CHAPTER 3. EFFECT OF Bi DOPING ON THE Ca-SITE $\left(\mathrm{Ca}_{3-x} \mathrm{Bi}_{x} \mathrm{Co}_{4} \mathrm{O}_{9}\right)$ ON THE THERMOELECTRIC PERFORMANCE AND NANOSTRUCTURE

\begin{tabular}{|c|c|c|c|}
\hline hkl & Sample & 2-Theta position $\left({ }^{\circ}\right)$ & d-spacing ( $\AA$ ) \\
\hline \multirow{5}{*}{112 S1 } & Baseline & $*$ & $*$ \\
\hline & $x=0.1$ & $*$ & $*$ \\
\hline & $x=0.2$ & $*$ & $*$ \\
\hline & $x=0.3$ & 33.1492 & 2.7026 \\
\hline & $x=0.4$ & 33.1399 & 2.7033 \\
\hline \multirow{5}{*}{004} & Baseline & 33.4295 & 2.6805 \\
\hline & $x=0.1$ & 33.2687 & 2.6931 \\
\hline & $x=0.2$ & 33.2311 & 2.6961 \\
\hline & $x=0.3$ & $*$ & $*$ \\
\hline & $x=0.4$ & $*$ & $*$ \\
\hline \multirow{5}{*}{$20-1$} & Baseline & 37.2662 & 2.4129 \\
\hline & $x=0.1$ & 37.2496 & 2.4139 \\
\hline & $x=0.2$ & 37.2560 & 2.4135 \\
\hline & $x=0.3$ & 37.2355 & 2.4148 \\
\hline & $x=0.4$ & 37.2104 & 2.4164 \\
\hline \multirow{5}{*}{$\begin{array}{c}020 \text { S1 } \\
\text { or } \\
11-2 \text { S2 }\end{array}$} & Baseline & 39.5237 & 2.2801 \\
\hline & $x=0.1$ & 39.4792 & 2.2826 \\
\hline & $x=0.2$ & 39.3936 & 2.2874 \\
\hline & $x=0.3$ & 39.3702 & 2.2887 \\
\hline & $x=0.4$ & 39.3288 & 2.2910 \\
\hline \multirow{5}{*}{202} & Baseline & 43.6166 & 2.0752 \\
\hline & $x=0.1$ & $*$ & $*$ \\
\hline & $x=0.2$ & 43.4140 & 2.0844 \\
\hline & $x=0.3$ & 43.4541 & 2.0826 \\
\hline & $x=0.4$ & 43.3717 & 2.0863 \\
\hline \multirow{5}{*}{203} & Baseline & $*$ & $*$ \\
\hline & $x=0.1$ & $*$ & $*$ \\
\hline & $x=0.2$ & 48.4509 & 1.8788 \\
\hline & $x=0.3$ & 48.4096 & 1.8803 \\
\hline & $x=0.4$ & 48.4645 & 1.8783 \\
\hline \multirow{5}{*}{220} & Baseline & 55.5662 & 1.6526 \\
\hline & $x=0.1$ & 55.4622 & 1.6554 \\
\hline & $x=0.2$ & 55.3569 & 1.6583 \\
\hline & $x=0.3$ & 55.2381 & 1.6616 \\
\hline & $x=0.4$ & 55.3091 & 1.6596 \\
\hline
\end{tabular}

Figure 20 summarizes the XRD spectrum for all $\mathrm{Bi}$ doping levels explored; these XRD measurements were performed to the pellet samples once sintered. In this figure it is evident that there is texturing of the materials, the dominant $00 \mathrm{O}$ reflections indicate that the alignment of the grains is preferred and 
lamellae stack perpendicular to the $c$ axis. This is also confirmed in the microstructure features as seen from the samples cross sections.

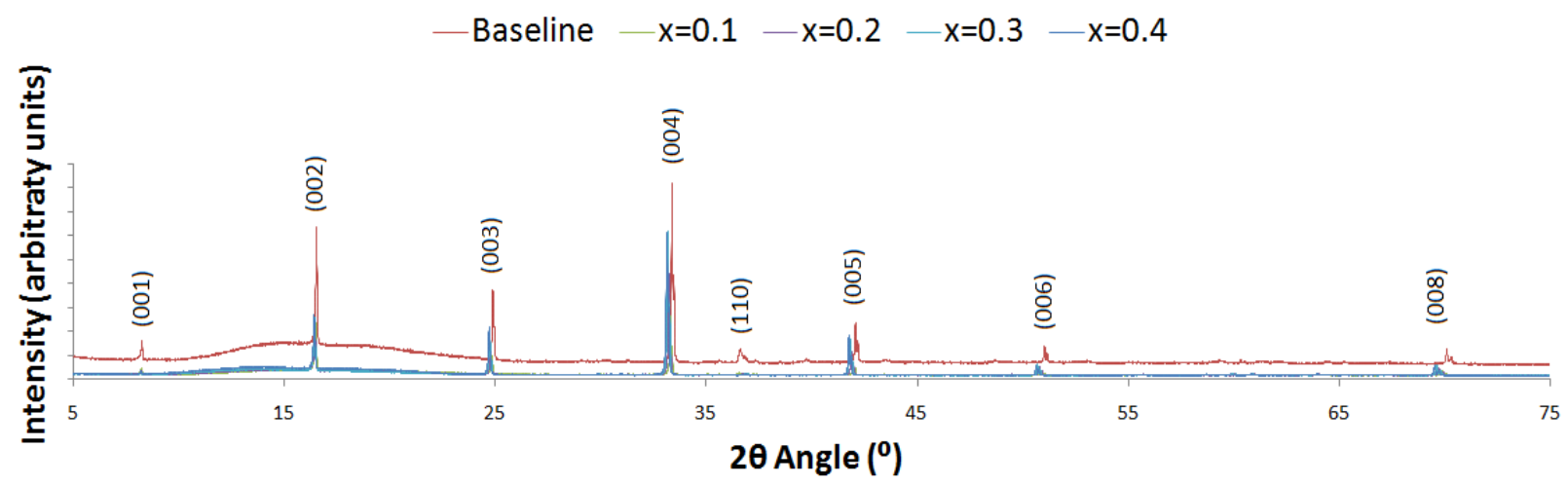

Figure 20. XRD spectrum for baseline and Bi doped CCO samples $\mathrm{x}=0.1,0.2,0.3,0.4$.

\subsubsection{SEM imaging}

Crystal textured samples have been obtained for all the compositions. The top view of the pressed planes shows disc shaped grains. During the sample pressing, the force of $1 \mathrm{GPa}$ is exerted directly over such planes. The porosity of the sample seems to decrease with increase of Bi doping. The density values also evidence this feature.

For the Bi concentration of 0.4, very large (larger than $100 \mu \mathrm{m}$ ) plate like grains (look like needles in the cut) grow across the pellet sample as seen from the plan view and the cross section images. For the top view, there are also smaller disc shaped grains. For all other compositions with Bi content 0 to 0.3 no needle shaped large grains are observed.

The grain size also changes with different doping concentrations. Whereas for no Bi doping (baseline) the grain size is $\sim 1.5 \mu \mathrm{m}$, the grains grow larger with increasing Bi concentration. The average grain size for $\mathrm{Bi} 0.1$ is $\sim 3.5 \mu \mathrm{m}$, for $\mathrm{Bi} 0.2$ is $\sim 6 \mu \mathrm{m}$, for $\mathrm{Bi} 0.3$ is $\sim 8 \mu \mathrm{m}$ and for $\mathrm{Bi} 0.4$ is found to be $\sim 9 \mu \mathrm{m}$ for the disc shaped grains and greater than $100 \mu \mathrm{m}$ for the needle shaped grains as mentioned above. 
CHAPTER 3. EFFECT OF Bi DOPING ON THE Ca-SITE $\left(\mathrm{Ca}_{3-x} \mathrm{Bi}_{x} \mathrm{Co}_{4} \mathrm{O}_{9}\right)$ ON THE THERMOELECTRIC PERFORMANCE AND NANOSTRUCTURE
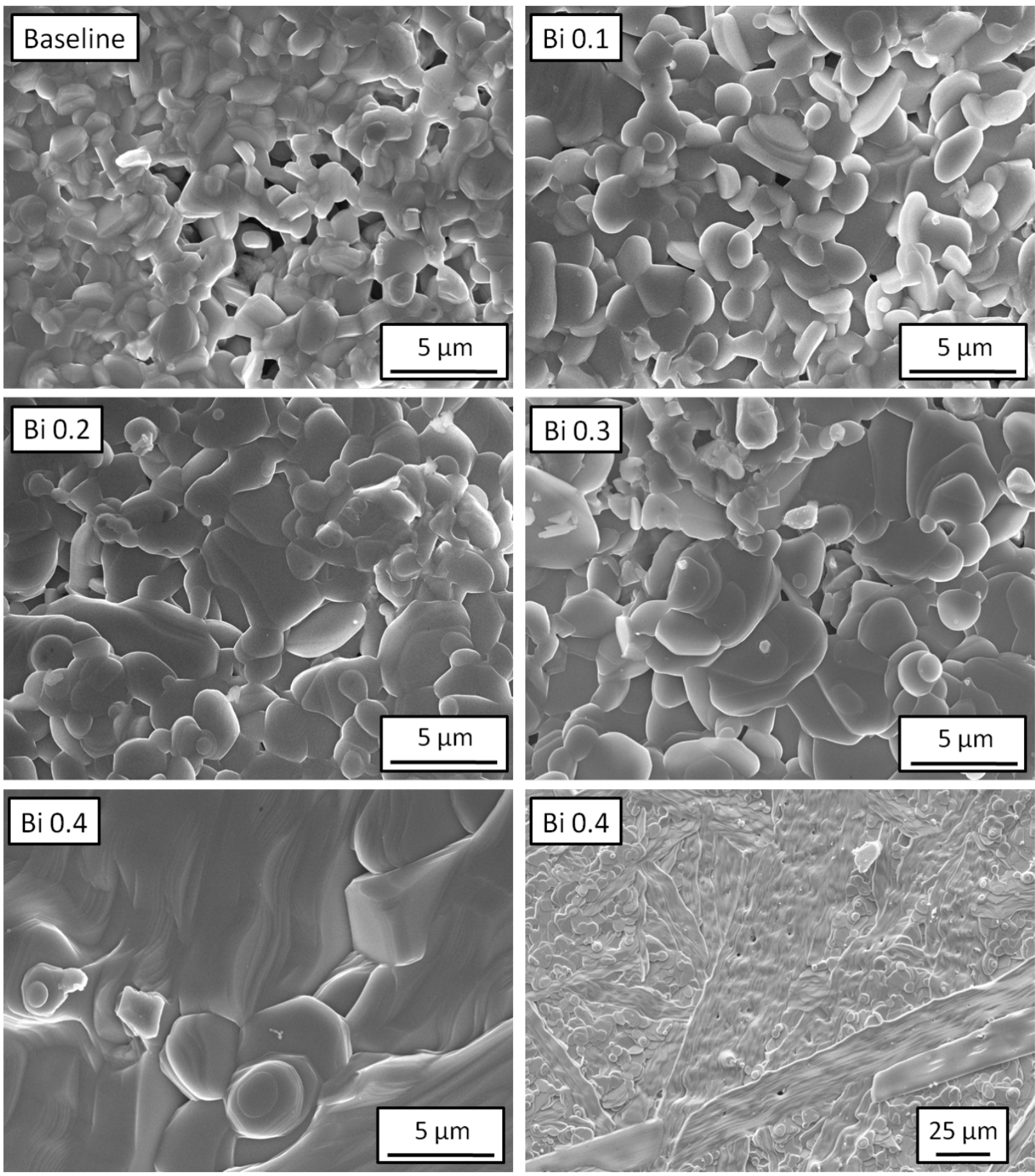

Figure 21. Pressed plane of baseline and Bi doped CCO pellets. Pressed at $1 \mathrm{GPa}$ and $150^{\circ} \mathrm{C}$ for 40 minutes. 
CHAPTER 3. EFFECT OF Bi DOPING ON THE Ca-SITE $\left(\mathrm{Ca}_{3-x} \mathrm{Bi}_{x} \mathrm{Co}_{4} \mathrm{O}_{9}\right)$ ON THE THERMOELECTRIC PERFORMANCE AND NANOSTRUCTURE
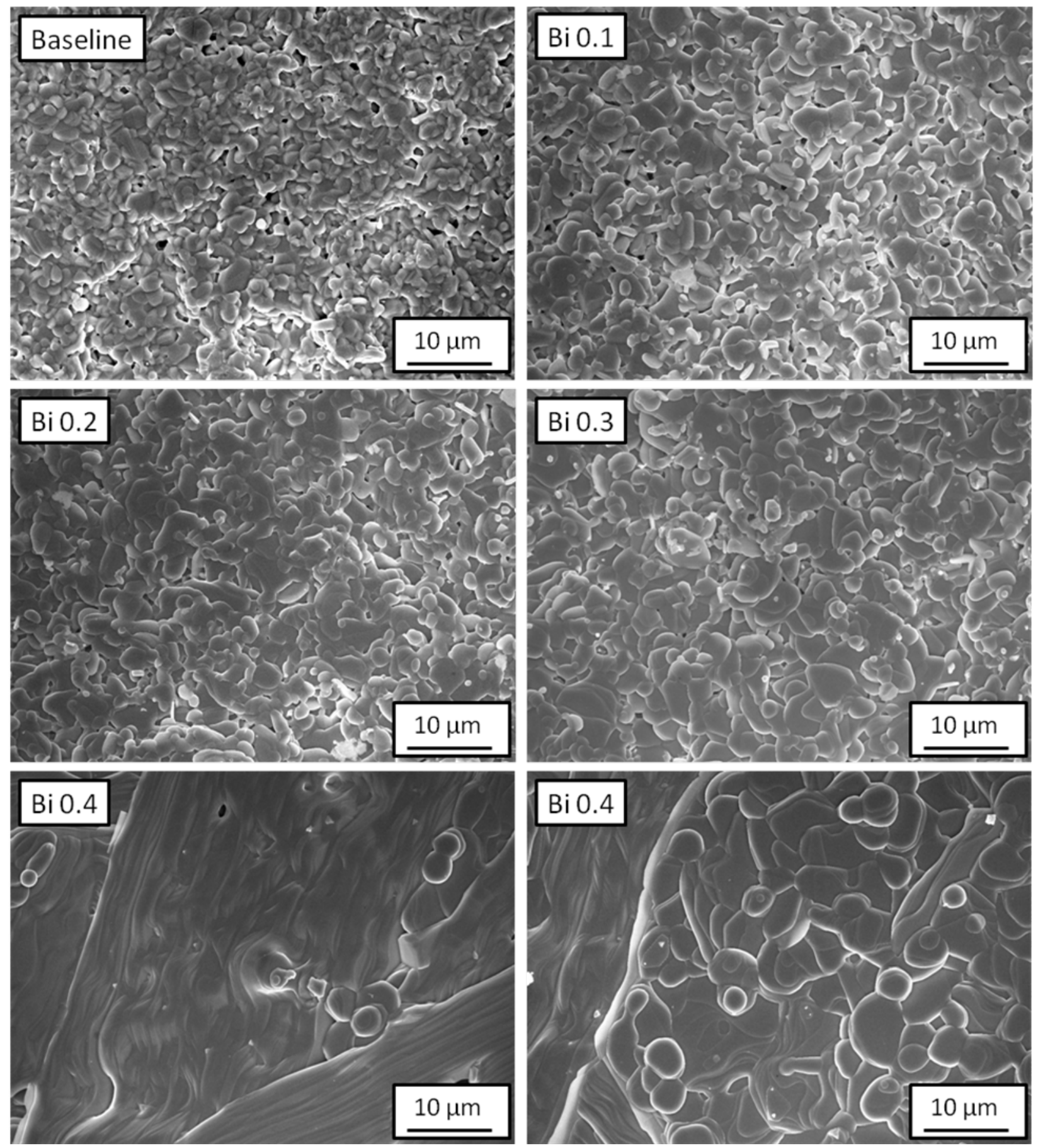

Figure 22. Pressed plane of baseline and Bi dopped CCO pressed at $1 \mathrm{GPa}$ and $150^{\circ} \mathrm{C}$ (lower magnification). 
The cross section shows very clean crystal textured samples. The horizontal direction coincides with the in-plane direction, i.e. it is perpendicular to the pressing axis when formation of the pellets takes place. The alignment is uniform, except for Bi 0.4 where the needle appearance-platelet shaped grains grow diagonally across large portions of the sample. The grains are visibly aligned along the in-plane direction and strong evidence of porosity for all the samples is observed.

For the Bi 0.4 doping level, the grain growth is triggered by $\mathrm{Bi}$ addition in such a way that alignment is negatively affected.

Despite of some porosity, the Bi 0.4 samples shows high packing of the grains in the needle appearance structures as shown in Figure 23. Disc shaped grains grow larger and fuse with neighboring grains to form them. Alignment along the in-plane direction is observable in the horizontal smaller grains for this composition. 
CHAPTER 3. EFFECT OF Bi DOPING ON THE Ca-SITE $\left(\mathrm{Ca}_{3-x} \mathrm{Bi}_{x} \mathrm{Co}_{4} \mathrm{O}_{9}\right)$ ON THE THERMOELECTRIC PERFORMANCE AND NANOSTRUCTURE
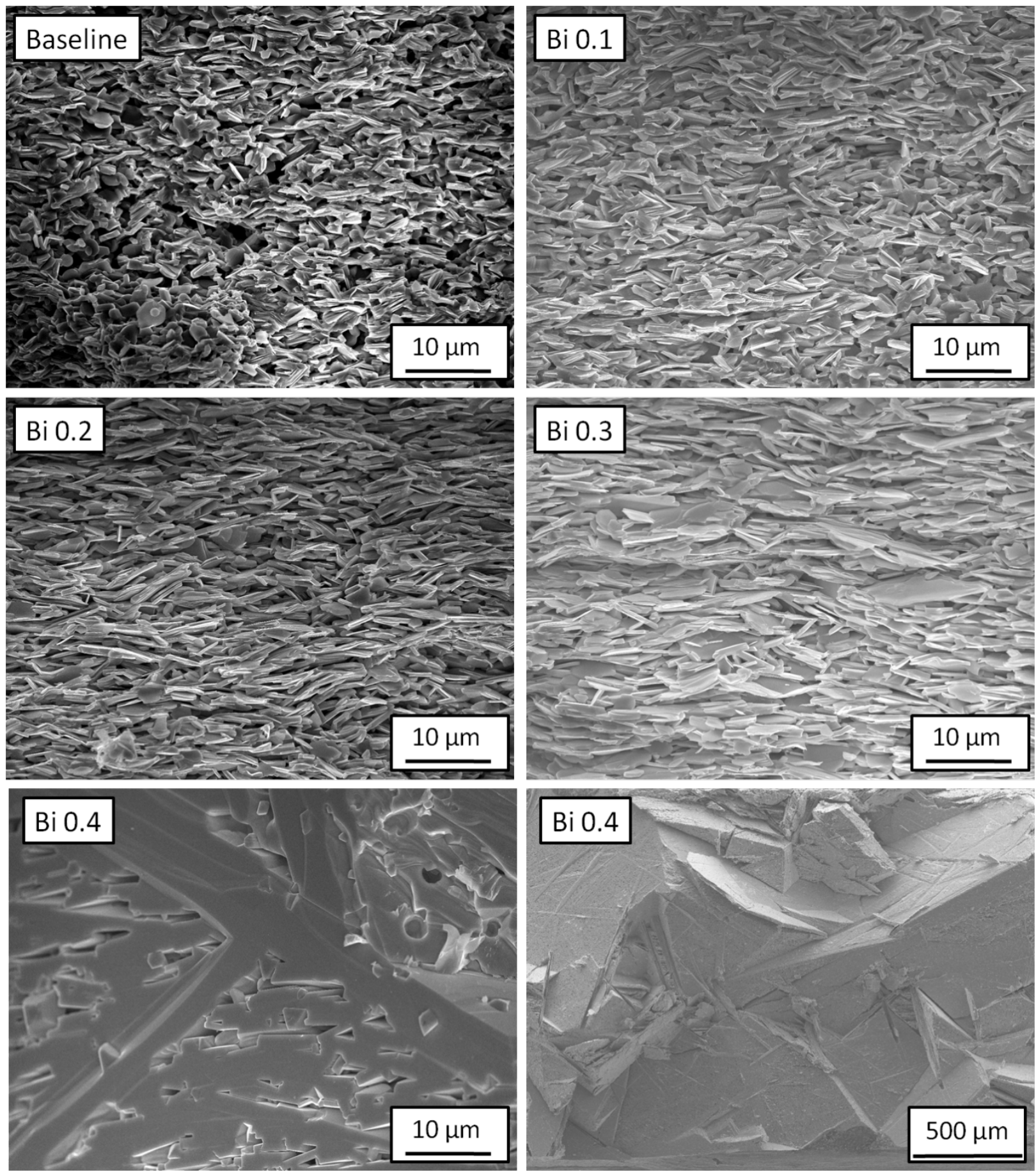

Figure 23. Cross section of baseline and Bi doped CCO pellets. Pressed at $1 \mathrm{GPa}$ and $150^{\circ} \mathrm{C}$ for 40 minutes. In plane direction coincides with horizontal direction, perpendicular to pressing axis. 
Compositions of $\mathrm{Bi} 0$ to 0.3 show similar microstructure. Alignment is somehow improved by $\mathrm{Bi}$ addition; the baseline cross section shows very disordered grains and a slight tendency to alignment, as the $\mathrm{Bi}$ doping is increased a stronger tendency to alignment is observed.

The samples with Bi doping levels of 0.3 and 0.4 are shown in Figure 24 and Figure 25 respectively. For the TEM images three main types of contrast can be observed: mass contrast, thickness contrast and diffraction contrast. The mass contrast has to do with the atomic weight of the elements, thus lighter and heavier atoms can be distinguished. The thickness contrast depends on how thin or thick are the samples, the electron beam passing through the sample will have more or less intensity depending on that. Finally, the diffraction contrast comes from the difference in the orientation of the crystals in the sample; the electrons encountering the crystals will diffract accordingly with the crystal plane orientations and the resulting image will account for such differences. In both figures, the lamellar structure of the CCO grains can be observed. The contrast between lamellae is of diffraction nature; furthermore, the homogeneity of the composition has been confirmed from EDS analysis. Notice that the $c$ axis direction is perpendicular to the lamellae. In the diffraction pattern, the intensity spots are characteristic of the CCO system.

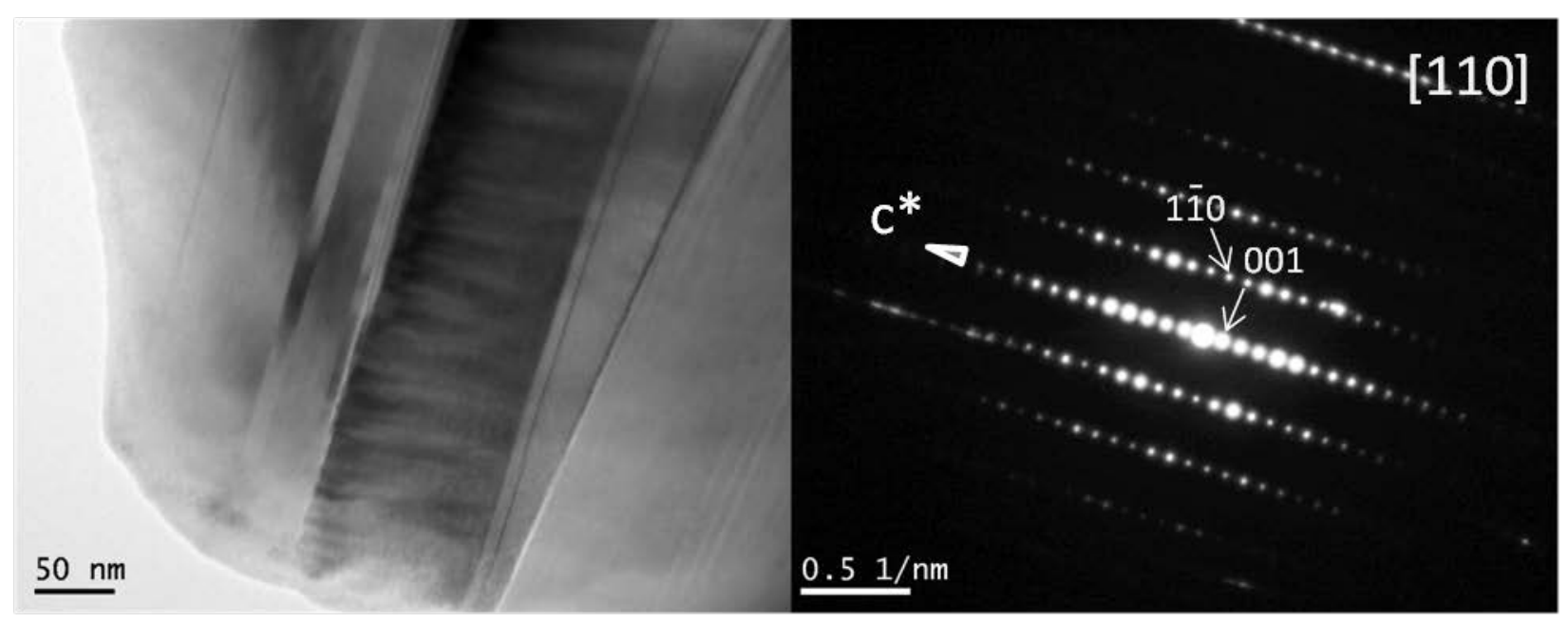

Figure 24. TEM image and diffraction pattern along the [110] zone axis for the Bi 0.3 doped samples. 


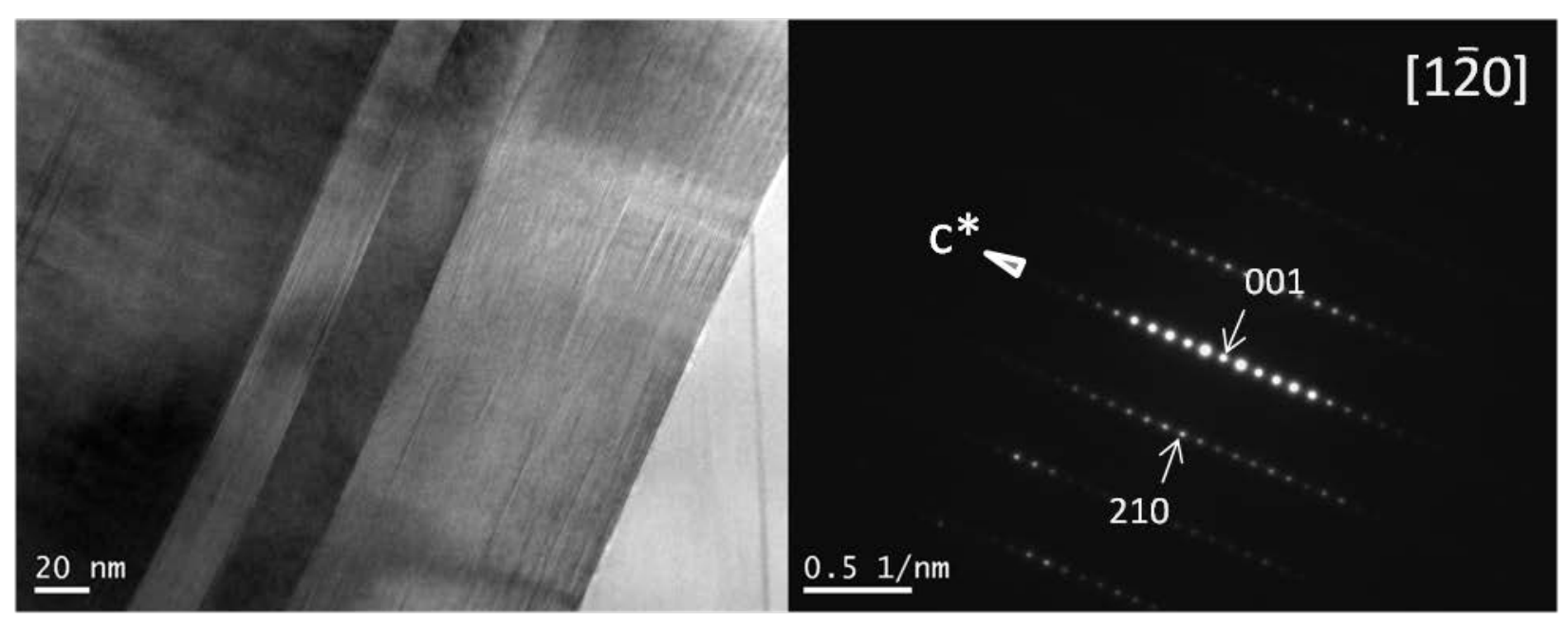

Figure 25. TEM image and diffraction pattern along the $\left[\overline{1}^{2} 0\right]$ zone axis for the Bi 0.4 doped samples.

These microstructure features (alignment, higher packing, grain growth) associated to Bi addition can lead to reduction of electrical resistivity as suggested by Xu et. al. 2002 [27].

\subsubsection{Transport Properties}

The transport properties for the different Bi doped samples are shown in Figure 26. As expected they are temperature dependent; however, the electrical resistivity tends to remain constant through the entire temperature range except for the samples with $\mathrm{Bi} x=0.4$ composition.

The Seebeck coefficient maximum is obtained around $1050 \mathrm{~K}$ for $\mathrm{Bi}=0.3$ doped samples. It can be seen that the addition of $\mathrm{Bi}$ to the $\mathrm{CCO}$ system reduces the Seebeck coefficient for samples with Bi doping 0.1 to 0.3 from room temperature to $950 \mathrm{~K}$ and then slightly increases it. For samples with Bi content of 0.4 , the Seebeck coefficient is reduced over the entire range of temperature.

The electrical resistivity is evidently changed by $\mathrm{Bi}$ addition. For concentrations below $0.3, \mathrm{Bi}$ substitution of Ca sites reduces the electrical resistivity; this result is in good agreement with those reported by $\mathrm{Xu}$ et. al. 2002.[27] Particularly for Bi 0.3 concentration the resistivity reaches its minimum and above that it increases drastically. 
The power factor shows the effect contribution of both Seebeck coefficient and electrical resistivity together. As seen from Figure 26, the addition of $\mathrm{Bi}$ into the CCO system is advantageous for $\mathrm{Bi}$ concentrations of 0.2 and 0.3 .

The thermal conductivity is also affected by $\mathrm{Bi}$ addition. The lowest thermal conductivity is found for $\mathrm{Bi}$ concentrations of 0.2 and 0.3 over the entire temperature range. For concentrations of 0.1 , thermal conductivity is found to be reduced from the reference value but not very significantly. In the case of $\mathrm{Bi}$ 0.4 the thermal conductivity increased, this behavior was somehow expected in relation to the high density of the compound triggered by Bi addition.
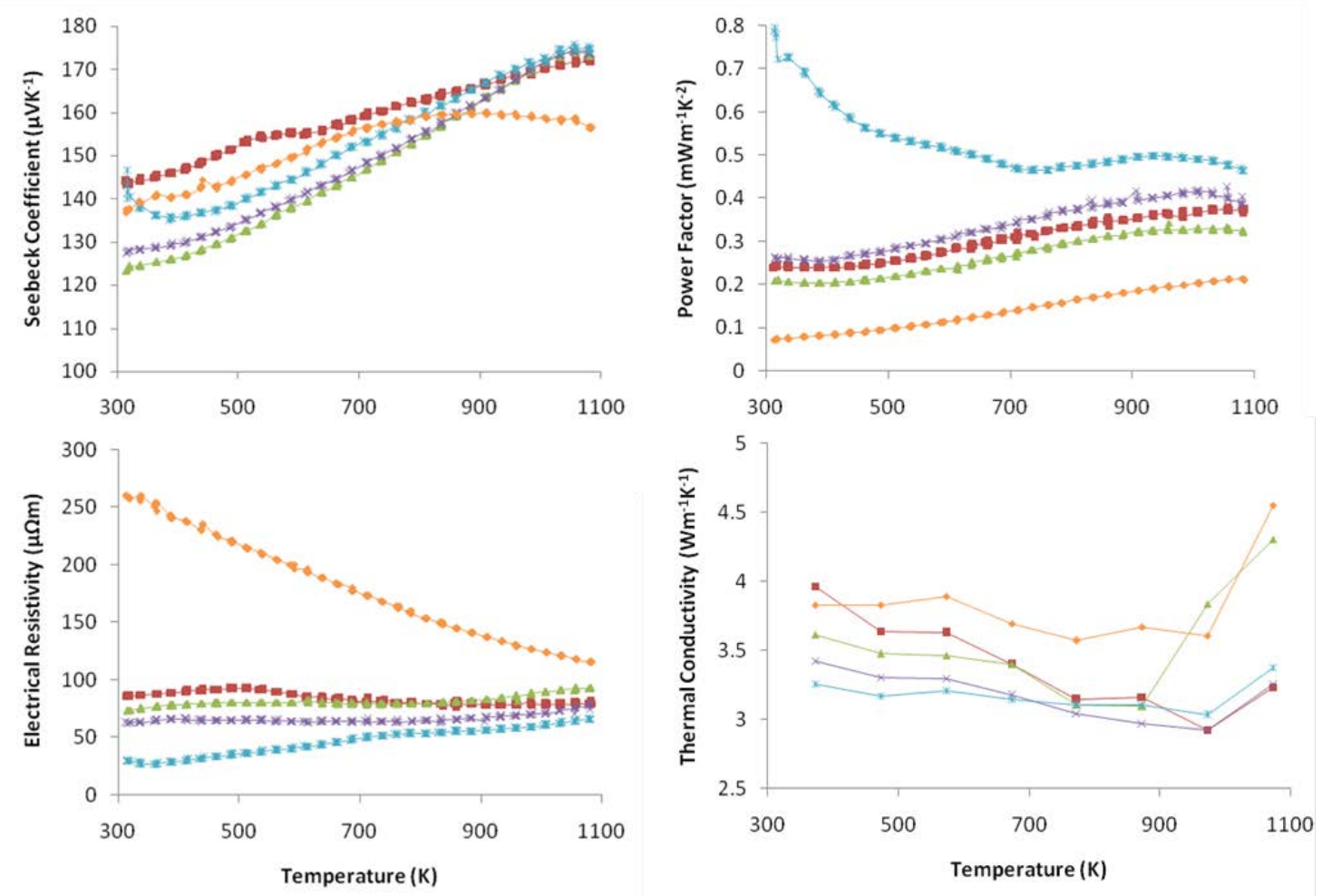

$\rightarrow$ Baseline $\rightarrow \mathrm{x}=0.1 \rightarrow \mathrm{x}=0.2 \rightarrow \mathrm{x}=0.3 \rightarrow \mathrm{x}=0.4$

Figure 26. Transport properties for the baseline and Bi doped CCO samples $x=0.1,0.2,0.3$ and 0.4 Pressed at $1 \mathrm{GPa}$ and $150^{\circ} \mathrm{C}$.

The dimensionless figure of merit ZT for the Bi doped CCO samples is shown in Figure 27, it can be clearly seen that the best performance is given by Bi doping concentration of 0.3 for which ZT maximum 
value is 0.16 at $973 \mathrm{~K}$. Bi addition to CCO in 0.3 concentration lead to $29 \%$ improvement of ZT above the baseline better performance.

Li et. al. 2000 report ZT values of 0.14 at $973 \mathrm{~K}$ [28], in their research this value is obtained for the 0.5 doping level. However from Figure 27 it is seen that for Bi doping levels higher than 0.3 , detriment of the thermoelectric performance is observed. Further refinement on the range between 0.2 and $0.4 \mathrm{Bi}$ doping should be examined to obtain the specific doping concentration for which the ZT is maximum.
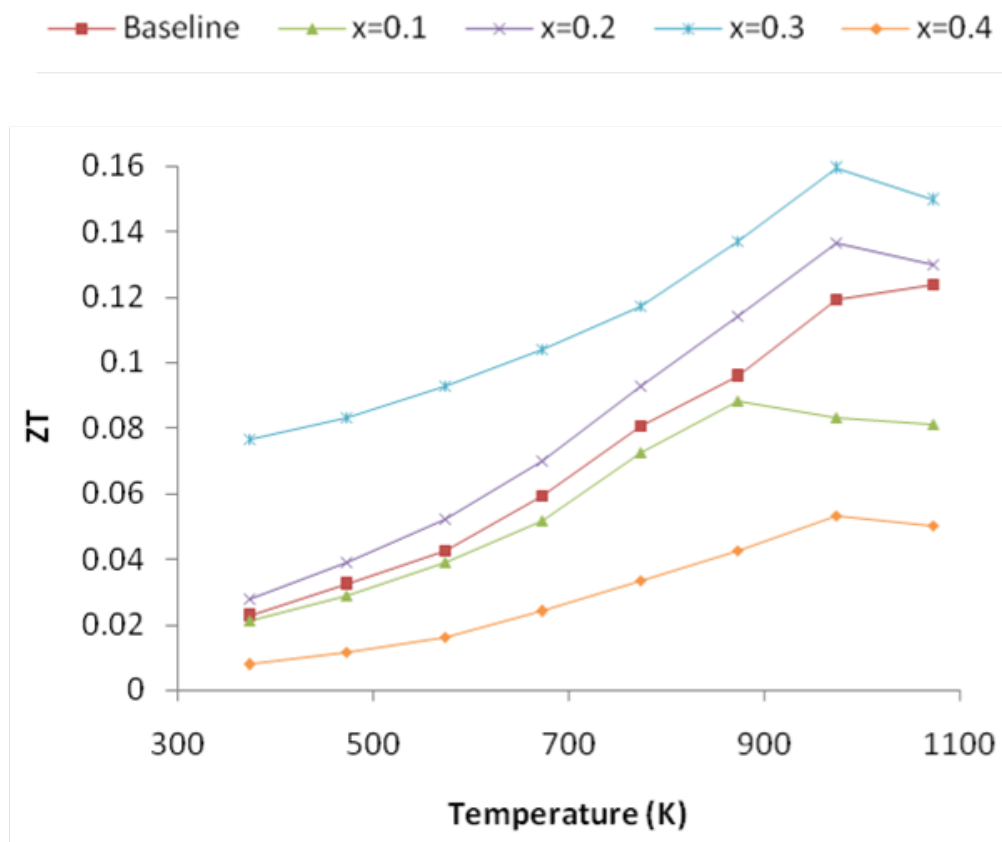

Figure 27. Dimensionless figure of merit for the baseline and Bi doped CCO samples $x=0.1,0.2,0.3,0.4$.

\subsubsection{Nanostructure and Impurity Segregation}

There are features (see regions circled in Figure 28) that show evidence of cation segregation at various grain boundaries. Two types of grain boundaries are identified. Type I grain boundary is the grain boundary in between the nano-lamellae that share the same $c$-axis, this type of grain boundary contains dislocation features that repeat in pairs periodically. Each pair of dislocations constitutes one repeating unit (See Figure 30). Type II grain boundary is the grain boundary in between the nano-lamellae that have different $c$-axis.

Detailed EDS analysis is summarized in Table 4. In the grain boundary Type I; dislocations are observed near the grain-lamellae boundary. The bright spots in the grain boundary represent Bi atoms that 
accumulate in the wider regions of the dislocation zones. The EDS analysis points of the area are shown in Figure 29; for the spot marked as 1 in Type I, the Bi content is approximately 1.65 atomic percent; and for the bright region on the grain boundary (spot 2) the Bi concentration is approximately 3.33 atomic percent.

In the grain boundary Type II, diamond shaped features are observed. These features are of regular size and shape as seen in Figure 28 and Figure 29. The brighter diamond shape features present higher $\mathrm{Bi}$ concentrations. The EDS analysis shows that segregation of $\mathrm{Bi}$ in the bright diamond shaped regions occur; in the spot marked as 1 in Type II, the Bi content is approximately 2.15 atomic percent; for spot 2 the $\mathrm{Bi}$ concentration is 5.24 atomic percent and for sport 3 the Bi concentration is 1.53 atomic percent.

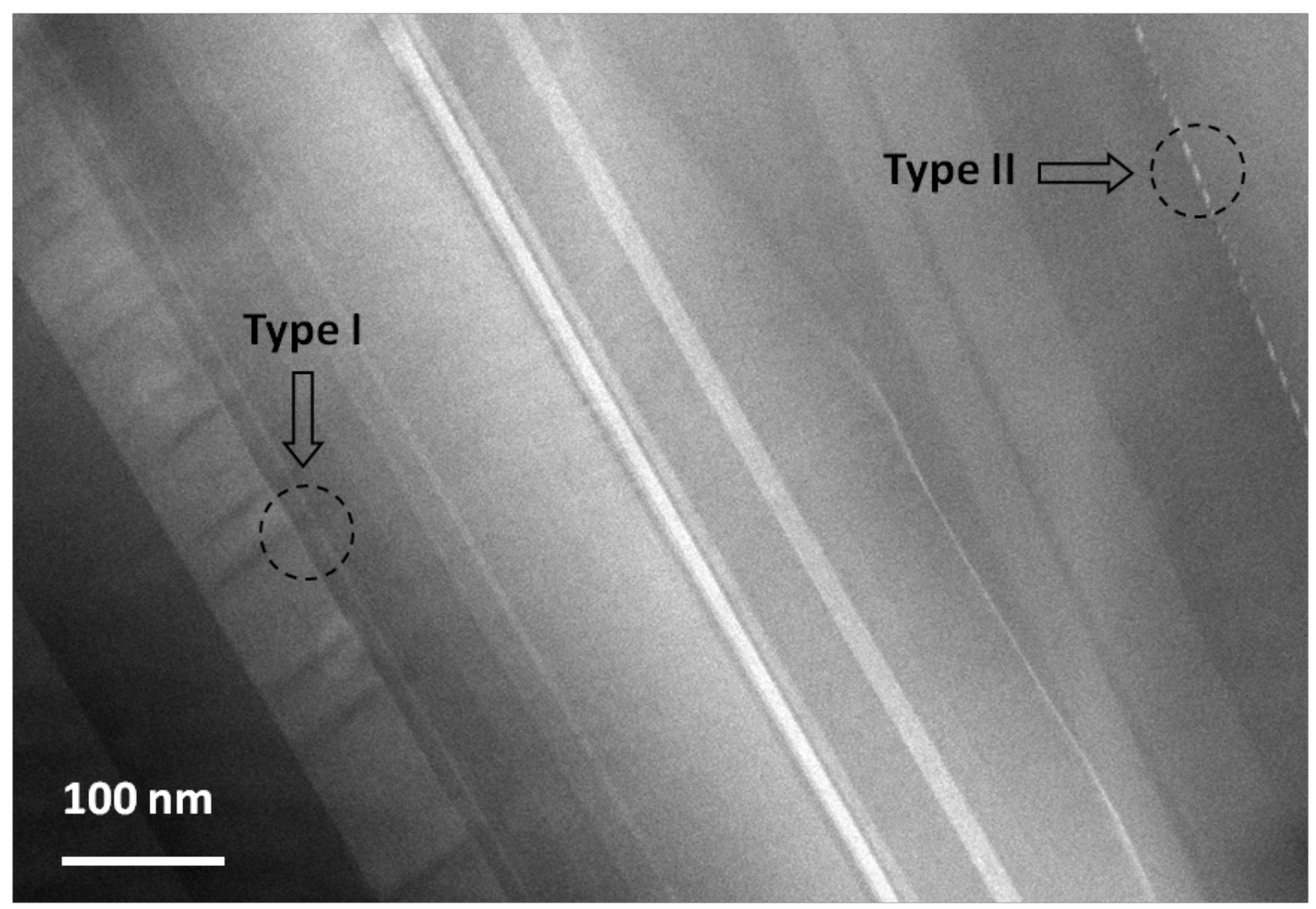

Figure 28. STEM image of CCO - Bi 0.3 doped sample showing Bi segregation features in grain boundaries type I and II. 
CHAPTER 3. EFFECT OF Bi DOPING ON THE Ca-SITE $\left(\mathrm{Ca}_{3-x} \mathrm{Bi}_{x} \mathrm{CO}_{4} \mathrm{O}_{9}\right)$ ON THE THERMOELECTRIC PERFORMANCE AND NANOSTRUCTURE
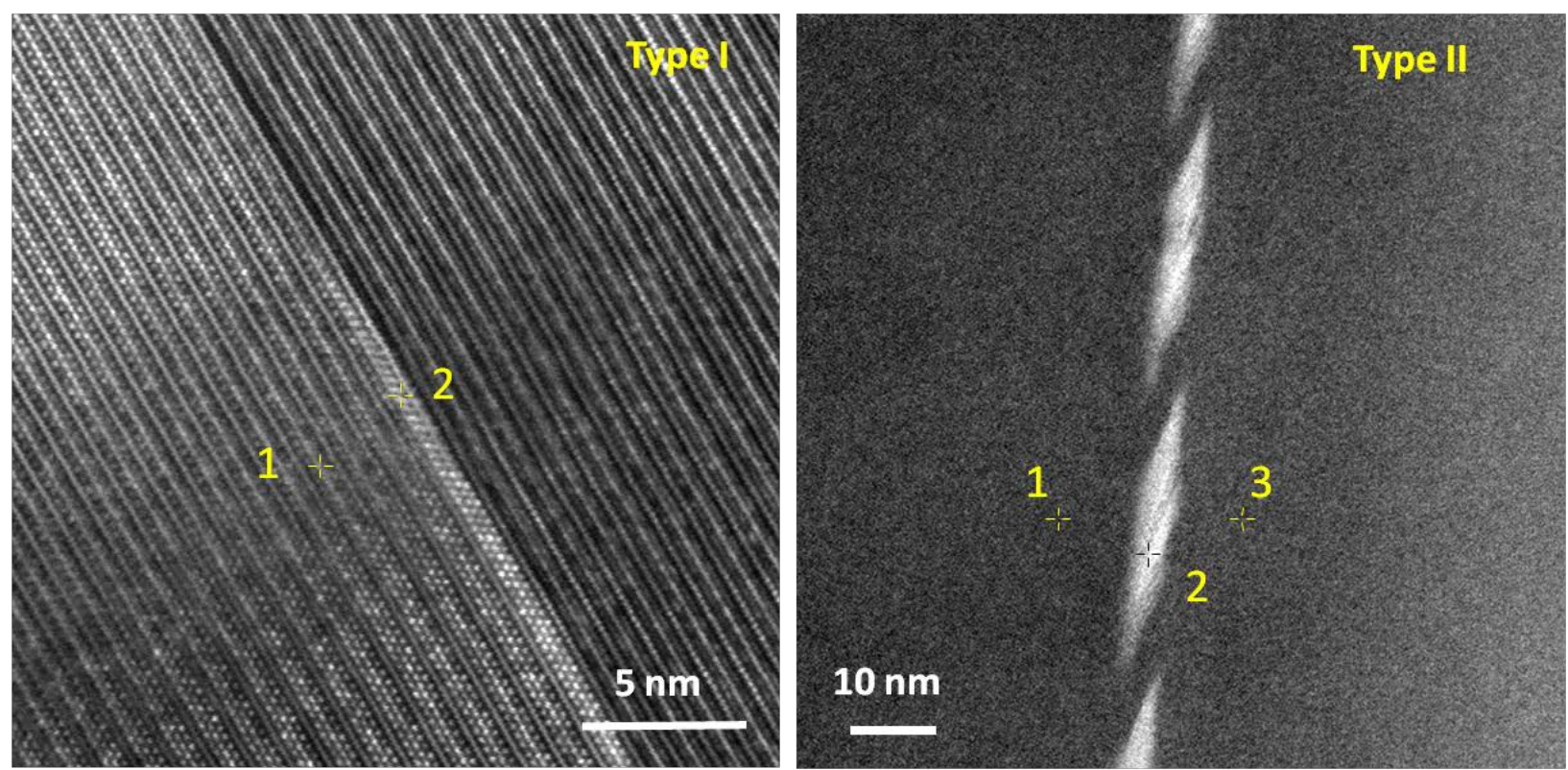

Figure 29. STEM images of CCO - Bi 0.3 doped samples pressed at $1 \mathrm{GPa}$. Bi segregation occurs at the boundaries between lamellae. Type II grain boundary is off axis.

Table 4. EDS analysis of the Bi segregation regions as depicted in Figure 29.

\begin{tabular}{cccccc}
\hline \multicolumn{7}{c}{ Atomic \% } \\
\hline Grain Boundary & EDS & Ca & Co & O & Bi \\
\hline Type I & $\mathbf{1}$ & 22.00 & 19.73 & 56.63 & 1.65 \\
& $\mathbf{2}$ & 15.47 & 22.05 & 59.14 & 3.33 \\
\hline Type II & $\mathbf{1}$ & 23.00 & 22.31 & 52.54 & 2.15 \\
& $\mathbf{2}$ & 19.44 & 23.59 & 51.74 & 5.24 \\
& $\mathbf{3}$ & 22.21 & 24.26 & 51.99 & 1.53 \\
\hline
\end{tabular}




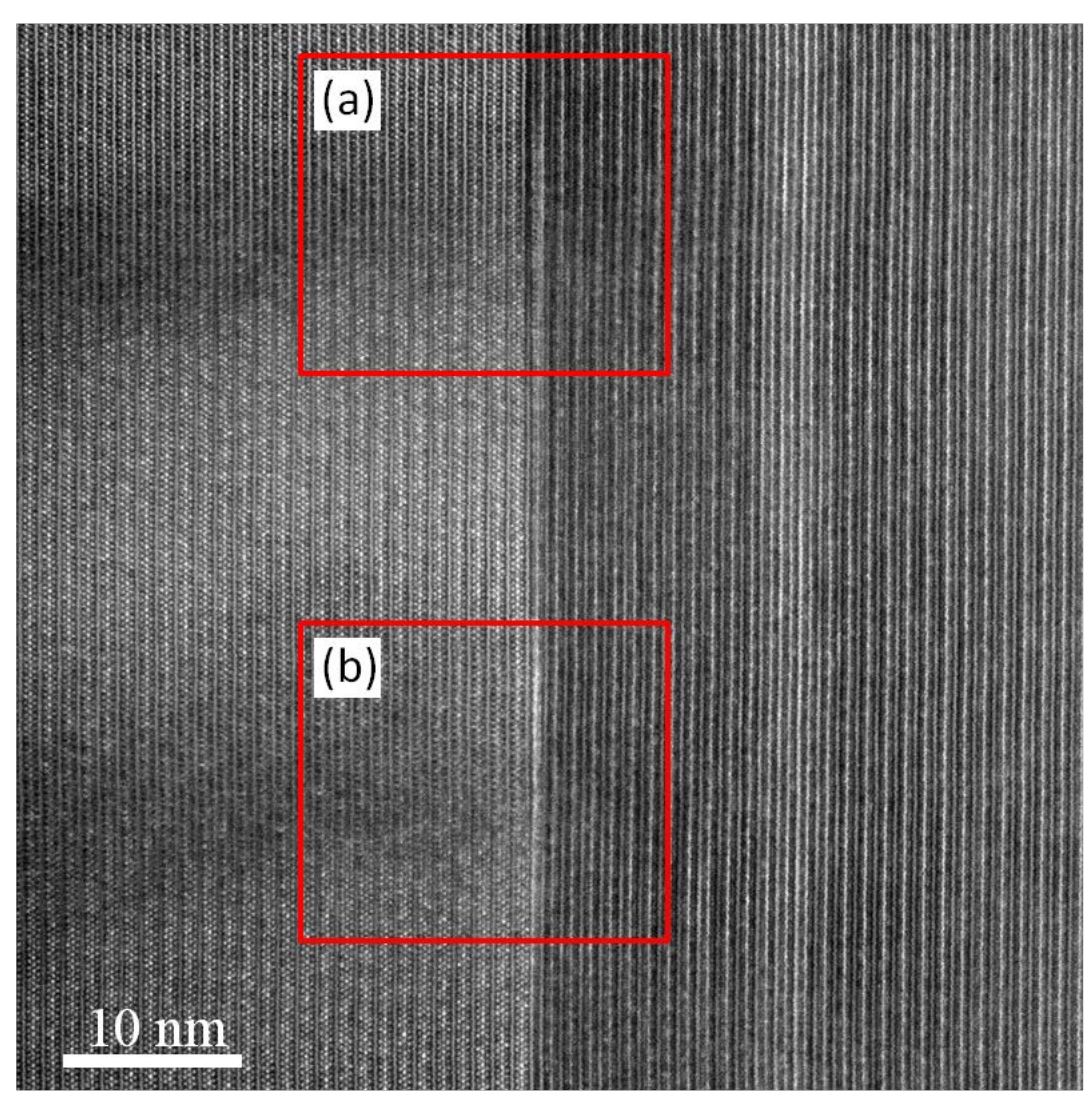

Figure 30. Type I grain boundary dislocation repeating unit, (a) Bi depleted region (dark contrast line), (b) Bi enriched region (bright contrast line).

In Figure 30, the repeating unit pair of dislocations from the type I grain boundary is shown, the high resolution images of regions (a) and (b) with the specific dislocations exhibiting different $\mathrm{Bi}$ content are shown in Figure 31 and Table 5. For region (a) EDS spots 4 and 5 show almost no Bi content whereas for region (b) spots 1 to 5 show enrichment of Bi content. 
CHAPTER 3. EFFECT OF Bi DOPING ON THE Ca-SITE $\left(\mathrm{Ca}_{3-x} \mathrm{Bi}_{x} \mathrm{Co}_{4} \mathrm{O}_{9}\right)$ ON THE THERMOELECTRIC PERFORMANCE AND NANOSTRUCTURE
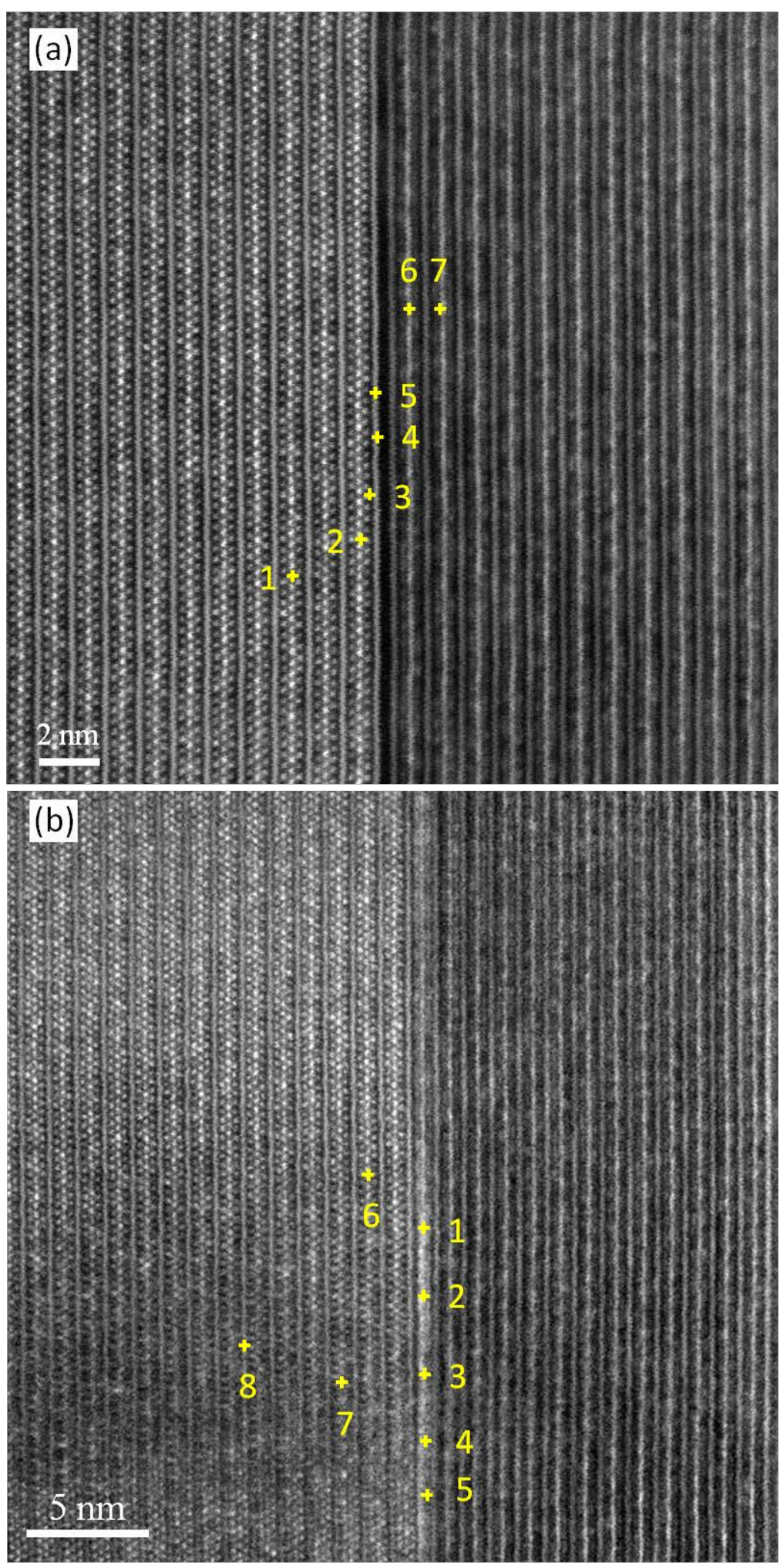

Figure 31. High resolution STEM images of the Bi depleted (a) and enriched (b) regions of the dislocations found in type I grain boundary. 
CHAPTER 3. EFFECT OF Bi DOPING ON THE Ca-SITE $\left(\mathrm{Ca}_{3-x} \mathrm{Bi}_{x} \mathrm{Co}_{4} \mathrm{O}_{9}\right)$ ON THE THERMOELECTRIC PERFORMANCE AND NANOSTRUCTURE

Table 5. EDS analysis of the dislocation regions (a) and (b) in Figure 31, grain boundary type I.

Atomic \%

\begin{tabular}{cccccc}
\hline Region & EDS & Ca & Co & O & Bi \\
\hline (a) & $\mathbf{1}$ & $21.28 \pm 0.74$ & $22.81 \pm 0.85$ & $54.73 \pm 1.44$ & $1.18 \pm 0.36$ \\
& $\mathbf{2}$ & $19.82 \pm 0.67$ & $25.46 \pm 0.86$ & $53.64 \pm 1.38$ & $1.07 \pm 0.33$ \\
& $\mathbf{3}$ & $20.97 \pm 0.71$ & $21.24 \pm 0.82$ & $56.38 \pm 1.42$ & $1.41 \pm 0.37$ \\
& $\mathbf{4}$ & $17.46 \pm 0.64$ & $24.49 \pm 0.90$ & $58.05 \pm 1.42$ & $0.00 \pm 0.00$ \\
& $\mathbf{5}$ & $19.55 \pm 0.67$ & $26.66 \pm 0.86$ & $53.79 \pm 1.38$ & $0.00 \pm 0.00$ \\
& $\mathbf{6}$ & $18.31 \pm 0.67$ & $24.19 \pm 0.89$ & $56.86 \pm 1.47$ & $0.65 \pm 0.29$ \\
& $\mathbf{7}$ & $18.08 \pm 0.68$ & $23.52 \pm 0.86$ & $57.37 \pm 1.48$ & $1.03 \pm 0.38$ \\
\hline $\mathbf{1})$ & $18.45 \pm 0.67$ & $18.69 \pm 0.73$ & $60.22 \pm 1.50$ & $2.63 \pm 0.50$ \\
& $\mathbf{2}$ & $15.47 \pm 0.66$ & $22.05 \pm 0.81$ & $59.14 \pm 1.48$ & $3.33 \pm 0.54$ \\
& $\mathbf{3}$ & $17.37 \pm 0.67$ & $22.15 \pm 0.85$ & $57.48 \pm 1.47$ & $3.01 \pm 0.53$ \\
& $\mathbf{4}$ & $17.55 \pm 0.69$ & $23.64 \pm 0.89$ & $56.48 \pm 1.50$ & $2.33 \pm 0.51$ \\
& $\mathbf{5}$ & $15.84 \pm 0.69$ & $25.83 \pm 0.94$ & $55.90 \pm 1.56$ & $2.44 \pm 0.53$ \\
& $\mathbf{6}$ & $19.36 \pm 0.68$ & $19.92 \pm 0.79$ & $59.69 \pm 1.47$ & $1.03 \pm 0.39$ \\
& $\mathbf{7}$ & $22.00 \pm 0.70$ & $19.73 \pm 0.80$ & $56.63 \pm 1.39$ & $1.65 \pm 0.37$ \\
& $\mathbf{8}$ & $17.81 \pm 0.63$ & $22.44 \pm 0.78$ & $58.41 \pm 1.40$ & $1.33 \pm 0.37$ \\
\hline
\end{tabular}

TEM images on axis of Type II grain boundary features are shown in Figure 32 . The diamond shape seen in Figure 29 results from an optical effect having the sample off axis, by tilting the sample better observation of these features was possible. In Figure 32 (a) the series of dislocations can be appreciated along the boundary between the lamellae. The diffraction pattern corresponds to the circled area; on its centre two diffraction patterns cross, notice that there is a small angle between them, this angle is the same formed by the two crystal planes converging at the dislocations.

The dislocations identified in grain boundaries Type I and II are of different nature. The mismatch between the different $a b$ planes of CCO lamellae sharing the same $c$ - axis direction gives rise to the dislocations seen in Type I grain boundaries; whereas dislocations in the grain boundary Type II arise from the mismatch between the angle at which the crystal planes of the two neighboring lamellae bundles join. In each case $\mathrm{Bi}$ atoms diffuse to the crystal defects generated by the lattice distortion as pointed out by Chen et. al. [61]. The energy associated to the grain boundary dislocations will be greater 
CHAPTER 3. EFFECT OF Bi DOPING ON THE Ca-SITE $\left(\mathrm{Ca}_{3-x} \mathrm{Bi}_{x} \mathrm{Co}_{4} \mathrm{O}_{9}\right)$ ON THE THERMOELECTRIC PERFORMANCE AND NANOSTRUCTURE

for the type of dislocations found in grain boundary Type II and also impurity segregation will be; EDS analysis of the Bi concentration in such areas also confirms this preferential segregation.
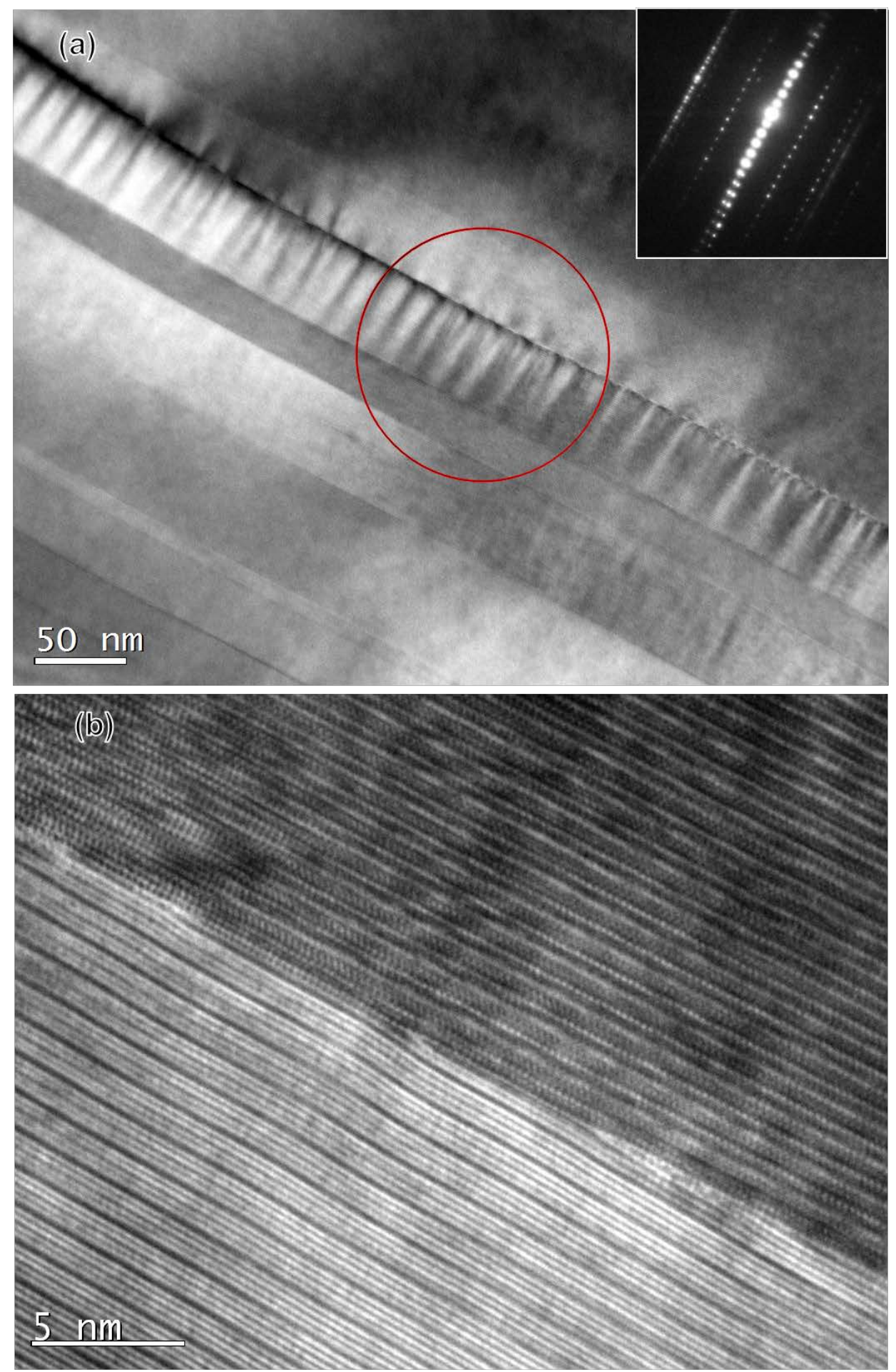

Figure 32. TEM images of the grain boundary Type II on axis. (a) Grain boundary with diffraction pattern of the circled area. (b) High resolution TEM image of the dislocations present between lamellae. 


\subsection{Effect of Pressing Conditions on Bi doped samples}

For Bi doping levels of 0.1 to 0.3 a second set of measurements were taken. The pressing conditions for the sample formation were changed to evaluate if further improvement of the Bi doped CCO system was possible. The samples were pressed at room temperature under $0.5 \mathrm{GPa}$, all the other parameters were kept identical.

\subsubsection{Density of the Pellets}

The corresponding density values obtained for this set of samples are shown in Table 6. It can be appreciated that there is a reduction on the density with the new conditions. The highest relative density obtained is $78 \%$ for $0.3 \mathrm{Bi}$ doping level. With this new pressing condition the relative densities decreased in $9 \%$ for the baseline, $16 \%$ for the $\mathrm{Bi} 0.1$, and $18 \%$ for the 0.2 and $0.3 \mathrm{Bi}$ doped systems.

Table 6. Density values for the CCO system, baseline and Bi doped series pressed at $0.5 \mathrm{GPa}$ and $25^{\circ} \mathrm{C}$.

\begin{tabular}{lccc}
\multicolumn{2}{c}{ Sample } & Density $\left(\mathrm{g} / \mathrm{cm}^{3}\right)$ & Relative Density (\%) \\
\hline Single crystal CCO & & 4.68 & 100 \\
Baseline $\mathbf{0 . 5} \mathrm{GPa}$ & & 3.60 & 77 \\
& $\mathbf{X}$ & & 72 \\
$\mathrm{Bi}$ & 0.1 & 3.38 & 72 \\
& 0.2 & 3.35 & 78 \\
\hline
\end{tabular}

\subsubsection{SEM imaging}

In the microstructure, the change of the pressing conditions is also evident. For the baseline and the $\mathrm{Bi}$ 0.1 levels the pressed plane micrographs do not show much difference; however increased porosity is appreciated from the pressed plane and confirmed from the cross sectional images. On the other hand, for $\mathrm{Bi}$ doping levels of 0.2 and 0.3 , there is also difference in the shape of the pressed grains as seen from Figure 33, the shape is mostly circular; whereas for the previous pressing conditions the shape was more irregular and the grains were rather distorted.

The grain size for the baseline is approximately $1.5 \mu \mathrm{m}$. For the Bi 0.1 doped samples is $\sim 2.5 \mu \mathrm{m}$, for the 0.2 is $\sim 3 \mu \mathrm{m}$ and for the $0.3 \mathrm{Bi}$ doped samples is $\sim 4.5 \mu \mathrm{m}$. The pressing conditions clearly show effect in the grain growth, for all the doping levels the grain size is smaller with the lower pressure processing. This pressing - grain growth relation is important and agrees with the density values obtained for the 
CHAPTER 3. EFFECT OF Bi DOPING ON THE Ca-SITE $\left(\mathrm{Ca}_{3-x} \mathrm{Bi}_{x} \mathrm{Co}_{4} \mathrm{O}_{9}\right)$ ON THE THERMOELECTRIC PERFORMANCE AND NANOSTRUCTURE

different set of samples studied; moreover it is concomitantly attached to the porosity of the samples. From the SEM images of the pressed planes (Figure 33 and Figure 34) and cross section (Figure 35), the increase in porosity results evident with the lower pressure forming conditions.
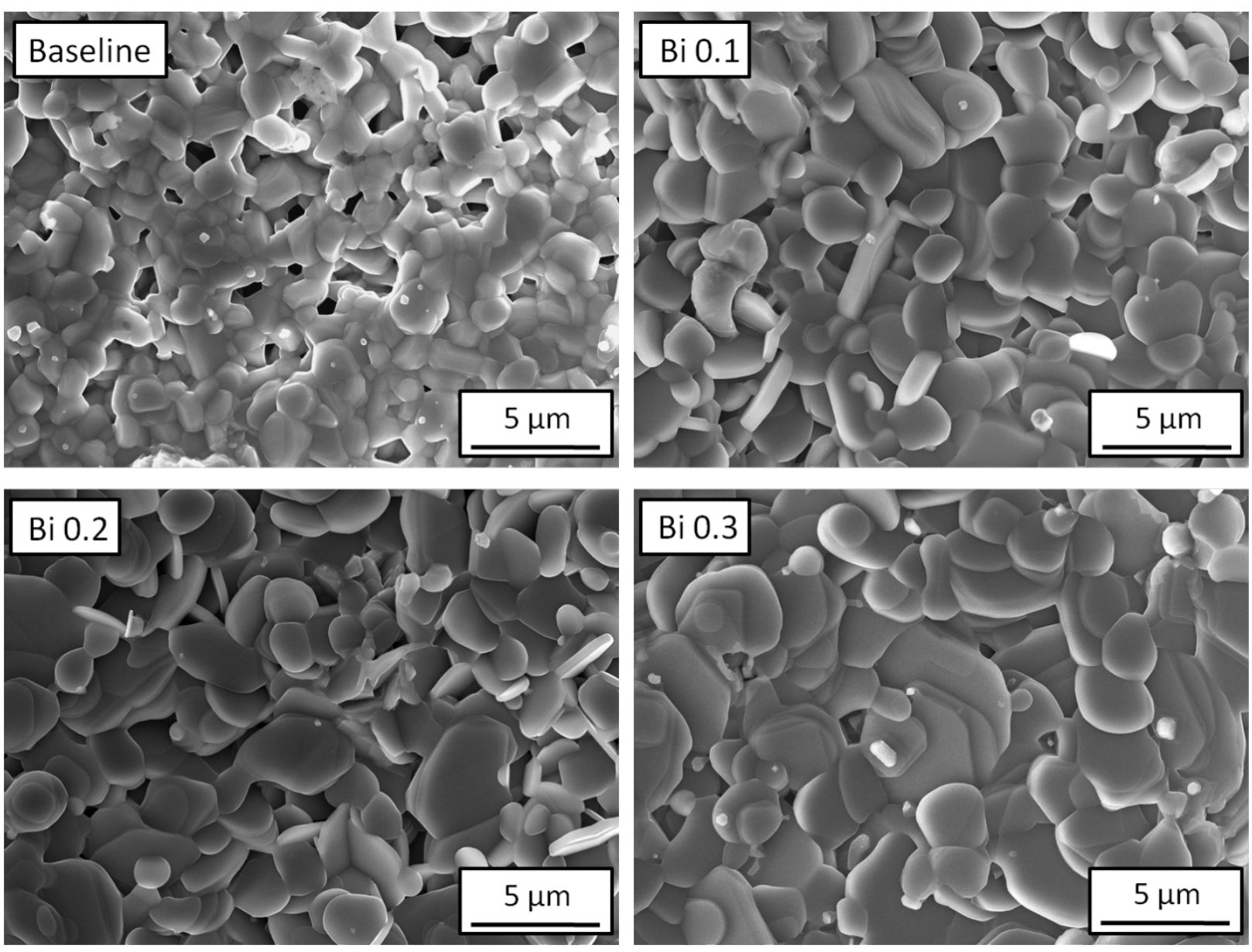

Figure 33. Pressed plane of baseline and Bi doped CCO pellets. Pressed at $0.5 \mathrm{GPaand} 25^{\circ} \mathrm{C}$ for 40 minutes. 

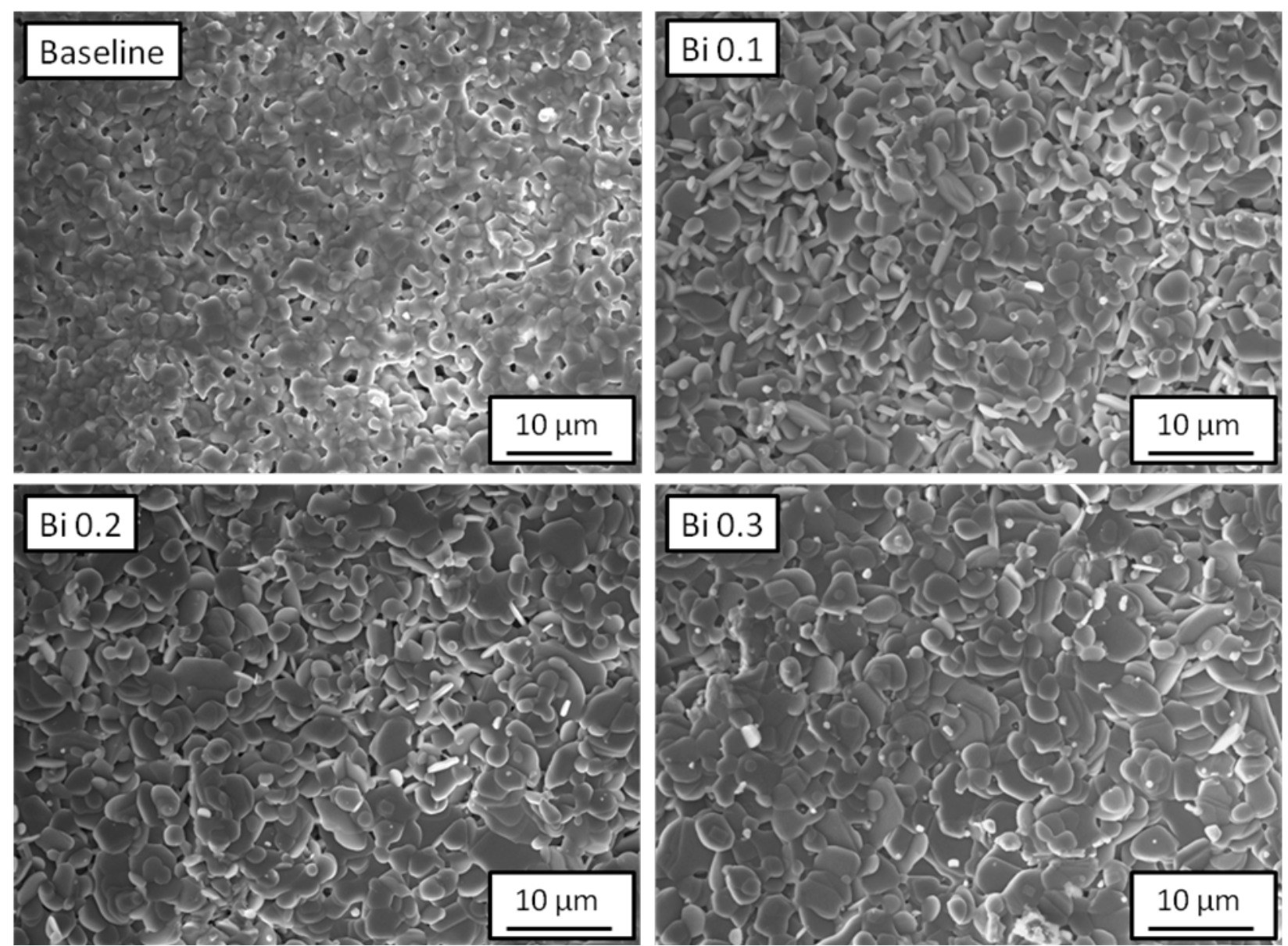

Figure 34. Pressed plane of baseline and Bi dopped CCO pressed at $0.5 \mathrm{GPa}$ and $25^{\circ} \mathrm{C}$ (lower magnification).

The alignment of the CCO grains in the sample can be seen in the cross section SEM images. The samples look uniform throughout all areas. It can be appreciated that the alignment is increased with the $\mathrm{Bi}$ doping level as noticed also for the $1 \mathrm{GPa}$ pressed set. Again, the aligned grains coincide with the horizontal plane, perpendicular to the pressing axis. 
CHAPTER 3. EFFECT OF Bi DOPING ON THE Ca-SITE $\left(\mathrm{Ca}_{3-x} \mathrm{Bi}_{x} \mathrm{Co}_{4} \mathrm{O}_{9}\right)$ ON THE THERMOELECTRIC PERFORMANCE AND NANOSTRUCTURE
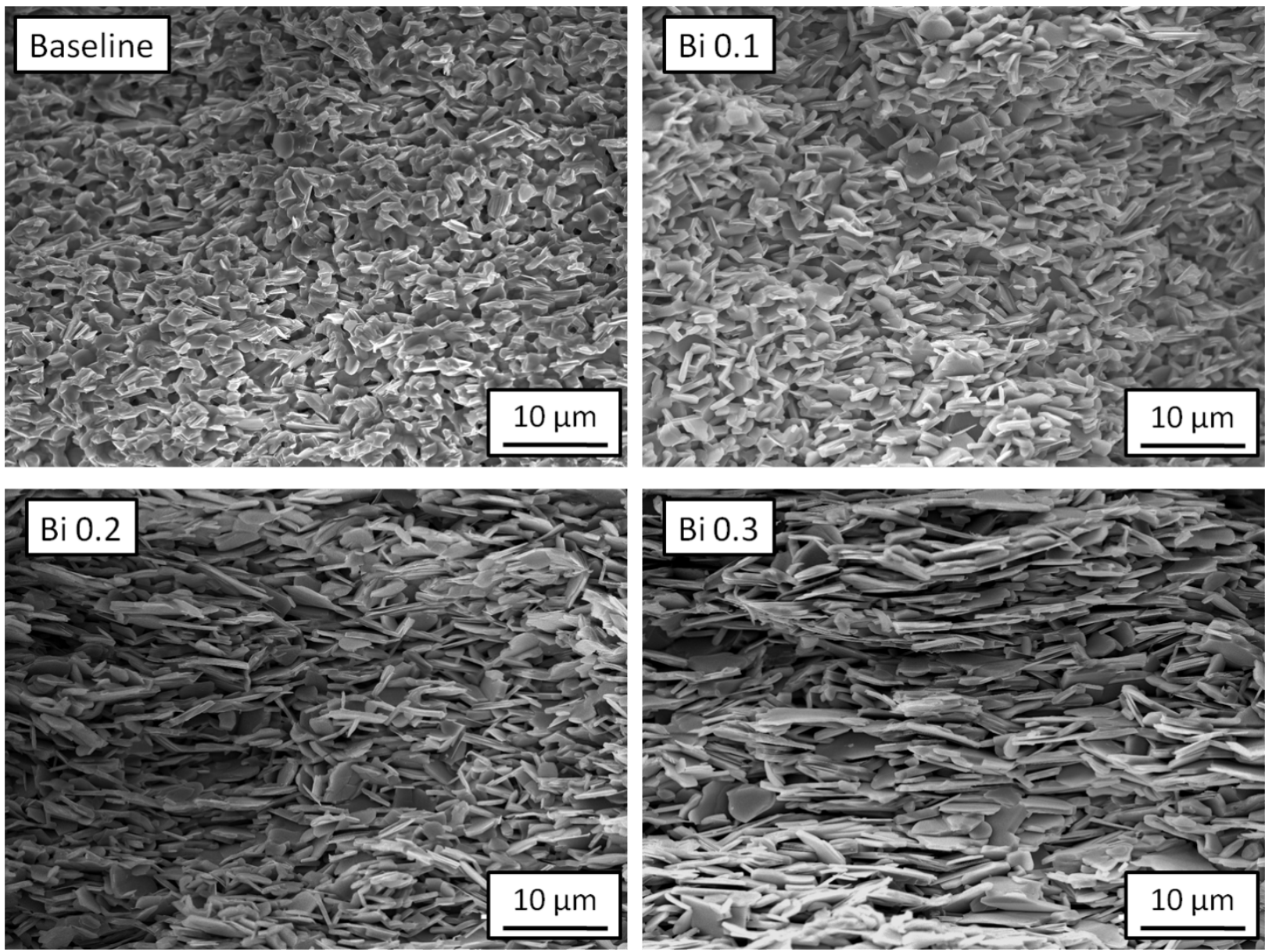

Figure 35. Cross section of baseline and Bi doped CCO pellets. Pressed at $0.5 \mathrm{GPa}$ and $25^{\circ} \mathrm{C}$ for 40 minutes. In plane direction coincides with horizontal direction, perpendicular to pressing axis.

\subsubsection{Transport properties}

The transport properties are highly dependent on the pressing conditions as seen when comparing the graphs for $1 \mathrm{GPa}$ (Figure 26) and 0.5 GPa (Figure 36).The Seebeck coefficient does not show much variation for temperatures below $500 \mathrm{~K}$; above that, the variation begins to increase larger and at $1100 \mathrm{~K}$ the Seebeck coefficient is $40 \mu \mathrm{VK}^{-1}$ larger than that for the $1 \mathrm{GPa}$ pressed set of samples.

The electrical resistivity and the thermal conductivity are also affected with the change in pressing conditions. For the case of the electrical resistivity, an increase of the values with respect to the $1 \mathrm{GPa}$ set of data is observed; this is thought to be related to lower alignment of the grains, also observable from the microstructure images (Figure 33, Figure 34 and Figure 35). For the thermal conductivity 
measurements, the values registered for the new pressing conditions are lower with respect to the $1 \mathrm{GPa}$ set of data. The reduction of the thermal conductivity is thought to be attached to the higher porosity of the specimens for the new pressing setting.

The power factor is reduced for all Bi doping levels with the new pressing conditions. The highest value registered is $0.43 \mathrm{~m} \mathrm{Wm}^{-1} \mathrm{~K}^{-2}$ at $1025 \mathrm{~K}$.
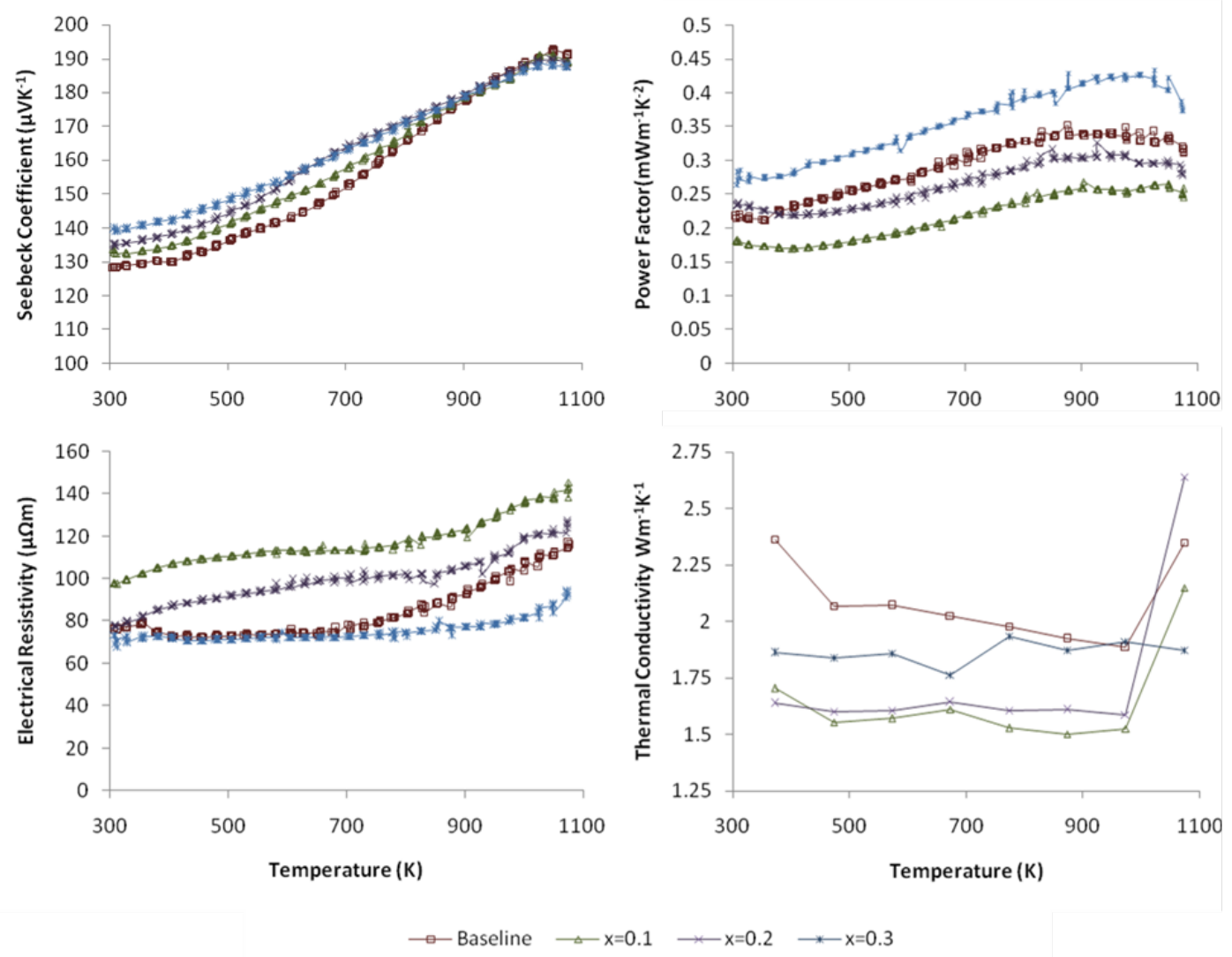

Figure 36. Transport properties for the baseline and Bi doped $\mathrm{CCO}$ samples $\mathrm{x}=0.1,0.2$ and 0.3 . Pressed at $25^{\circ} \mathrm{C}$ and $0.5 \mathrm{GPa}$ for $\mathbf{4 0}$ minutes.

The overall thermoelectric performance seems to be improved with these lower pressure and temperature forming of the samples. Although the power factor is smaller for the $0.5 \mathrm{GPa}$ and $25^{\circ} \mathrm{C}$ pressing parameters, the reduction of the thermal conductivity leads to higher ZT values. Figure 37 presents the dimensionless figure of merit ZT for the new pressing setting. The maximum ZT value is 
0.22 at $1073 \mathrm{~K}$ and is also obtained for the $\mathrm{Bi} 0.3$ doping level; this corresponds to a $29 \%$ of improvement over the baseline better performance.

This opens a new set of variables such as the pressing conditions to be considered in the $\mathrm{CCO}-\mathrm{Bi}$ system, indicating that potential improvement of the system is still possible.
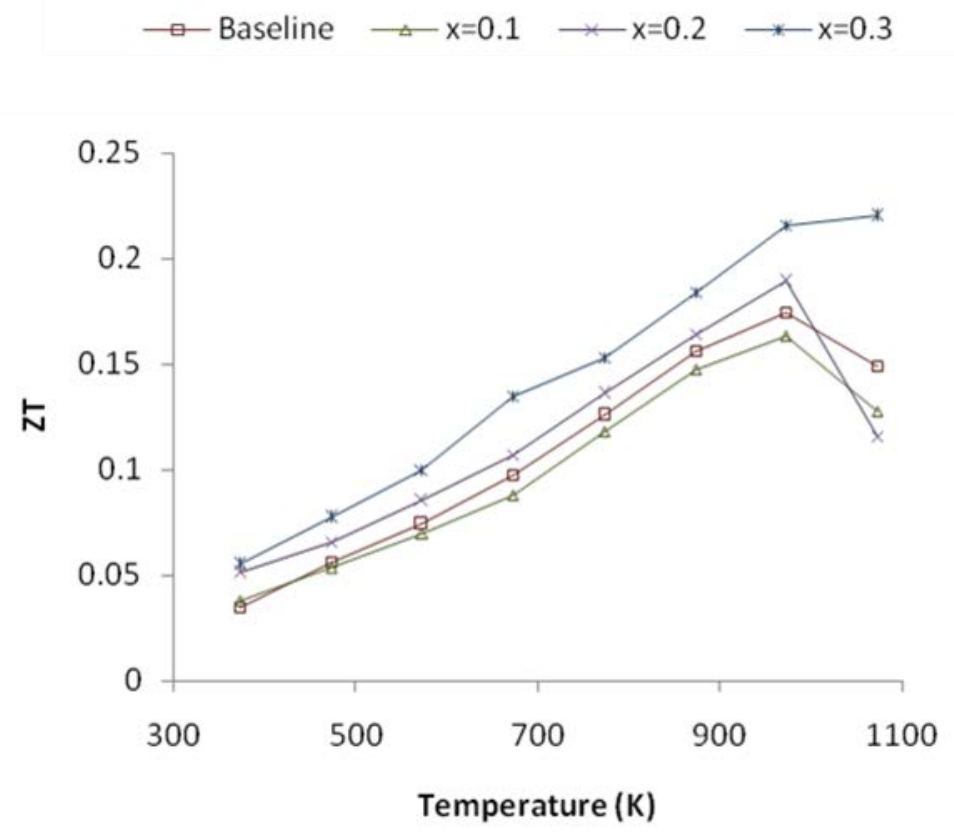

Figure 37. Dimensionless figure of merit for the baseline and Bi doped CCO samples $x=0.1,0.2$ and 0.3 . Pressed at $25^{\circ} \mathrm{C}$ and $0.5 \mathrm{GPa}$ for $\mathbf{4 0}$ minutes. 


\subsection{Conclusions for the Chapter}

The introduction of Bi to CCO increases the density of the compound and seems to trigger grain growth, leading to larger grain sizes as the Bi concentration increases.

$\mathrm{Bi}$ addition up to 0.3 favors the texturing of the polycrystalline samples resulting in reduction of electrical resistivity.

Two types of dislocations between CCO lamellae were identified; in both cases impurity segregation to the crystal defects occur. Higher impurity segregation will occur in grain boundaries of nano-lamellae with different $c$-axes.

$\mathrm{Bi}$ addition to $\mathrm{CCO}$ in 0.3 concentration lead to $33 \%$ improvement for $1 \mathrm{GPa}$ and $150^{\circ} \mathrm{C}$ pressing conditions; for the $0.5 \mathrm{GPa}$ and $25^{\circ} \mathrm{C}$ the improvement is of $22 \%$. These percentages of improvement of ZT are calculated against the corresponding baseline better performances for each of the pressing conditions.

The pressing conditions play a very important role in the final properties of the material. Adequate forming conditions will further enhance the thermoelectric performance of the CCO system. 0.5GPa and $25^{\circ} \mathrm{C}$ pressing conditions prove to be better than $1 \mathrm{GPa}$ and $150^{\circ} \mathrm{C}$ towards higher $\mathrm{ZT}$ values. The thermoelectric performance for the CCO - $0.3 \mathrm{Bi}$ doped samples was improved in $36 \%$ when using the $0.5 \mathrm{GPa}$ and $25^{\circ} \mathrm{C}$ pressing conditions.

Effective doping of $\mathrm{Bi}$ in $\mathrm{CCO}$ for the $\mathrm{Ca}$ site substitution seems to have 0.3 as the concentration limit; $\mathrm{Bi}$ concentrations above this value will diminish the thermoelectric performance of the CCO system, because of electrical resistivity and thermal conductivity increment. 


\section{CHAPTER 4. Effect of Bi Doping on the Ca and Co Sites $\left.\left(\mathrm{Ca}_{(3 \times \times)} \mathrm{Bi}_{(\times+x) y} \mathrm{Co}_{(-4 . y)}\right)_{9}\right)$ on the Thermoelectric Performance and Nanostructure}

Some literatures reported that $\mathrm{Bi}$ in the $\mathrm{CCO}$ system can substitute both atom sites, calcium and cobalt [43]. From the experiments performed, the best Bi doping level for the CCO system is 0.3. A third set of samples was prepared to study the effect of Bi substitution of calcium and cobalt sites simultaneously.

\subsection{Synthesis and Sample Conformation}

The composition formula for this approach is $\mathrm{Ca}_{(3-x)} \mathrm{Bi}_{(x+y)} \mathrm{Co}_{(4-y)} \mathrm{O}_{9}$ with $\mathrm{x}=0.2$ and $\mathrm{y}=0.1$. The powder samples were obtained through the sol-gel route and all the processing parameters were kept identical to those for $\mathrm{Ca}$ site substitution $\mathrm{CCO}-\mathrm{Bi}$ doped system. Two pressing conditions were used for the sample conformation: $0.5 \mathrm{GPa}-25^{\circ} \mathrm{C}$ and $1 \mathrm{GPa}-150^{\circ} \mathrm{C}$. The samples were then sintered and cut to appropriate dimensions for the required measurements.

The precursor powders are shown in Figure 38. The powder sample is similar to the baseline and Ca site $\mathrm{CCO}$ - Bi doped powders. Two kinds of grains can be observed: the platelet like grains are bigger, measuring approximately 500 $\mathrm{nm}$ and the spherical grains are smaller and their size is around $40 \mathrm{~nm}$.

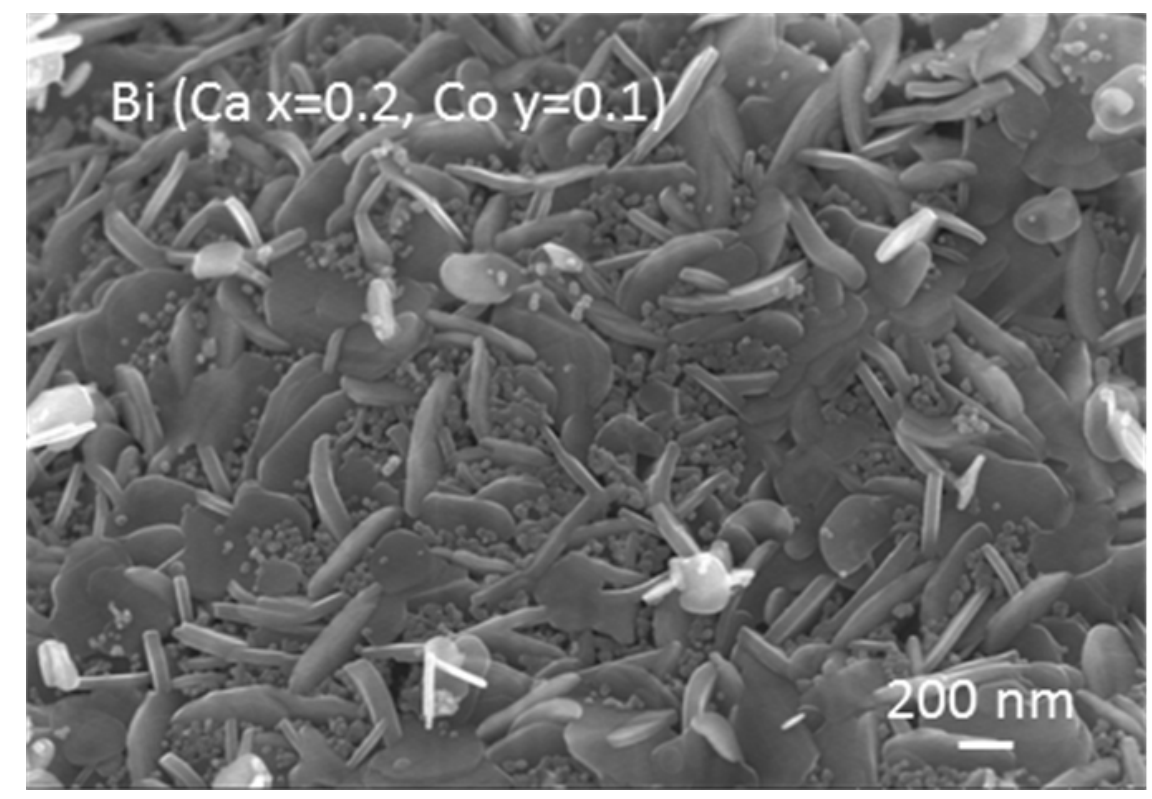

Figure 38. SEM image of CCO - Bi doped on $\mathrm{Ca}$ and $\mathrm{Co}$ sites powders after calcination at $650^{\circ} \mathrm{C}$ for 4 hours. 


\subsection{XRD Measurements}

XRD Characterization of the precursor powders and sintered pellets was performed. The indexed peaks are summarized in Table 7 for the powders. The XRD is taken before the samples are pressed into pellets. All the peaks were consistent with $\mathrm{Ca}_{3} \mathrm{CO}_{4} \mathrm{O}_{9}[13]$ and (PDF 23-110)

Table 7. Powder X-Ray diffraction indexed peaks of CCO Baseline and Bi ( $\mathrm{Ca} X=0.2, \mathrm{Co} y=0.1)$ doped systems.

\begin{tabular}{|c|c|c|c|}
\hline hkl & Sample & 2-Theta position $\left({ }^{\circ}\right)$ & d-spacing (Å) \\
\hline \multirow{2}{*}{001} & Baseline & $*$ & $*$ \\
\hline & $\mathrm{Bi} x=0.2 \mathrm{y}=0.1$ & 8.2864 & 10.6704 \\
\hline \multirow{2}{*}{002} & Baseline & 16.5251 & 5.3646 \\
\hline & $\mathrm{Bi} x=0.2 \mathrm{y}=0.1$ & 16.4222 & 5.3980 \\
\hline \multirow{2}{*}{112 S1 } & Baseline & $*$ & $*$ \\
\hline & $\mathrm{Bi} x=0.2 \mathrm{y}=0.1$ & 33.1557 & 2.7020 \\
\hline \multirow{2}{*}{$20-1$} & Baseline & 37.2662 & 2.4129 \\
\hline & $\mathrm{Bi} x=0.2 \mathrm{y}=0.1$ & 37.2568 & 2.4135 \\
\hline \multirow{2}{*}{$\begin{array}{c}020 \text { S1 } \\
\text { or } \\
11-2 \text { S2 }\end{array}$} & Baseline & 39.5237 & 2.2801 \\
\hline & $\mathrm{Bi} x=0.2 \mathrm{y}=0.1$ & 39.3243 & 2.2912 \\
\hline \multirow{2}{*}{202} & Baseline & 43.6166 & 2.0752 \\
\hline & $\mathrm{Bi} x=0.2 \mathrm{y}=0.1$ & 43.3179 & 2.0888 \\
\hline \multirow{2}{*}{203} & Baseline & $*$ & $*$ \\
\hline & $\mathrm{Bi} x=0.2 \mathrm{y}=0.1$ & 48.4352 & 1.8794 \\
\hline \multirow{2}{*}{220} & Baseline & 55.5662 & 1.6526 \\
\hline & $\mathrm{Bi} x=0.2 \mathrm{y}=0.1$ & 55.3988 & 1.6585 \\
\hline \multirow{2}{*}{$31-2$} & Baseline & $*$ & $*$ \\
\hline & $\mathrm{Bi} x=0.2 y=0.1$ & 67.1893 & 1.3922 \\
\hline
\end{tabular}


The XRD spectrum for the baseline and pellet samples is shown in Figure 39. The peaks are identified and indexed accordingly with the CCO system [13]. The strong 001 reflections confirm the crystal textured nature of the samples.

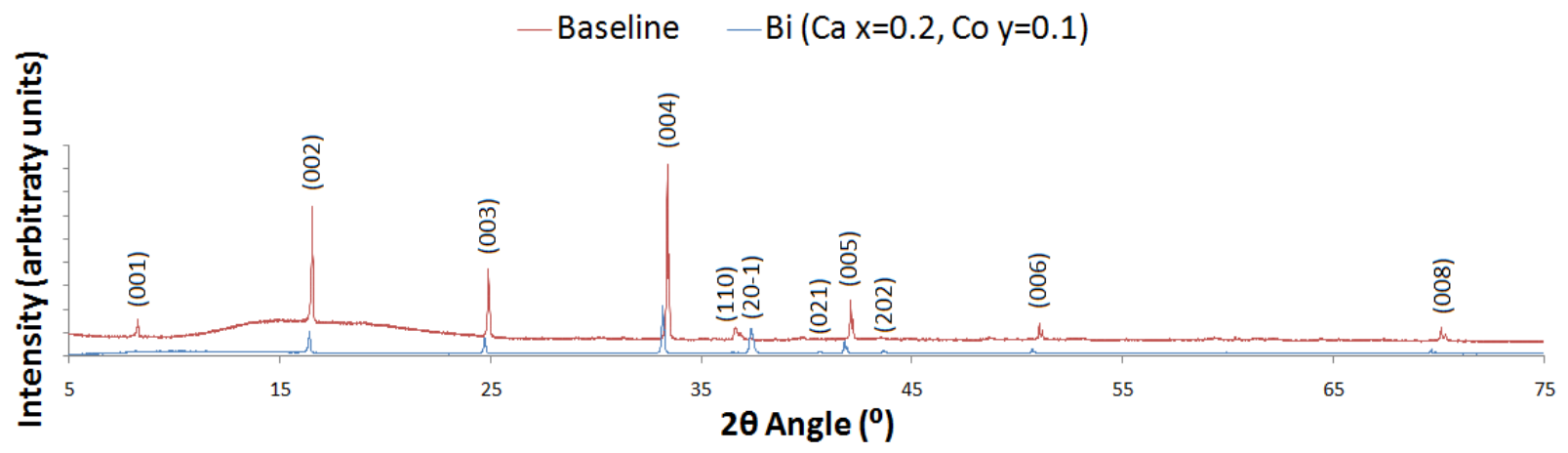

Figure 39. XRD Spectrum of CCO-Bi $x=0.2, y=0.1$ doped system.

\subsection{Density of the Pellets}

The density values obtained for this set of samples shows consistency in of the previous Bi doped system (Ca site only); the $0.5 \mathrm{GPa}$ samples exhibit relative density of $76 \%$ and for the $1 \mathrm{GPa}$ samples of $96 \%$ varying only $2 \%$ and $1 \%$ respectively. Overall, the relative density shows almost no change due to the selective site doping. Table 8 presents the density values and relative densities with respect to the theoretical single crystal density value.

Table 8. Density values for the CCO system, baseline and selective site Bi doped samples pressed at $0.5 \mathrm{GPa}-\mathrm{room}$ temperature and $1 \mathrm{GPa}-150^{\circ} \mathrm{C}$.

\begin{tabular}{|c|c|c|}
\hline Sample & Density $\left(\mathrm{g} / \mathrm{cm}^{3}\right)$ & Relative Density (\%) \\
\hline Single crystal CCO & 4.68 & 100 \\
\hline Baseline $1 \mathrm{GPa}$ & 3.97 & 85 \\
\hline Baseline $0.5 \mathrm{GPa}$ & 3.60 & 77 \\
\hline \multicolumn{3}{|c|}{ Ca $x=0.2$, Co $y=0.1$} \\
\hline $0.5 \mathrm{GPa}$ & 3.57 & 76 \\
\hline $1 \mathrm{GPa}$ & 4.49 & 96 \\
\hline
\end{tabular}




\subsection{SEM imaging}

SEM images of the pressed planes and cross sections of the Ca and Co site Bi doped CCO samples are shown in Figure 40 . All the grains exhibit disc shape. The approximate size is $4 \mu \mathrm{m}$ for the $0.5 \mathrm{GPa}$ and 6 $\mu \mathrm{m}$ for the $1 \mathrm{GPa}$ pressed samples.

The cross section images show uniform and highly crystalline textured samples, in good agreement with the stronger $001 \mathrm{XRD}$ reflections.

The porosity of the samples is evident for both pressing conditions, however it is higher for the lower pressure set of samples; notice that pores are also observed in the pressed plane images. For the higher pressure set of samples higher packing of the grains is observed, along with better alignment of the grains.

These microstructure observations are also confirmed by the density values obtained for the system. 

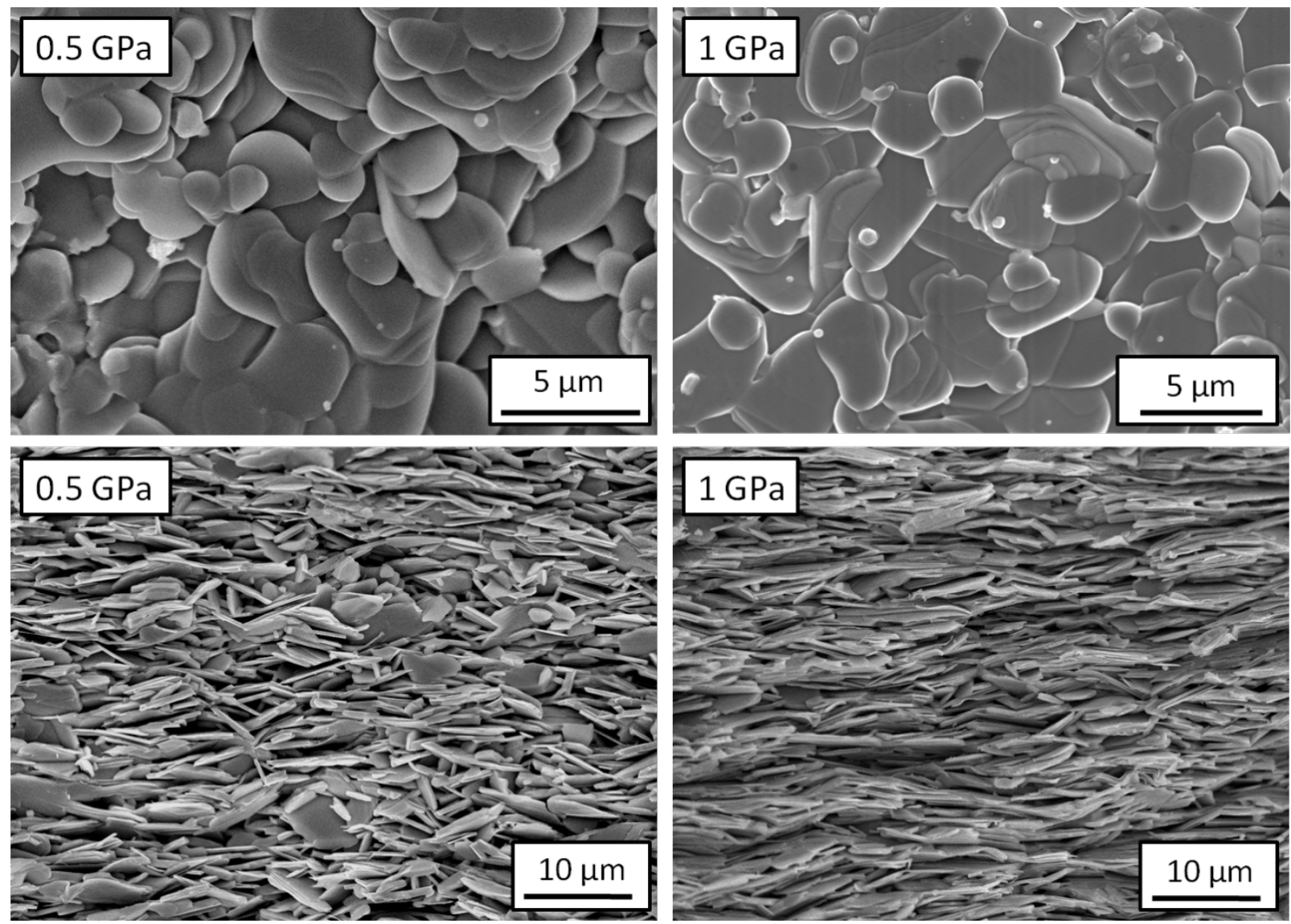

Figure 40. Pressed planes and cross sectional SEM images of CCO - Bi doped Ca $x=0.2$, Co $y=0.1$ levels at 0.5 and $1 \mathrm{GPa}$. For cross section images, in plane direction coincides with horizontal direction, perpendicular to pressing axis.

TEM images of the $0.5 \mathrm{GPa}$ and $25^{\circ} \mathrm{C}$ pressed sample are shown in Figure 41 and Figure 42 . Dislocations along the boundary between lamellae can be observed. The diffraction pattern taken along [110]+[1 $\overline{1} 0]$ is characteristic of CCO. 


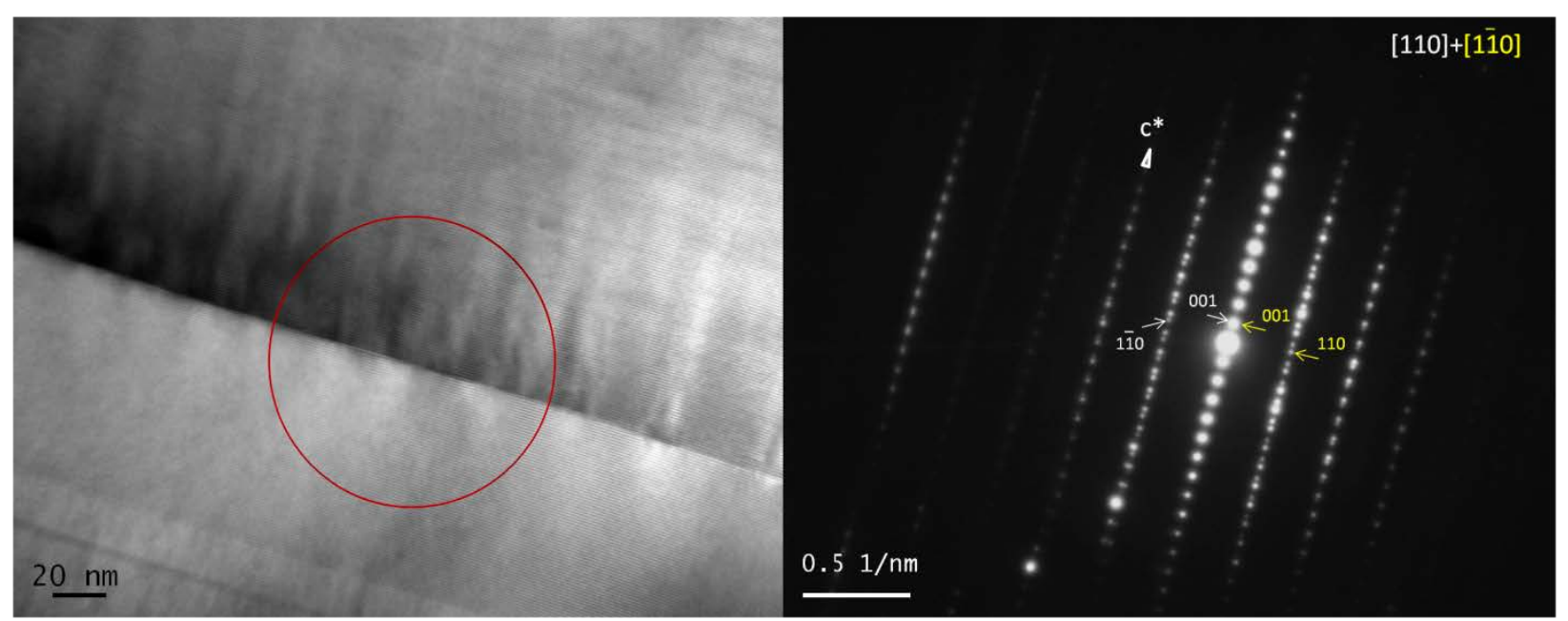

Figure 41. TEM image and diffraction pattern of circled area of CCO - Bi ( $C a x=0.2, y=0.1)$ doped sample pressed at $0.5 \mathrm{GPa}$ and $25^{\circ} \mathrm{C}$.

Figure 42 shows in detail one of the dislocations found in the grain boundary, it can be seen that the nano-lamellae are parallel and at the interface between them rearrangement of the crystal planes occur perturbing adjacent crystal planes propagating towards the interior of the CCO lamella. 


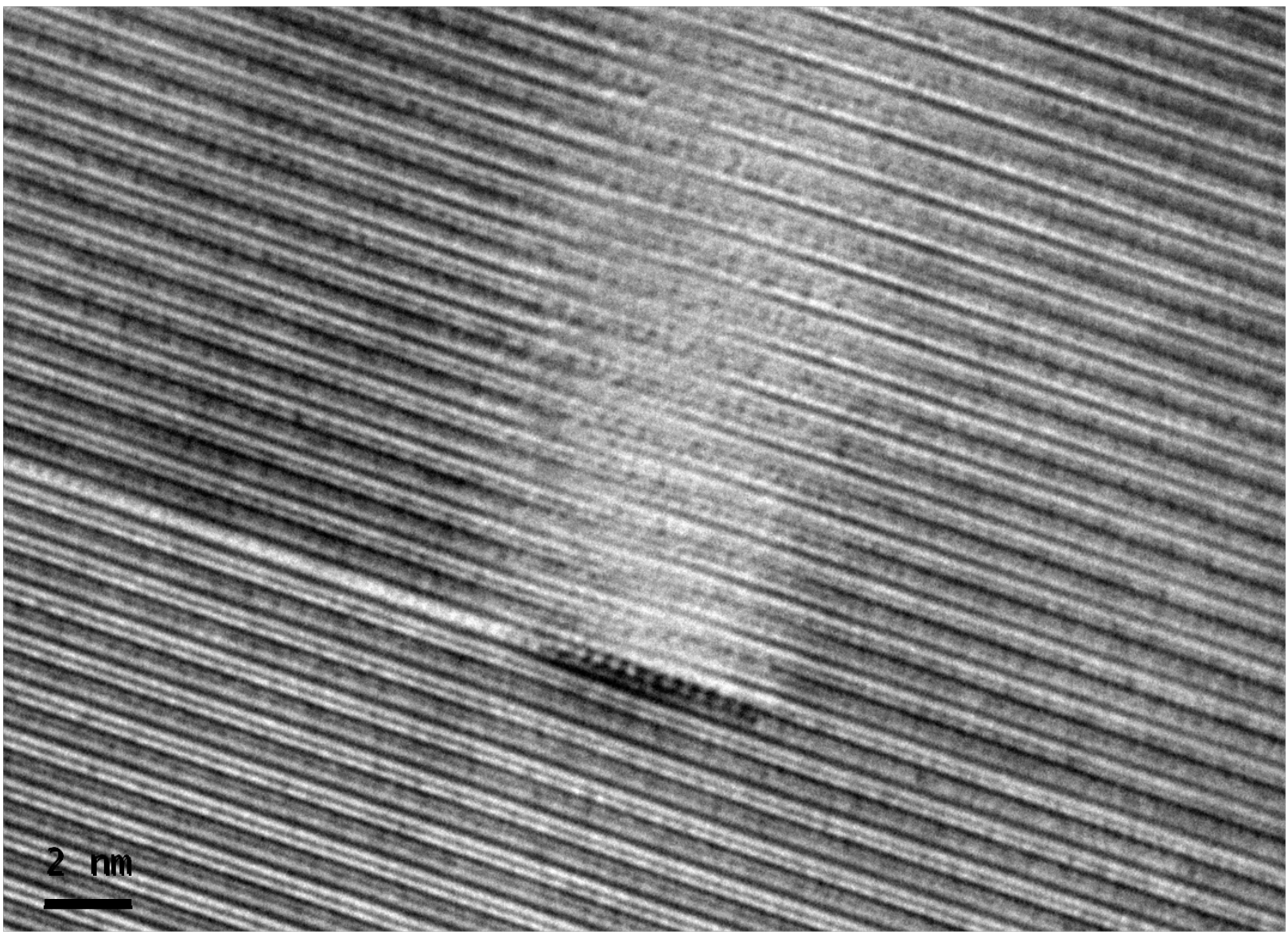

Figure 42. High resolution TEM image of grain boundary Type I with dislocation. $\mathrm{CCO}$ (Ca $\mathrm{x}=0.2, \mathrm{y}=0.1)$ doped sample pressed at $0.5 \mathrm{GPa}$ and $25^{\circ} \mathrm{C}$.

For the samples sintered approximately 15 days after the powder CCO was prepared, needle shape formations similar to those for the Bi 0.4 - Ca site doping levels were observed. This observation suggests that the doping level used for the simultaneous $\mathrm{Ca}$ and Co site substitution doping are not correct. As seen for the Ca site substitution case the optimum level was around 0.3 , however for the Co site 0.1 seems to be excessive. More detailed study of the doping level for the Co site should be carried out. Features are shown in Figure 43 and Figure 44. 

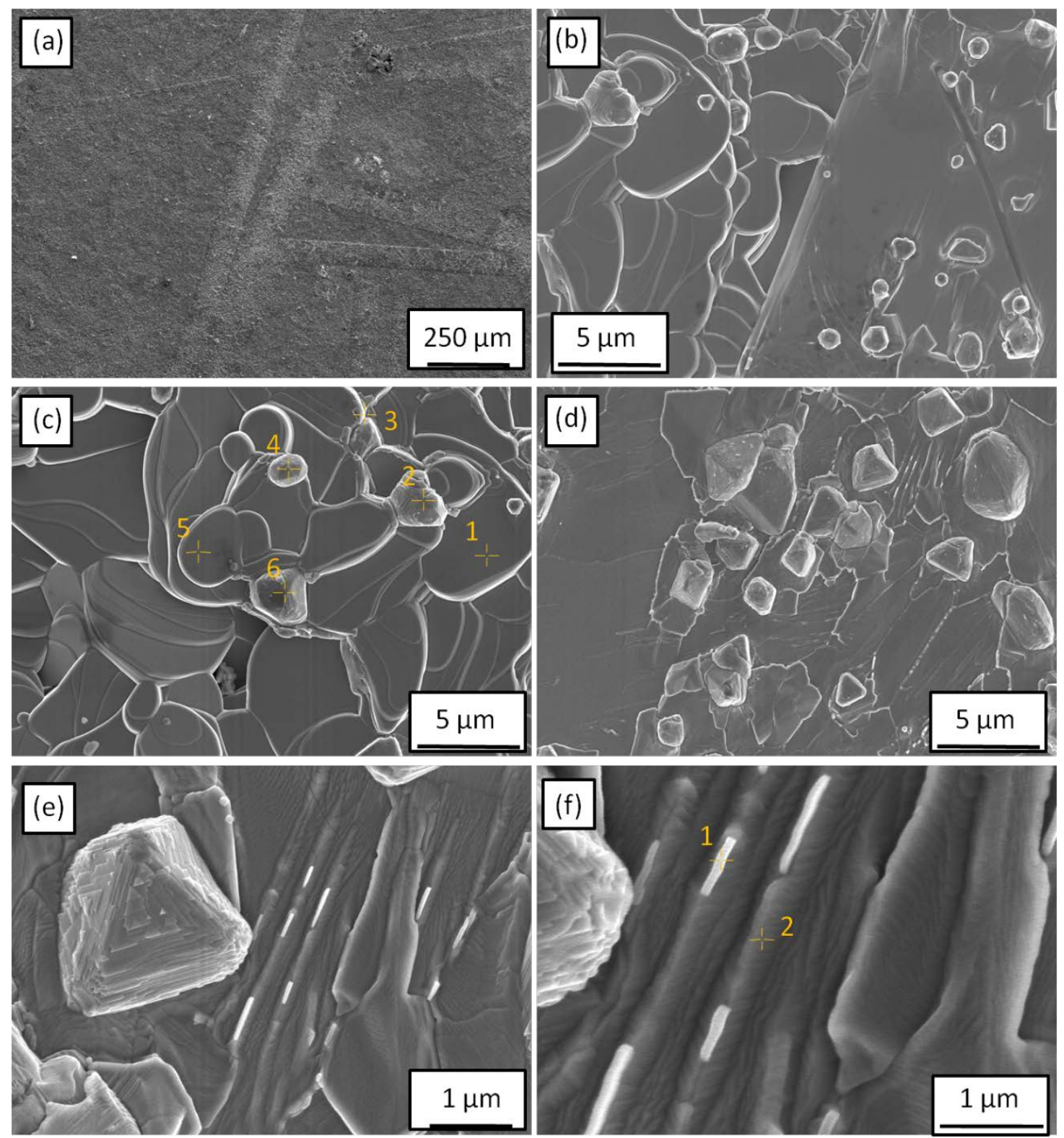

Figure 43. SEM images of the pressed plane for a sample pressed at $1 \mathrm{GPa}$ and $150^{\circ} \mathrm{C}$. Differences in the microstructure features are identified. 

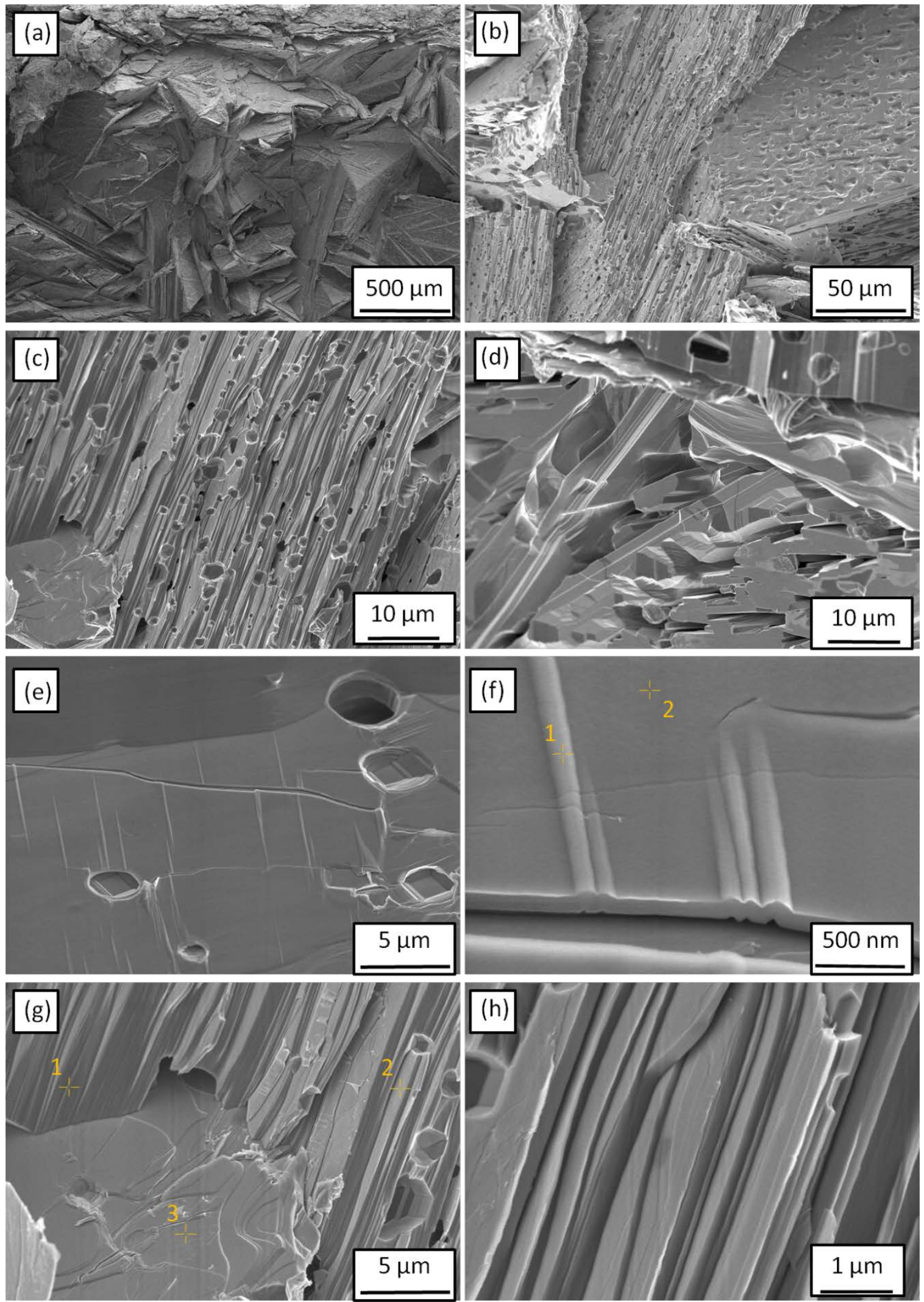

Figure 44. SEM images of the cross section for a sample pressed at $1 \mathrm{GPa}$ and $150^{\circ} \mathrm{C}$. Differences in the microstructure features are identified. 
The EDS analysis reveals that there is a secondary phase of the system. In Figure 43 images (b) to (f) show faceted-triangular shaped grains embedded in the CCO - Bi matrix. These grains are identified as EDS 2, 4 and 6 in image (c); the EDS detailed quantum results is shown in Table 9, from the atomic percentages it is evident that such grains are $\mathrm{Ca}$ and $\mathrm{Bi}$ deficient when compared to the matrix, the phase can be identified as cobalt oxide with $\mathrm{Ca}$ and $\mathrm{Bi}$ doping. This particular composition suggests that Bi substitution in the CCO system occurs for the Ca site preferably. From Figure 44, the homogeneity of the distribution of such grains into the matrix can be observed. The pore-like gaps in images (b), (c), (e), and $(\mathrm{g})$ are the places where the secondary phase grains are located; when the samples are fractured for imaging purposes those grains fell leaving their shape marked. The faceted print left can be more clearly appreciated in image (e) pores.

Table 9. EDS analysis of $\mathrm{CCO}-\mathrm{Bi}(\mathrm{Ca} \mathrm{x}=0.2, \mathrm{y}=0.1)$ 1GPa sample with different microstructure features as depicted in Figure 43 and Figure 44.

\begin{tabular}{|c|c|c|c|c|c|}
\hline \multirow[b]{2}{*}{ Image } & \multirow[b]{2}{*}{ EDS } & \multicolumn{4}{|c|}{ Atomic \% } \\
\hline & & $\mathrm{Ca}$ & Co & 0 & $\mathrm{Bi}$ \\
\hline \multirow{6}{*}{$\begin{array}{l}\text { Pressed plane } \\
\text { (c) }\end{array}$} & 1 & 17.02 & 17.08 & 63.28 & 2.62 \\
\hline & 2 & 1.14 & 25.95 & 72.67 & 0.25 \\
\hline & 3 & 17.19 & 18.63 & 61.57 & 2.61 \\
\hline & 4 & 2.87 & 22.84 & 73.69 & 0.59 \\
\hline & 5 & 18.98 & 19.06 & 59.04 & 2.95 \\
\hline & 6 & 1.83 & 29.59 & 68.22 & 0.36 \\
\hline \multirow{2}{*}{$\begin{array}{l}\text { Pressed plane } \\
\text { (f) }\end{array}$} & 1 & 7.46 & 18.78 & 72.62 & 1.14 \\
\hline & 2 & 8.98 & 20.99 & 68.75 & 1.28 \\
\hline \multirow{2}{*}{$\begin{array}{l}\text { Cross section } \\
\text { (f) }\end{array}$} & 1 & 11.13 & 10.94 & 76.25 & 1.68 \\
\hline & 2 & 11.27 & 11.3 & 75.7 & 1.73 \\
\hline \multirow{3}{*}{$\begin{array}{c}\text { Cross section } \\
\text { (g) }\end{array}$} & 1 & 14.1 & 13.92 & 69.71 & 2.27 \\
\hline & 2 & 13.16 & 12.85 & 72.10 & 1.89 \\
\hline & 3 & 11.09 & 11.03 & 76.25 & 1.63 \\
\hline
\end{tabular}




\subsection{Transport Properties}

The transport properties and ZT values for the simultaneously substituted $\mathrm{Ca}$ and $\mathrm{Co}$ - Bi doped samples are shown in Figure 45 and Figure 46. In the graphs, the blue-square shape represents the $0.5 \mathrm{GPa}-$ $25^{\circ} \mathrm{C}$ pressed samples and the red-diamond shape represents the $1 \mathrm{GPa}-150^{\circ} \mathrm{C}$ pressed samples.

The maximum Seebeck coefficient value is found to be $188.76 \mu \mathrm{VK}^{-1}$ at $1050 \mathrm{~K}$. This value is obtained for the $0.5 \mathrm{GPa}$ and $25^{\circ} \mathrm{C}$ pressed sample. In general the electrical resistivity shows only slight temperature dependence. The minimum electrical resistivity is $47.7 \mu \Omega \mathrm{m}$ at $481 \mathrm{~K}$, corresponding to the sample with $0.5 \mathrm{GPa}$ and $25^{\circ} \mathrm{C}$ pressing conditions; it is worthwhile to point out that low electrical resistivity values around $50 \mu \Omega m$ were obtained for the samples regardless of their pressing conditions.

The thermal conductivity is reduced for the samples pressed at $0.5 \mathrm{GPa}$ and $25^{\circ} \mathrm{C}$ as observed from the thermal conductivity graph in Figure 45 . This result agrees with those from the previous section: 4.2 Effect of Pressing Conditions on Bi doped samples; thermal conductivity is reduced in half by using the $0.5 \mathrm{GPa}$ pressing setting for this particular composition.
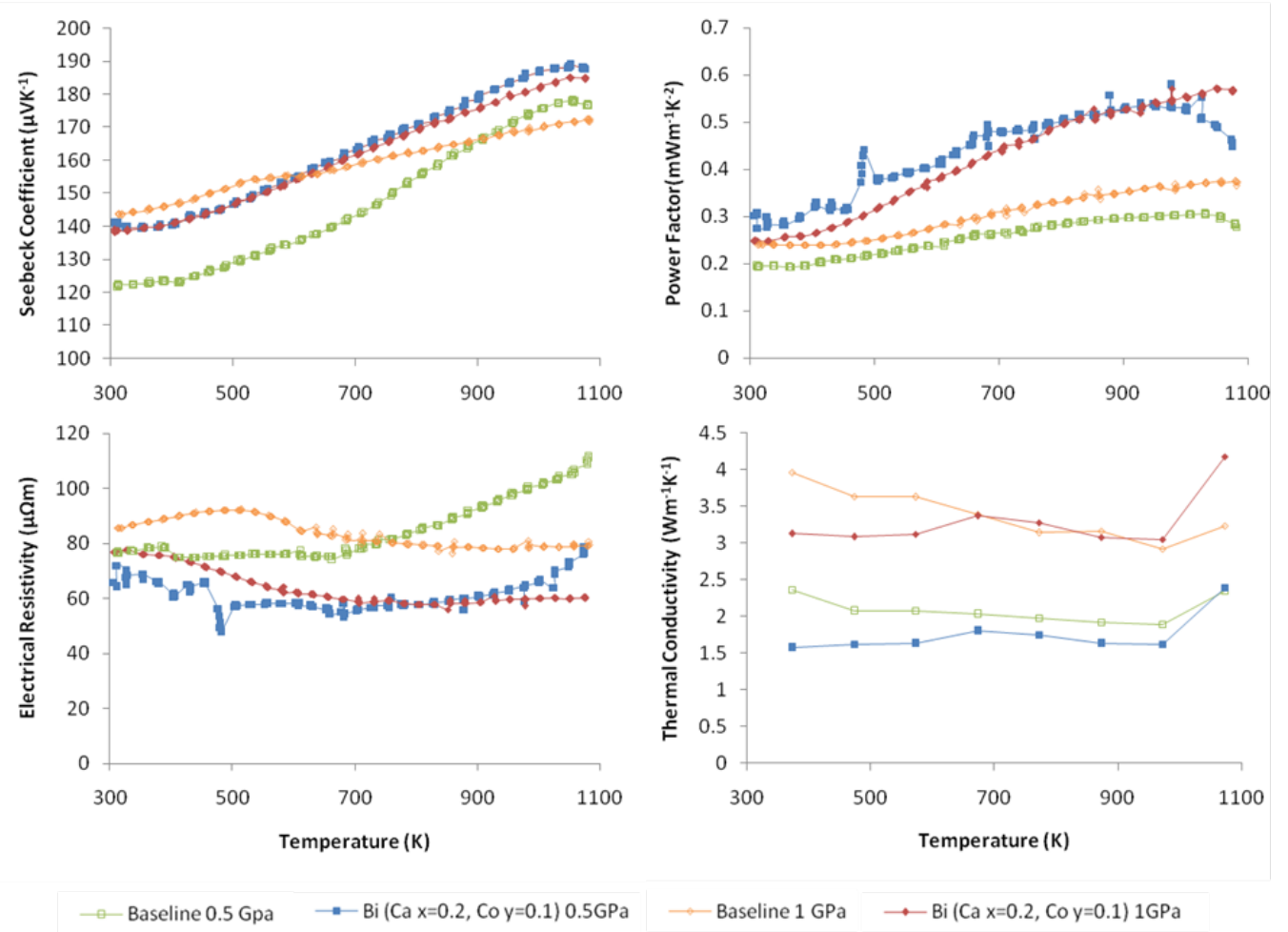

Figure 45. Transport properties for the $\mathrm{Ca}$ and $\mathrm{Co}$ site $\mathrm{Bi}$ doped $\mathrm{CCO}$ samples pressed at $0.5 \mathrm{GPa}-25^{\circ} \mathrm{C}$ and $1 \mathrm{GPa}-150^{\circ} \mathrm{C}$. 
For this $\mathrm{Bi}$ doping composition, the thermoelectric performance represented by the figure of merit ZT reaches a maximum value of 0.32 at $973 \mathrm{~K}$ and is obtained for the sample pressed at $0.5 \mathrm{GPa}$ and $25^{\circ} \mathrm{C}$; corresponding to an improvement of $113 \%$ over the baseline value for this pressing setting. Notice that for both pressing conditions the addition of $\mathrm{Bi}$ as dopant results in enhancement of the thermoelectric performance.
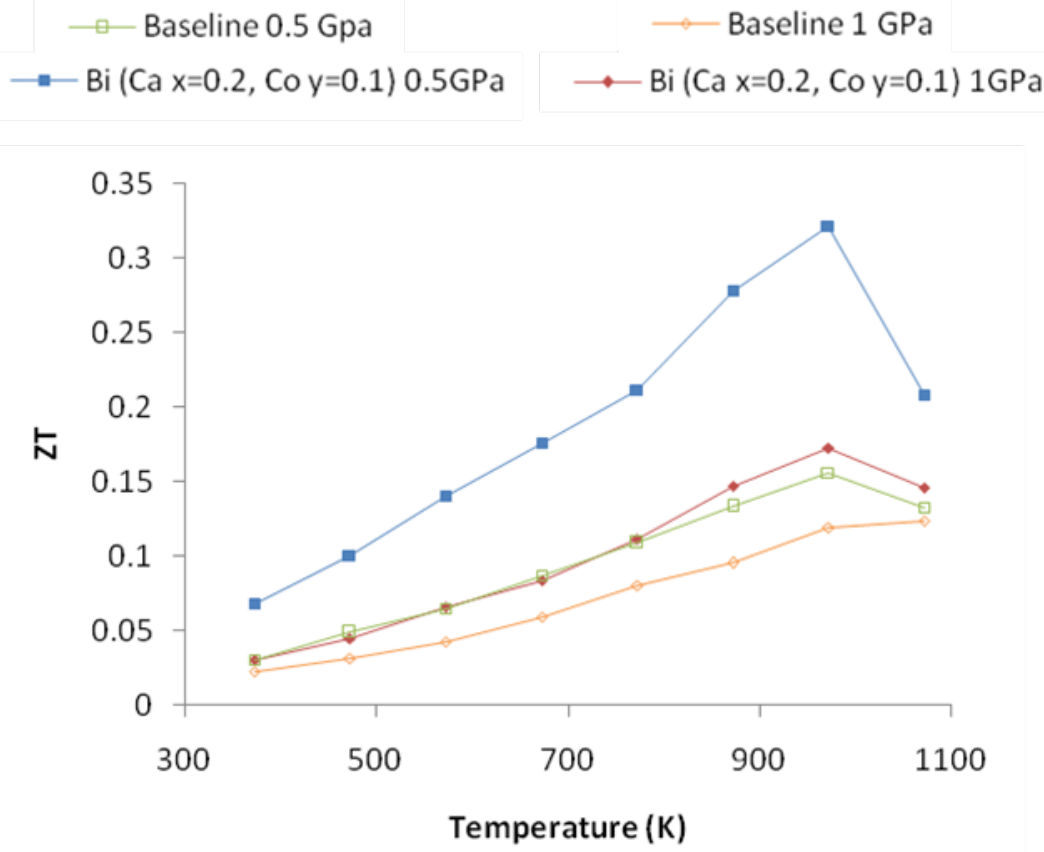

Figure 46. Dimensionless figure of merit for the $\mathrm{Ca}$ and $\mathrm{Co}$ site $\mathrm{Bi}$ doped $\mathrm{CCO}$ samples pressed at $0.5 \mathrm{GPa}-25^{\circ} \mathrm{C}$ and $1 \mathrm{GPa}-$ $150^{\circ} \mathrm{C}$. 


\subsection{Conclusions for the chapter}

The crystalline textured characteristics of the samples were confirmed from the SEM micrographs and XRD spectrums. Higher packing and better alignment is obtained for the $1 \mathrm{GPa}$ and $150^{\circ} \mathrm{C}$ pressing conditions.

Dislocations at the boundaries between CCO lamellae were also observed as for the Ca-site doping case.

Microstructure features have been identified as evidence of the instability of the obtained compound with the formula $\mathrm{Ca}_{(3-x)} \mathrm{Bi}_{(x+y)} \mathrm{Co}_{(4-y)} \mathrm{O}_{9}$ with $\mathrm{x}=0.2$ and $\mathrm{y}=0.1$. Secondary phase grains identified as cobalt oxide were found evidencing the Bi preferred substitution of $\mathrm{Ca}$ atoms in the CCO system.

The Bi limit doping levels for the simultaneous $\mathrm{Ca}$ and $\mathrm{Co}$ substitution have not been studied. Study and refinement of the Bi doping limit for the cobalt site is proposed.

The ZT values obtained for two of these samples were the highest among all other Bi doping concentrations studied in this research. The sample exhibiting the highest ZT value of 0.32 at $973 \mathrm{~K}$ was pressed at $0.5 \mathrm{GPa}$ and $25^{\circ} \mathrm{C}$. 


\section{CHAPTER 5. Effect of Au Nanoinclusions on the Thermoelectric Performance and Nanostructure of $\mathrm{Ca}_{3} \mathrm{Co}_{4} \mathrm{O}_{9}$}

The transport properties of the CCO system are thought to be suitable to enhancement through the addition of metallic nanoinclusions; these are expected to act as phonon scattering elements which will help to reduce the thermal conductivity associated to phonon propagation in the lattice. The electrical transport properties are also improved with metallic particle addition. In the material, the metallic particles act as electrical contacts between the cobaltite grains reducing the electrical resistivity.[47]

Care must be paid to the amount of metallic phase as well as its dispersion into the material because agglomeration and large particle size (greater than or comparable to the CCO grains) can short-circuit the carrier flow, i. e. they will act as electrical bypasses nullifying the thermoelectric voltage.[47, 49]

\subsection{Synthesis and Sample Conformation}

CCO plain powders were prepared by the sol-gel route; hydrothermal deposition of Au 10wt\% was followed, using CCO powders as seed material for Au nanoparticles nucleation. The obtained CCOAu10\% composite was subsequently mixed with plain CCO powders to specific Au concentrations of $1 w t \%, 2 w t \%$ and $5 w t \%$ in order to keep the $A u$ nanoinclusions homogeneity when varying concentration. The powder samples were pressed into pellets and sintered as described previously and cut to appropriate dimensions for testing.

Images of the precursor powders CCO - Au10\% were taken for composition analysis EDS and further understanding of the transition taking place during sintering. In Figure 47 the powders of CCO - Au 10\% composites just before pressing are imaged. The big flakes are the CCO grains and the smaller brighter dots are the Au nanoparticles.

The Au nanoparticles are well dispersed throughout all the CCO powder and almost no agglomeration of Au is observed. 


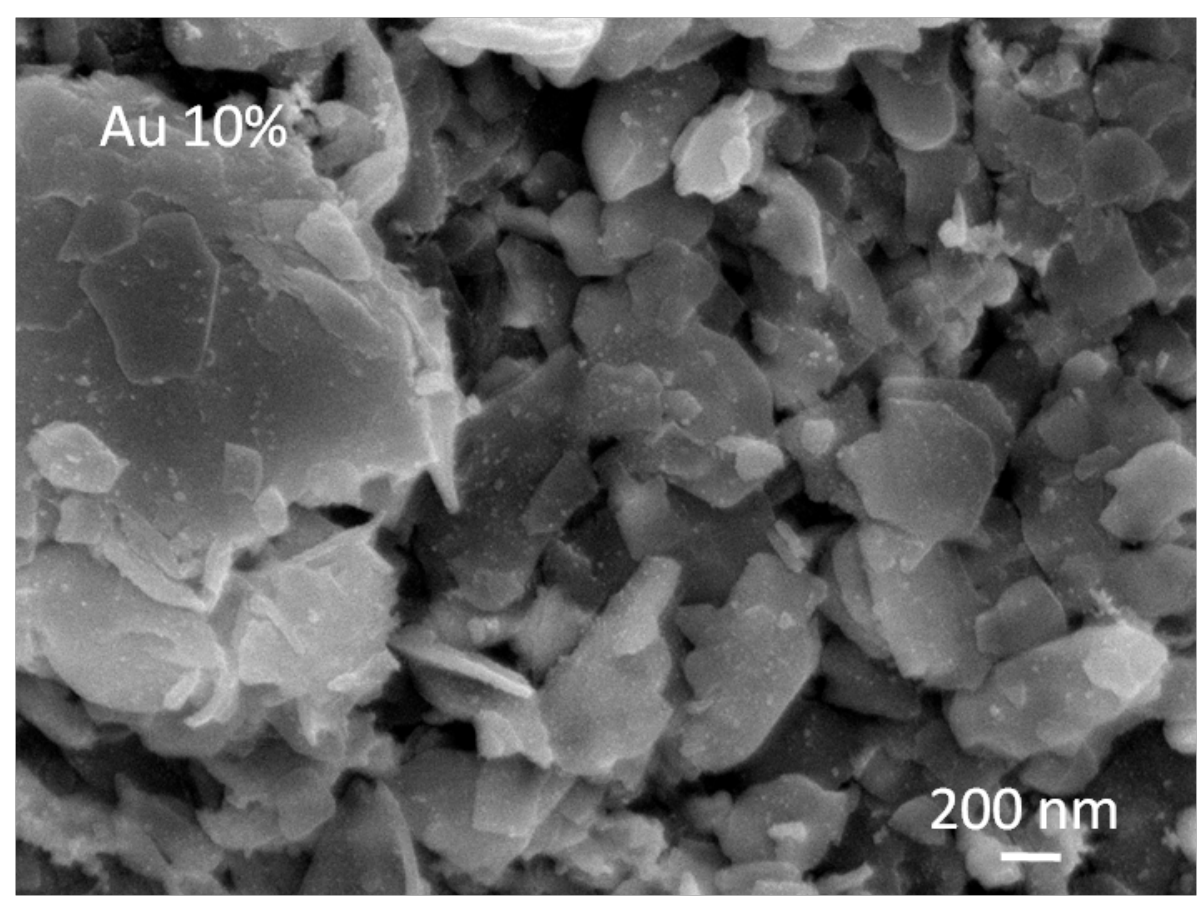

Figure 47. SEM image of CCO - Au $10 \%$ powders after hydrothermal deposition and drying at $300^{\circ} \mathrm{C}$ for 4 hours.

Very fine Au nanoparticles are observed on the surface of the CCO grains (See Figure 48). The size and distribution of the nanoparticles is homogeneous over the entire batch of material prepared. The size of the particles is approximately $2.5 \mathrm{~nm}$ and its shape is presumably spherical since not high enough resolution for such small features was possible to obtain.
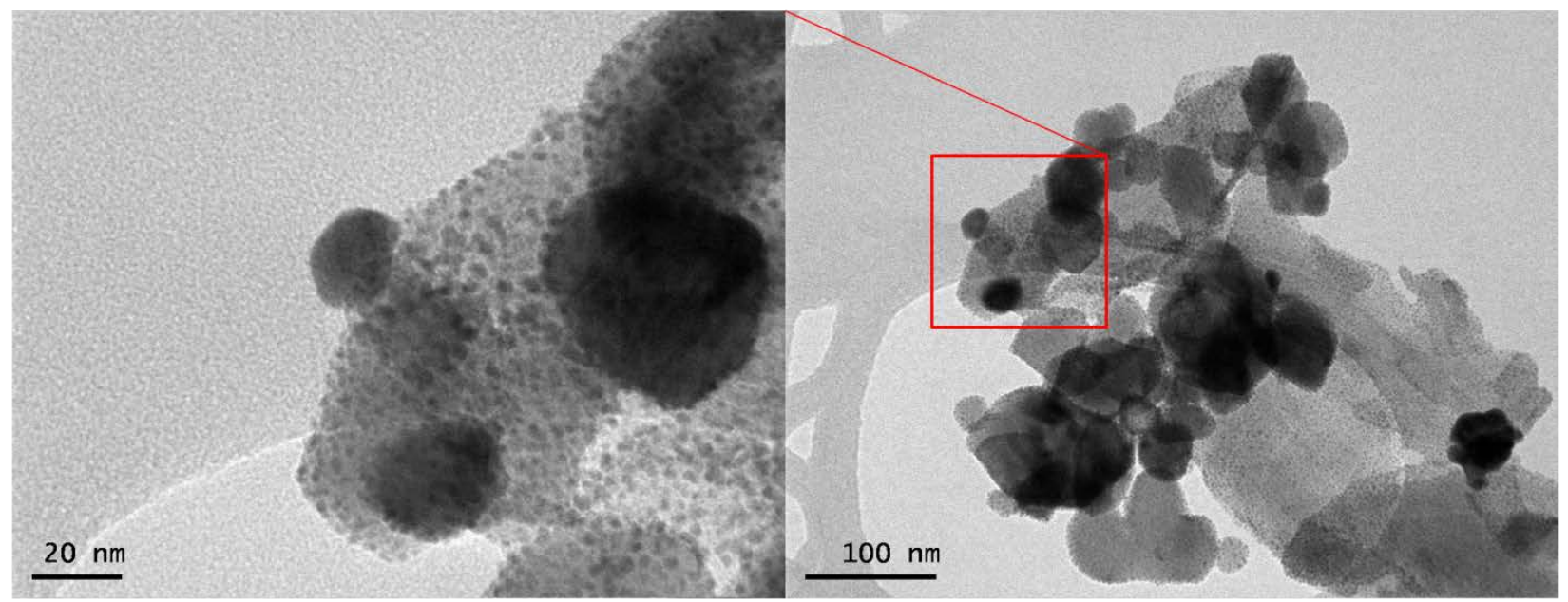

Figure 48. TEM images of the CCO - Au10\% powders after hydrothermal deposition. 
The EDS confirms the CCO - Au composition over the entire batch suggesting that the powders have homogeneous characteristics. In the TEM images the darker small dots (approximately 3 to $5 \mathrm{~nm}$ ) are Au nanoparticles. The calcium and cobalt composition was confirmed to match CCO atomic ratios. Cobalt oxide is also present as in the baseline powder precursors.

\subsection{Density}

The experimental density values for the CCO - Au wt \% composites are summarized in Table 10. There are very small changes in density. For the Au $1 \mathrm{wt} \%$ the density is slightly greater than that for the baseline; however, there is in general decrease of the density with increase of Au\% nanoinclusions. This result agrees with those from Xiang et. al. 2008.

Table 10. Density of CCO systems, baseline and Au composite series.

\begin{tabular}{lccc}
\multicolumn{2}{c}{ Sample } & Density $\left(\mathrm{g} / \mathrm{cm}^{3}\right)$ & Relative Density (\%) \\
\hline $\begin{array}{l}\text { Single crystal CCO } \\
\text { Baseline 1 GPa }\end{array}$ & 4.68 & 100 \\
& wt \% & 3.97 & 85 \\
& 1 & & 86 \\
$\mathrm{Au}$ & 2 & 4.02 & 85 \\
& 5 & 3.98 & 85 \\
& 10 & 3.96 & 83 \\
\hline
\end{tabular}

\subsection{XRD Measurements}

XRD Characterization of the CCO - Au 10wt\% powders and the systematic set of pellet samples was performed and is shown in Table 11 and Figure 49 respectively.

The peaks were indexed as $\mathrm{Ca}_{3} \mathrm{CO}_{4} \mathrm{O}_{9}$ according to[13] and (PDF 23-110). The Au metallic particles were identified from peaks 111, 200 and 311 according to reference (PDF 4-748). 
Table 11. Powder X-Ray diffraction indexed peaks of CCO Baseline and CCO - Au wt \% added systems.

\begin{tabular}{|c|c|c|c|}
\hline Hkl & Sample & 2-Theta position $\left({ }^{\circ}\right)$ & d-spacing $(\AA)$ \\
\hline \multirow{2}{*}{002} & Baseline & 16.5251 & 5.3646 \\
\hline & Au $10 w t \%$ & 16.5761 & 5.3482 \\
\hline \multirow{2}{*}{003} & Baseline & $*$ & $*$ \\
\hline & Au $10 w t \%$ & 24.8645 & 3.5810 \\
\hline \multirow{2}{*}{004} & Baseline & 33.4295 & 2.6805 \\
\hline & Au $10 w t \%$ & 33.3845 & 2.6840 \\
\hline \multirow{2}{*}{$20-1$} & Baseline & 37.2662 & 2.4129 \\
\hline & Au $10 w t \%$ & 37.3048 & 2.4105 \\
\hline $111 \mathrm{Au}$ & Au $10 w t \%$ & 38.1870 & 2.3568 \\
\hline $200 \mathrm{Au}$ & Au $10 w t \%$ & 44.3282 & 2.0435 \\
\hline \multirow{2}{*}{$11-5$} & Baseline & $*$ & $*$ \\
\hline & Au $10 w t \%$ & 55.0896 & 1.6671 \\
\hline \multirow{2}{*}{$11-7$} & Baseline & $*$ & $*$ \\
\hline & Au $10 w t \%$ & 64.5729 & 1.4433 \\
\hline $311 \mathrm{Au}$ & Au $10 w t \%$ & 77.7609 & 1.2272 \\
\hline
\end{tabular}

From the XRD spectrum of the CCO - Au composites it is seen that the samples exhibit preferential orientation and that CCO grains align mostly perpendicular to the c - axis since reflections $00 \mathrm{l}$ predominate.

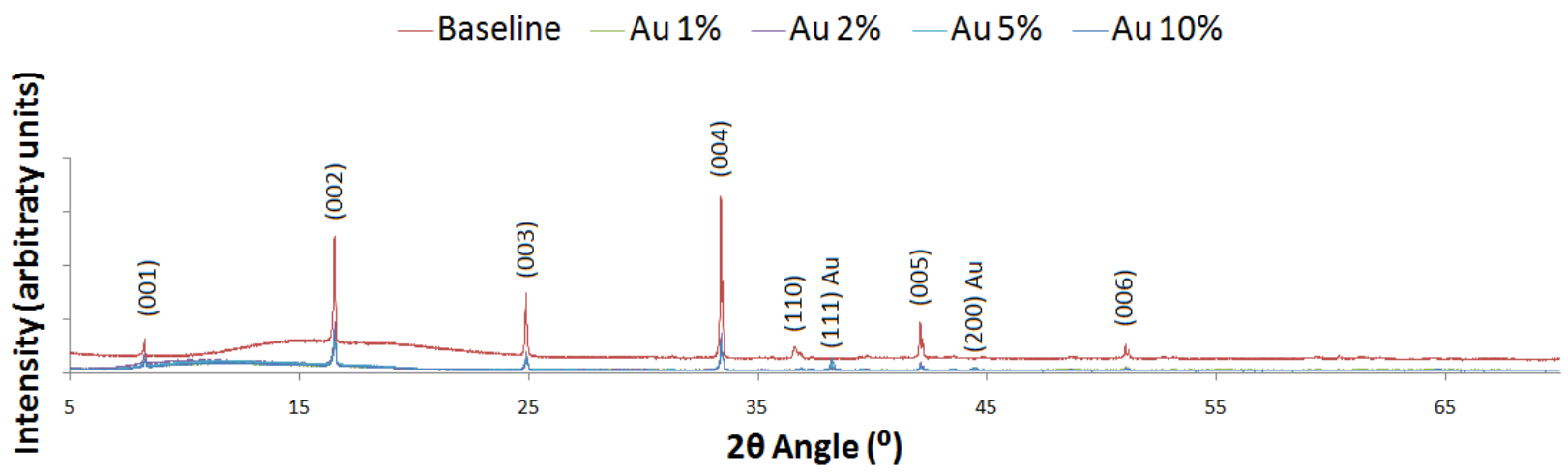

Figure 49. XRD spectrum for baseline and CCO - Au\% samples.Au concentrations of $1 \%, 2 \%, 5 \%$ and $10 \%$. 


\subsection{SEM imaging}

The SEM images of the pressed planes (top view of the pressed surface) and cross sections (perpendicular to the pressure axis) of the samples are shown in (Figure 50, Figure 51, Figure 52) and (Figure 53) respectively. Au nanoparticles appear as smaller brighter dots of $\sim 50 \mathrm{~nm}$ up to $\sim 250 \mathrm{~nm}$. Larger and less bright grains are CCO. Au particles have grown in the sintering process 20 to 100 times greater than their original size when hydrothermally deposited.

In the images, porosity of the polycrystalline samples can be appreciated. The Au nanoparticles are very well dispersed around the sample volume for the $10 \mathrm{wt} \%$ Au composite, less uniformity in this distribution of Au particles is seen in images for lower Au concentrations.

The CCO grain size is $2 \mu \mathrm{m}$ and is approximately constant though all the samples regardless of the Au\% composition.

The $\mathrm{Au}$ nanoparticles experience great growth during the sintering process. The size of the Au nanoparticles in the synthesized powder is just 3 to $5 \mathrm{~nm}$; in contrast for the sintered pellets the Au nanoinclusions can be as high as 250 to $300 \mathrm{~nm}$. (See Figure 50 and Figure 53) 
CHAPTER 5. EFFECT OF AU NANOINCLUSIONS ON THE THERMOELECTRIC PERFORMANCE AND NANOSTRUCTURE OF $\mathrm{Ca}_{3} \mathrm{CO}_{4} \mathrm{O}_{9}$
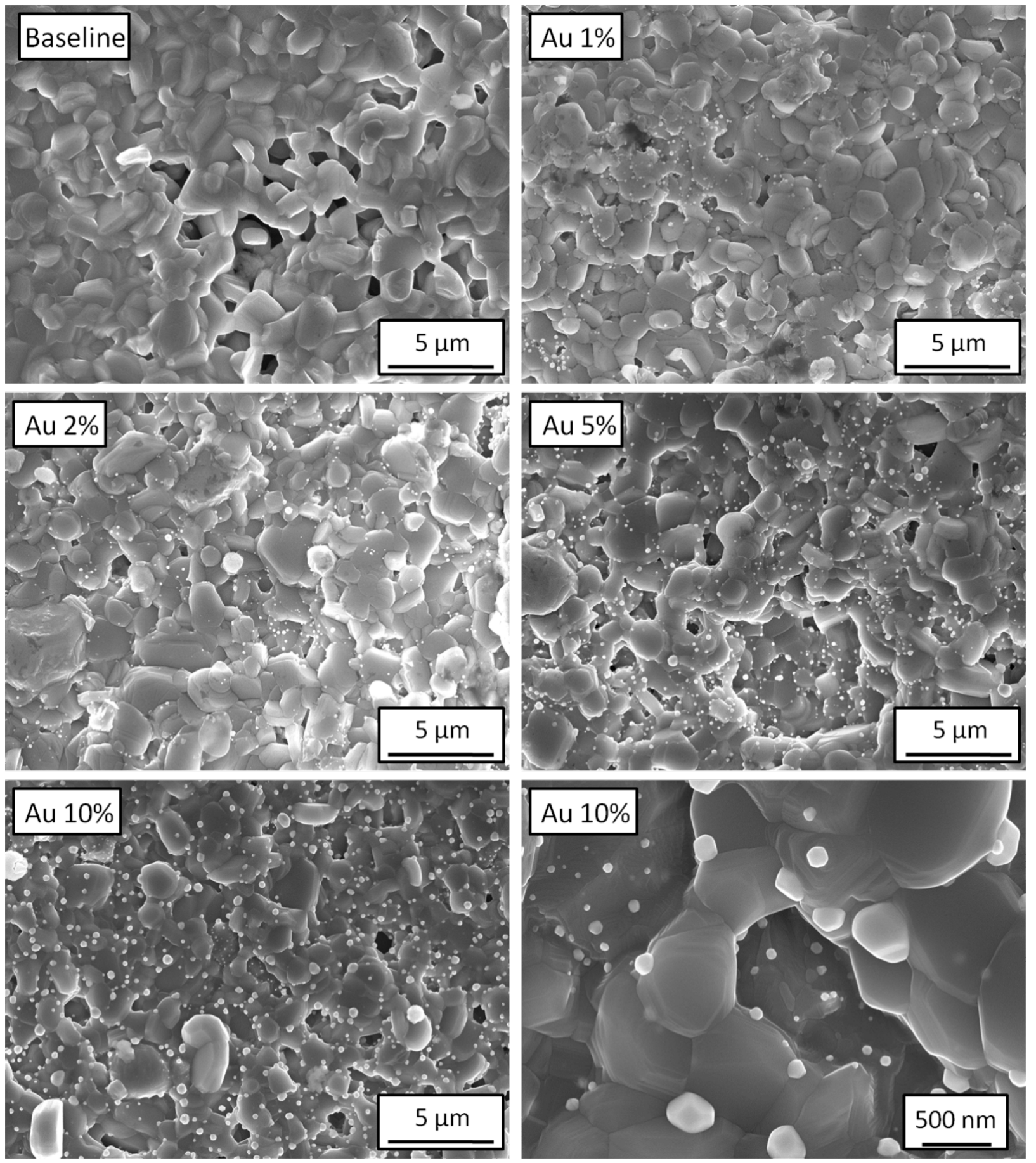

Figure 50. Pressed plane of baseline and Au composite CCO pellets.Pressed at $1 \mathrm{Gpa}$ and $150^{\circ} \mathrm{C}$ for 40 minutes. 

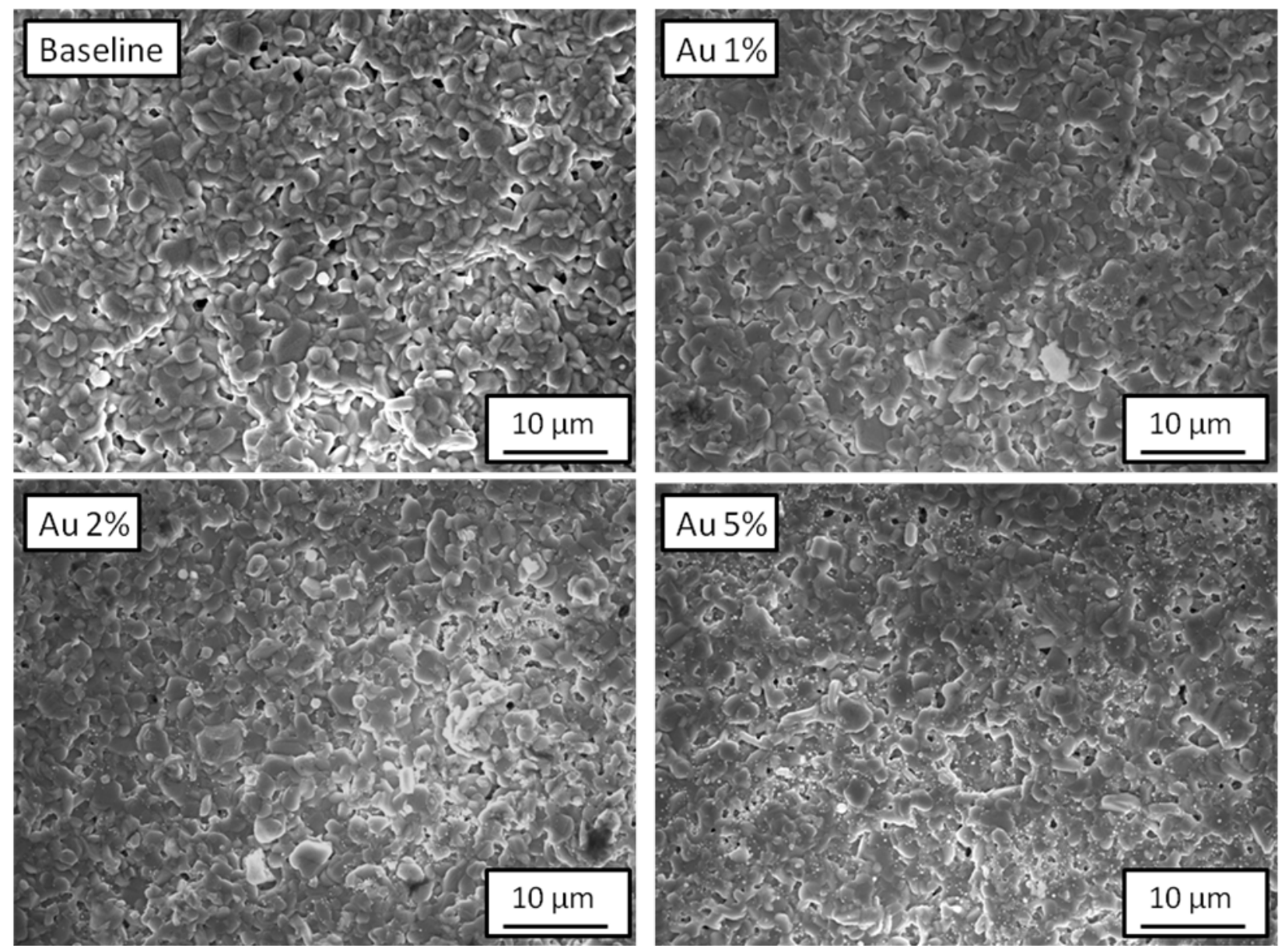

Figure 51. Pressed plane of baseline and CCO - Au nanoinclusionsadded samples pressed at $1 \mathrm{GPa}$ and $150^{\circ} \mathrm{C}$ (lower magnification). 


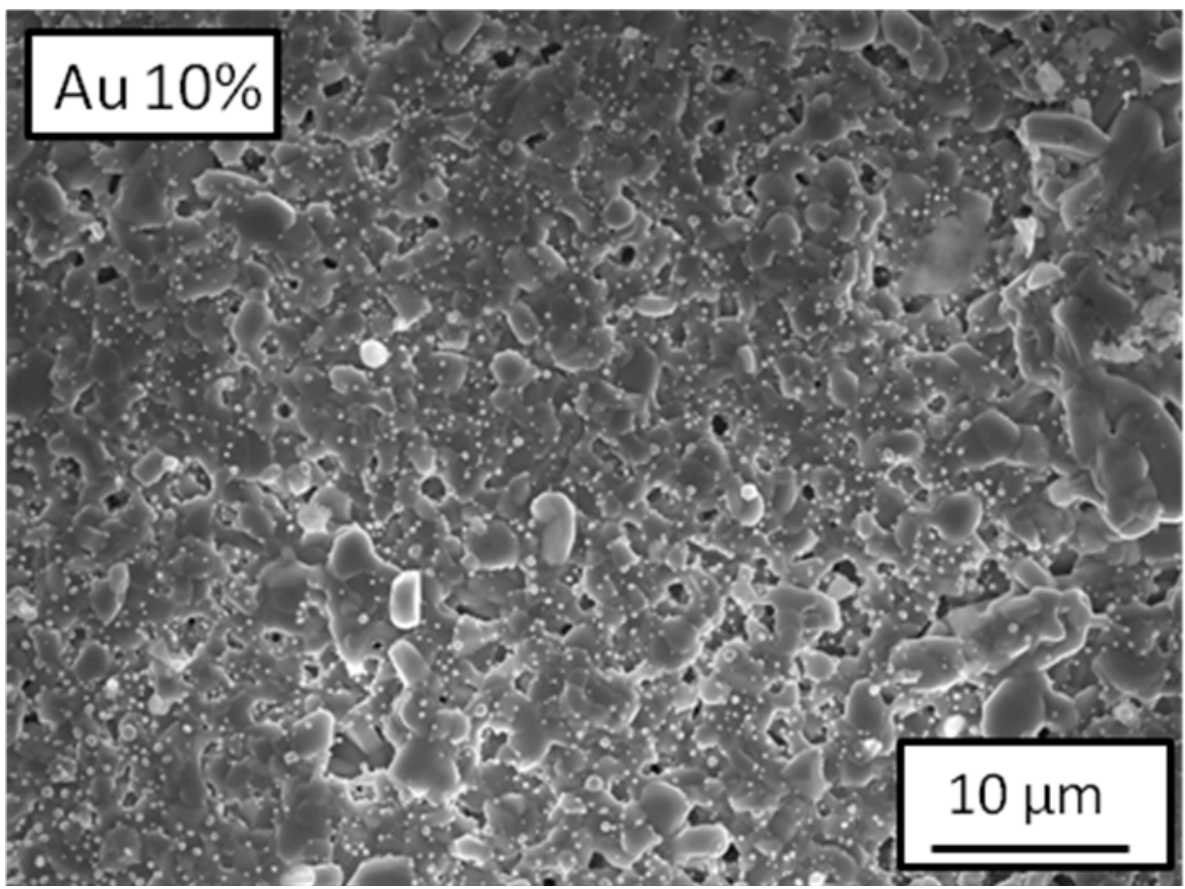

Figure 52. Pressed plane of baseline and CCO - Au $10 \mathrm{wt} \%$ nanoinclusions added samples pressed at $1 \mathrm{GPa}$ and $150^{\circ} \mathrm{C}(\mathrm{lower}$ magnification).

The high porosity and poor alignment of the composites is also confirmed by the density calculations which highlight Au addition as a diminish factor of the relative density. Nevertheless, the poor alignment is also observed for the baseline samples.

There has been reported that Ag nanoinclusions favor the alignment of the polycrystalline CCO samples in concentrations below 7.5 vol\%.[45] However from the SEM images it is observable that no alignment improvement was achieved by introducing Au nanoparticles. This difference might be explained due that the sintering process used in that report involves pressure; whereas in this study no pressure is exerted on the samples while the annealing process takes place. Their hypothesis for alignment improvement is based on the sliding of CCO grains on softened Ag particles when pressure is applied during sintering.

Since there is no improvement on the alignment, low effect on the reduction of electrical resistivity is expected. On the other hand the high porosity is expected to reduce the thermal conductivity; this, in turn could lead to enhancement of the thermoelectric performance. 

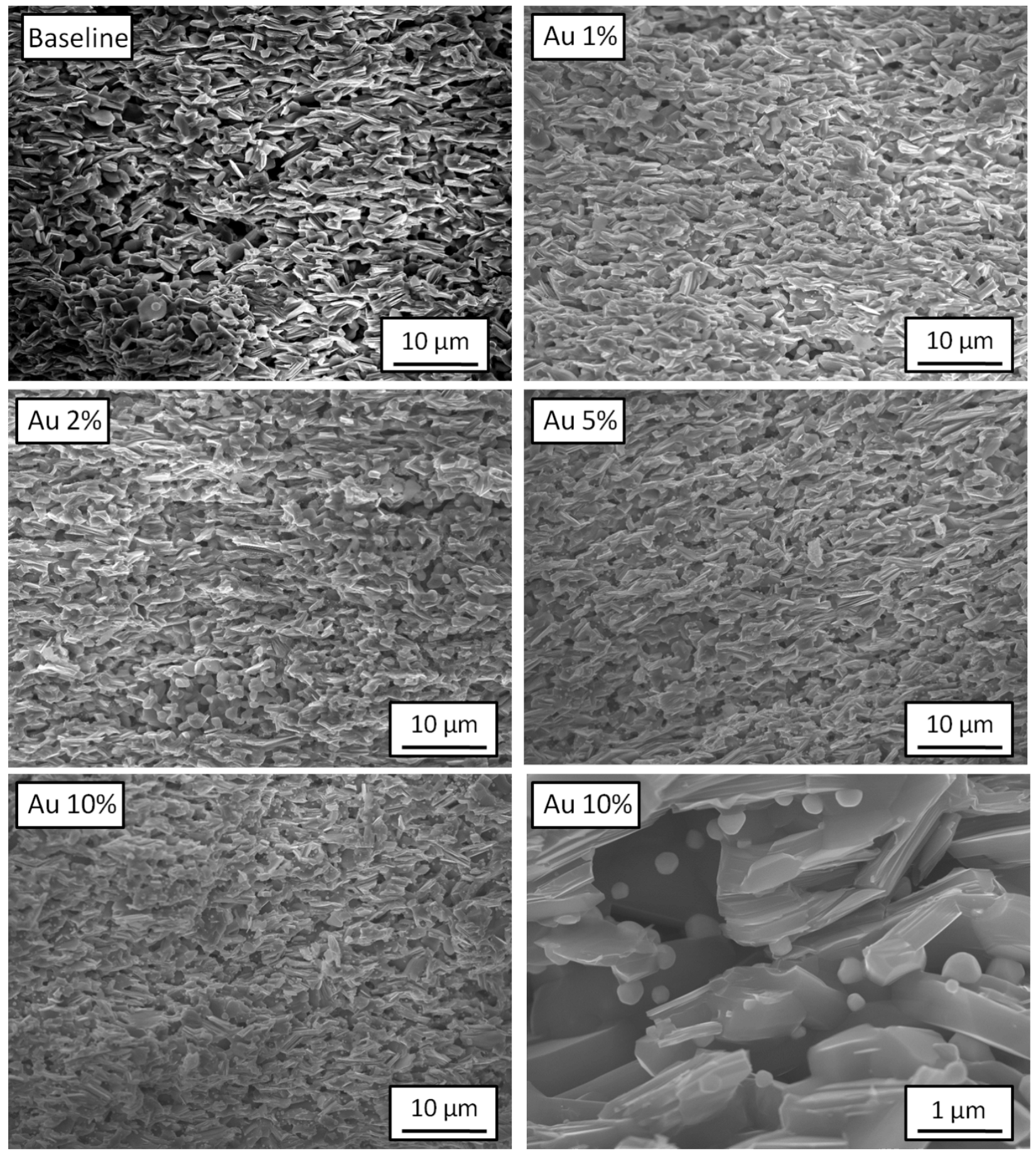

Figure 53. Cross section of baseline and Au composite CCO pellets. Pressed at $1 \mathrm{Gpa}$ and $150^{\circ} \mathrm{C}$ for 40 minutes. In plane direction coincides with horizontal direction, perpendicular to pressing axis. 
In Figure 54 and Figure 55 TEM images of the CCO - Au 1\% samples are shown. In the low magnification images (Figure 54), the dark spots of approximately $50 \mathrm{~nm}$ to $200 \mathrm{~nm}$ are the Au inclusions and the layered matrix is composed by the CCO grains. It can be appreciated that the size of the Au particles is not uniform and that the particles have grown larger than their dimensions once the hydrothermal deposition was finished (Figure 48).

From image (b) in Figure 54, it can be seen that there is alignment of the CCO grains and that the grains are mainly parallel to each other.
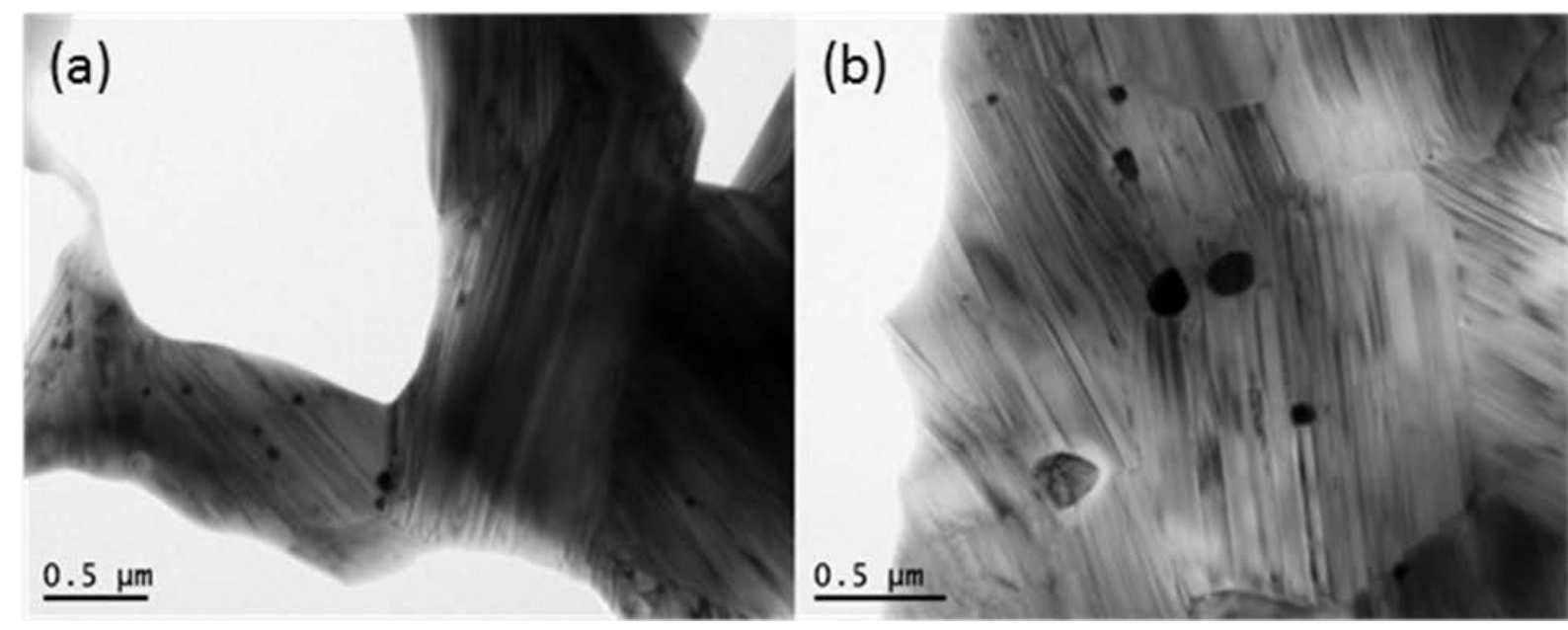

Figure 54. TEM images of the CCO - Au $1 \%$ composite samples.

The Au metallic particles can be found at the interior of the grains as well as in the grain boundaries. From images in Figure 55 high coherence between the CCO planes and the Au planes is observed. Straight edges show the boundaries between the Au particles and the CCO matrix; and the rounded edges also show coherence and alignment between particle and matrix. The diffraction patterns shown (a) and (d) images of Figure 55 are characteristic of the CCO system. Notice that reflections from the CCO system as well as from the Au metallic particles is observed in image (d); here the Au spots appear forming lines perpendicular to those attributed to CCO. 


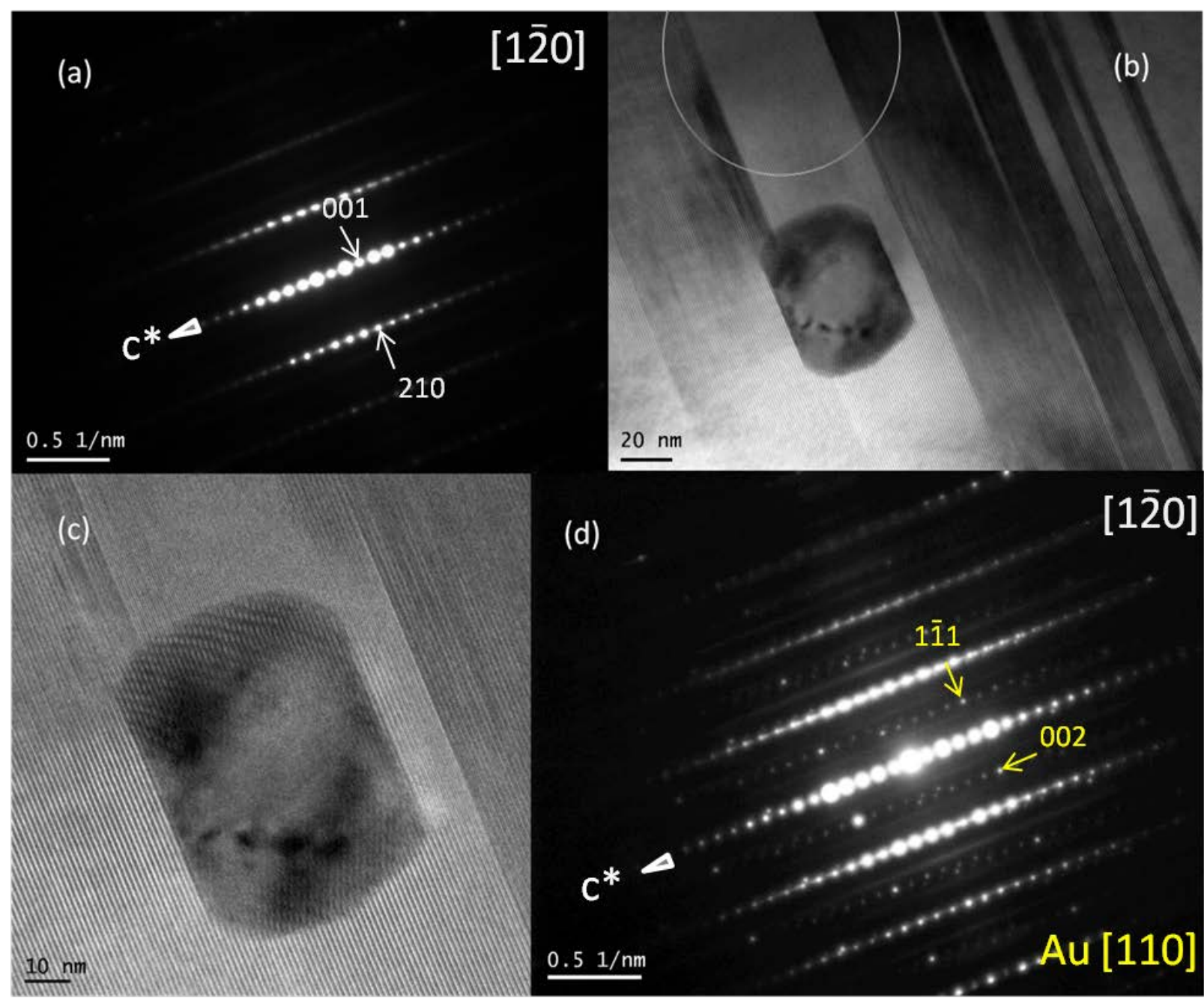

Figure 55. High resolution TEM images of the CCO - Au1\% samples. (a) diffraction pattern of marked area in (b) only CCO; the diffraction pattern is taken along the [1 $\overline{2}_{0}$ ] zone axis. (c) Au nanoparticle coherent with the Cco matrix. (d) diffraction pattern of the Au nanoparticle and CCO matrix taken from the centre of image (c) the zone axis for the CCO is [1 $\overline{2}_{0}$ ] and for the $\mathrm{Au}$ is [110]. 


\subsection{Transport Properties}

The transport properties for the CCO - Au\% composites are shown in Figure 56 . There is temperature dependence of all properties, however only slight changes of resistivity are observed through the temperature range.

The addition of $\mathrm{Au}$ is lessens the Seebeck coefficient for most of composite percentages studied. For the Au10\% composite, greater Seebeck coefficient values are obtained from about $900 \mathrm{~K}$ to $1100 \mathrm{~K}$. The maximum value is registered for the Au10\% composite at $1030 \mathrm{~K}$. All composite systems show a converging tendency for the Seebeck coefficient at $1100 \mathrm{~K}$. Upon large accumulation of metallic particles, degradation of the Seebeck coefficient occurs. [47] However in the CCO - Au systems obtained, the particles are rather small and well dispersed. Further understanding of this phenomenon is required.

Electrical resistivity is visibly affected by Au addition. For Au compositions of $1 \%$ and $2 \%$ decrease of the resistivity is observed over the entire temperature range whereas for Au compositions of $5 \%$ and $10 \%$ the electrical resistivity is increased, in detriment of the thermoelectric performance. The reduction of the resistivity is commonly associated to improved alignment; the added metallic particles are expected to facilitate carrier mobility between CCO grains.[47] From the cross section images it is clear that such alignment improvement is not accomplished and therefore there is no reduction of the electrical resistivity due to the texturing of the samples.

The power factor accounts for the combined effect of the Seebeck coefficient and the electrical resistivity. Interestingly, addition of Au nanoinclusions in concentration of $2 \%$ seems to have almost no effect on the power factor; in contrast to all other compositions that clearly do. For composites with Au1\% the power factor is enhanced whereas for $5 \%$ and $10 \%$ reduction of the power factor is observed.

Au nanoinclusions addition to CCO in concentrations of $5 \%$ and $10 \%$ has a decreasing effect on the thermal conductivity. Particularly for Au $10 \%$ there is a significant reduction of $15 \%$ from the baseline and $\mathrm{Au} 5 \%$ lowest values (at $973 \mathrm{~K}$ ). On the other hand, Au concentrations of $1 \mathrm{wt} \%$ and $2 \mathrm{wt} \%$ increase the thermal conductivity. 

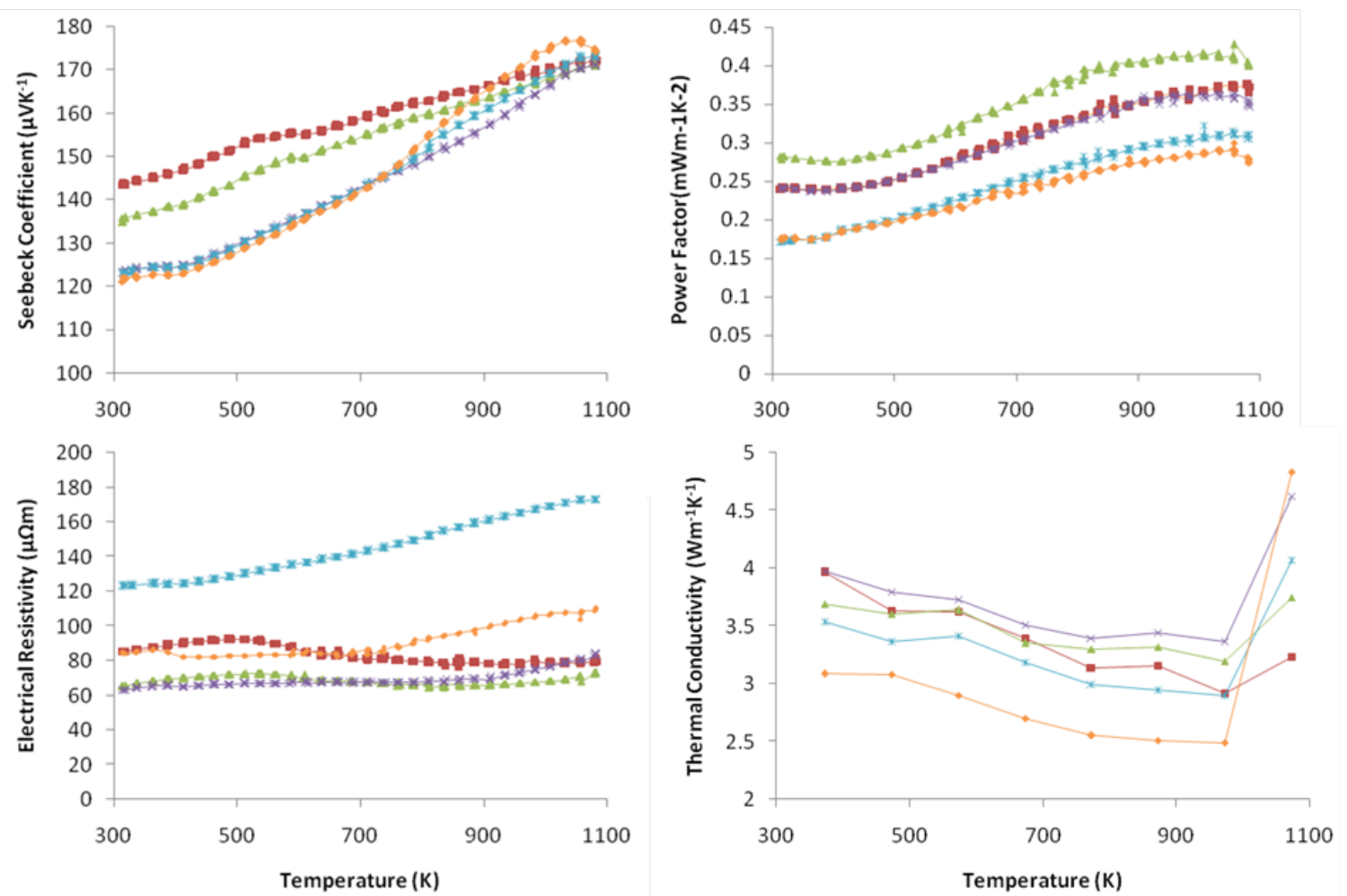

- Baseline $\longrightarrow$ Au $1 \% \multimap$ Au 2\% $\longrightarrow$ Au 5\% $\longrightarrow$ Au 10\%

Figure 56. Transport properties for the baseline and CCO - Au \% composites. Au wt\% concentrations of $1 \%, 2 \%, 5 \%$ and $10 \%$.

The behavior of the dimensionless figure of merit ZT for the baseline and the CCO - Au wt\% composites is shown in Figure 57. Very slight enhancement of ZT is obtained for CCO - Au1\% composites from room temperature to $1000 \mathrm{~K}$, however no significant improvement of the thermoelectric performance is observed. For all other Au wt\% concentration composites ZT is diminished. 


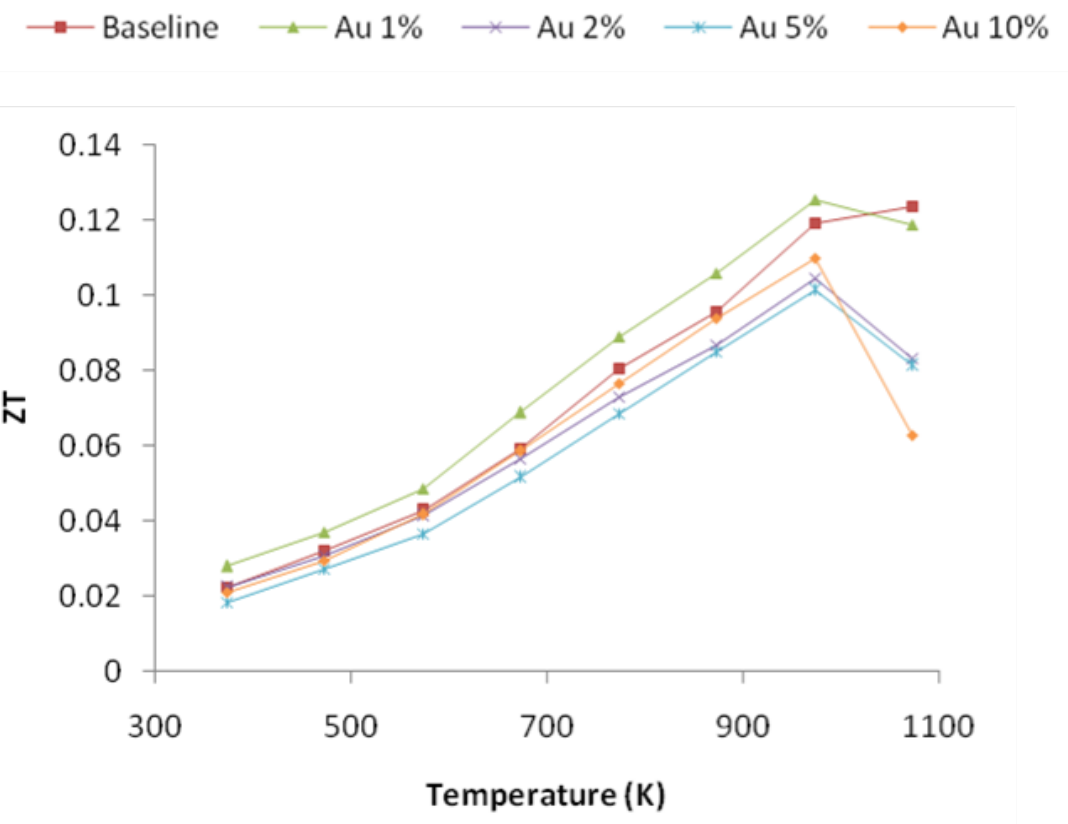

Figure 57. Dimensionless figure of merit for the baseline and CCO - Au \% composites. Au wt\% concentrations of $1 \%, 2 \%, 5 \%$ and $10 \%$. 


\subsection{Conclusions for the Chapter}

The CCO - Au samples prepared exhibit crystal textured characteristics as evidenced from XRD spectrums, however no visible change in the texture with increasing Au content was observed.

The density of the CCO - Au composites decreases as larger percentages of $\mathrm{Au}$ nanoparticles are introduced to the system.

Enormous growth of the Au nanoparticles takes place during the sample sintering process; ways to control such growth in order to avoid or retard such phenomena should be studied.

High coherence between the Au metallic particles and the CCO planes exist. The inclusions are found to be present at the interior of the grains as well as in the grain boundaries and between CCO lamellae within the same CCO grain.

The inclusion of $\mathrm{Au}$ nanoparticles in concentrations above $2 \%$ causes increment of the electrical resistivity with undesired effects on the thermoelectric performance. The power factor is only enhanced for Au 1\% composites; it could be possible that the optimum amount of Au nanoinclusions is even lower than this concentration and experimentation with lower Au quantities is suggested.

Addition of Au particles reduces the thermal conductivity; particularly for Au concentrations of $10 \%$ a reduction of $15 \%$ is accomplished.

$\mathrm{Au}$ addition as nanoinclusions in the CCO system in concentrations greater than $1 \%$ seems to have prejudicial effects on the thermoelectric performance evaluated through the dimensionless figure of merit ZT. 


\section{CHAPTER 6. Conclusions and Future Work}

\subsection{Conclusions}

The effect of the Bi doping on the thermoelectric properties and microstructure of the CCO pellets were systematically studied.

1. Ca site: The addition of Bi to the CCO system substituting for the Ca site improves the thermoelectric performance. Increasing doping level up to 0.3 concentration result in crystal texture development: grain growth, higher density and better alignment; for higher concentrations detriment of the thermoelectric performance occurs. The electrical resistivity and the thermal conductivity are reduced with increasing $\mathrm{Bi}$ doping level up to 0.3 concentration. For higher concentrations the electrical resistivity is significantly increased and the thermal conductivity is also increased.

2. Ca and Co sites: $\mathrm{Bi}$ addition to CCO substituting simultaneously for the $\mathrm{Ca}$ and $\mathrm{Co}$ sites in the composition $\mathrm{Ca}_{(3-x)} \mathrm{Bi}_{(x+y)} \mathrm{Co}_{(4-y)} \mathrm{O}_{9}$ with $\mathrm{x}=0.2$ and $\mathrm{y}=0.1$ improve the thermoelectric performance of the CCO system. The electric resistivity is reduced, the Seebeck coefficient is increased and the thermal conductivity is slightly reduced. The highest ZT value obtained was 0.32 at $973 \mathrm{~K}$ for the $0.5 \mathrm{GPa}$ and $25^{\circ} \mathrm{C}$ pressing conditions; corresponding to an improvement of $113 \%$ over the baseline better performance. Microstructure features were identified as evidence of the instability of the obtained compound. Secondary phase grains identified as cobalt oxide were found suggesting that preferred $\mathrm{Bi}$ substitution of $\mathrm{Ca}$ atoms in the CCO system occurs. Further study of the solubility limits and optimum doping level on the Co site is proposed.

3. The thermal conductivity affected by porosity of the CCO compounds. Careful selection of the manufacturing process will allow for optimum thermoelectric performance of the materials. It was observed that higher density of the compounds from higher pressure and temperature forming conditions not necessarily result in better performance due that the gain in electrical conductivity was accompanied by increased thermal conductivity. The milder forming conditions resulted in lower thermal conductivity values, allowing for higher ZT values. 
4. The optimum doping level of Bi substituting for the Ca site in the CCO system is 0.3 for which the ZT obtained is 0.22 at $1073 \mathrm{~K}$ for the $0.5 \mathrm{GPa}$ and $25{ }^{\circ} \mathrm{C}$ pressing conditions. The corresponding improvement is of $29 \%$ over the baseline better performance.

5. Dislocations at grain boundaries are preferred segregation zones for impurities with effect on the transport properties. Bi accumulation in such regions was demonstrated.

Au nanoinclusions were successfully induced into the CCO grains

1. High coherent Au metallic nanoinclusions were induced to the CCO system; the Au particles were at the interior of the grains, in the grain boundaries and between CCO lamellae within the CCO grains.

2. Decrease of the density of the pressed pellets with increasing Au nanoinclusions content was observed; accordingly reduction on the thermal conductivity was achieved. However great growth of the Au nanoparticles occurs during the sample sintering process and the reduction of thermal conductivity may as well be attributed to the increasing porosity with increasing Au \% content.

3. Reduction of the electrical resistivity was achieved for Au concentrations below $2 \%$, the power factor was improved for the $1 \%$ Au nanoparticles concentration composite. The optimum Au nanoinclusions concentration level is likely to be below $1 \mathrm{wt} \%$. The highest ZT Obtained is 0.125 at $973 \mathrm{~K}$ which corresponds to an improvement of the $1.6 \%$ over the baseline better performance.

The figure of merit behavior for the best performing materials among the systems studied are summarized in Figure 58. 
* $\mathrm{x}=0.3$ - Ca site Bi doping (0.5 GPa)

$\rightarrow-x=0.2$ - Ca site and $y=0.1$ - Co site Bi doping (0.5 GPa)

^Au $1 \%$ nanoinclusions (1 GPa)

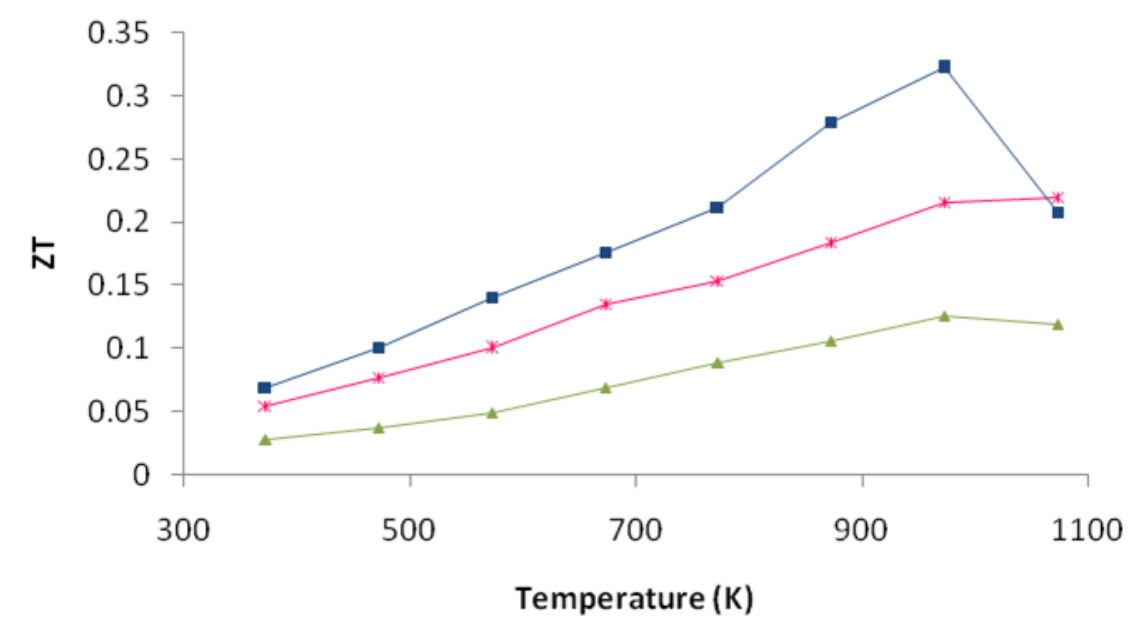

Figure 58. Dimensionless figure of merit for the best performing CCO materials among the systems studied. 


\subsection{Future Work}

The present work clearly demonstrated that $\mathrm{Bi}$ doping is effectively enhancing the overall energy conversion efficiency of the CCO system. The previous results from our group also demonstrate that $\mathrm{Yb}$ doping is increasing the electrical conductivity and decreasing the thermal conductivity simultaneously. Upon the successful attempt of $\mathrm{Bi}$ and $\mathrm{Yb}$ doping respectively, the co-doping of $\mathrm{Yb}$ and $\mathrm{Bi}$ on the $\mathrm{Ca}$ site simultaneously will be performed and evaluated.

The Bi solubility limits for the Co site and optimum doping levels for the simultaneous $\mathrm{Ca}$ and Co substitution case will be studied.

The Au nanoinclusions were successfully induced into the CCO grains. However, to effectively reduce the thermal conductivity of the sample, the Au particles need to be with smaller grain size and increased density of the dispersion in the CCO grains. Materials processing to decrease the Au particles size and increase the particles density will be optimized, and the effect of thermoelectric performance of CCO with various Au nanoinclusions will be evaluated.

Combination of mechanisms (doping and nanoinclusions) for tuning the transport properties should also be studied to understand any joint effect over the CCO system. 


\section{Acknowledgements}

Special thanks to my academic advisor Dr. Xueyan Song, and to Dr. Yun Chen and Diego Palacio for their continuous collaboration and suggestions. I would also like to thank all those involved with this research for their help and support. Special thanks to the committee members for their time and valuable opinion.

I would also like to thank WVU for giving me the opportunity to form part of their community and providing me not only with very high quality professors and scholars but very good friends. I want also to thank the Office of International Students and Scholars for their labor which helped feel WVU a second home.

Finally I would like to thank my family for their continuous advice and accompaniment throughout every stage in my life and particularly during this research time. 


\section{References}

[1] M. Shikano, R. Funahashi, Applied Physics Letters 82 (2003) 1851-1853.

[2] H.J. Goldsmid, Introduction to Thermoelectricity, 2009.

[3] D.M. Rowe, Renewable Energy 16 (1999) 1251-1256.

[4] D.M. Rowe, Thermoelectrics handbook macro to nano, CRC/Taylor \& Francis, Boca Raton, 2006.

[5] D.M. Rowe, CRC handbook of thermoelectrics, CRC Press, Boca Raton, FL, 1995.

[6] M. Ohtaki, Journal of the Ceramic Society of Japan 119 (2011) 770-775.

[7] M. Ohtaki, T. Tokunaga, K. Eguchi, H. Arai, leee, Proceedings Ict'97 - Xvi International Conference on Thermoelectrics (1997) 224-227.

[8] H. Ohta, S. Kim, Y. Mune, T. Mizoguchi, K. Nomura, S. Ohta, T. Nomura, Y. Nakanishi, Y. Ikuhara, M. Hirano, H. Hosono, K. Koumoto, Nature Materials 6 (2007) 129-134.

[9] M. Ohtaki, K. Araki, K. Yamamoto, Journal of Electronic Materials 38 (2009) 1234-1238.

[10] D.L. Wang, L.D. Chen, Q. Wang, J.G. Li, Journal of Alloys and Compounds 376 (2004) 58-61.

[11] I. Terasaki, Y. Sasago, K. Uchinokura, Physical Review B 56 (1997) 12685-12687.

[12] K. Fujita, T. Mochida, K. Nakamura, Japanese Journal of Applied Physics Part 1-Regular Papers Short Notes \& Review Papers 40 (2001) 4644-4647.

[13] A.C. Masset, C. Michel, A. Maignan, M. Hervieu, O. Toulemonde, F. Studer, B. Raveau, J. Hejtmanek, Physical Review B 62 (2000) 166-175.

[14] I. Terasaki, M. Iwakawa, T. Nakano, A. Tsukuda, W. Kobayashi, Dalton Transactions 39 (2010) 10051011.

[15] Y. Wang, Y. Sui, X. Wang, W. Su, X. Liu, Journal of Applied Physics 107 (2010).

[16] R. Funahashi, S. Urata, T. Sano, M. Kitawaki, Journal of Materials Research 18 (2003) 1646-1651.

[17] D. Kenfaui, G. Bonnefont, D. Chateigner, G. Fantozzi, M. Gomina, J.G. Noudem, Materials Research Bulletin 45 (2010) 1240-1249.

[18] D. Kenfaui, D. Chateigner, M. Gomina, J.G. Noudem, Journal of Alloys and Compounds 490 (2010) 472-479.

[19] H.Y. Choi, M.H. Lee, S.M. Choi, W.S. Seo, H.L. Lee, Journal of Ceramic Processing Research 13 (2012) S114-S118.

[20] C.H. Lim, S.M. Choi, W.S. Seo, H.H. Park, Journal of Electronic Materials 41 (2012) 1247-1255.

[21] C.H. Lim, S.M. Choi, W.S. Seo, K.H. Kim, J.Y. Kim, H.H. Park, Journal of Ceramic Processing Research 13 (2012) 197-201.

[22] J. Xu, C. Wei, K. Jia, Journal of Alloys and Compounds 500 (2010) 227-230.

[23] R. Funahashi, I. Matsubara, H. Ikuta, T. Takeuchi, U. Mizutani, S. Sodeoka, Japanese Journal of Applied Physics Part 2-Letters 39 (2000) L1127-L1129.

[24] M. Mikami, N. Ando, E. Guilmeau, R. Funahashi, Japanese Journal of Applied Physics Part 1-Regular Papers Brief Communications \& Review Papers 45 (2006) 4152-4158.

[25] S. Katsuyama, Y. Takiguchi, M. Ito, Journal of Materials Science 43 (2008) 3553-3559.

[26] Y. Miyazaki, Solid State Ionics 172 (2004) 463-467.

[27] G.J. Xu, R. Funahashi, M. Shikano, I. Matsubara, Y.Q. Zhou, Applied Physics Letters 80 (2002) 3760-

3762.

[28] S.W. Li, R. Funahashi, I. Matsubara, K. Ueno, S. Sodeoka, H. Yamada, Chemistry of Materials 12 (2000) 2424-2427.

[29] Y. Liu, Y. Lin, L. Jiang, C.-W. Nan, Z. Shen, Journal of Electroceramics 21 (2008) 748-751. 
[30] N.V. Nong, C.J. Liu, M. Ohtaki, Journal of Alloys and Compounds 509 (2011) 977-981.

[31] D.L. Wang, L.D. Chen, Q. Yao, J.G. Li, Solid State Communications 129 (2004) 615-618.

[32] P. Che, Y. Zhang, Q.B. Bo, J. Feng, J.P. Wang, J. Meng, Journal of Rare Earths 22 (2004) 165-167.

[33] H.Q. Liu, Y. Song, S.N. Zhang, X.B. Zhao, F.R. Wang, Journal of Physics and Chemistry of Solids 70 (2009) 600-603.

[34] X. Song, Y. Chen, S. Chen, E. Barbero, E.L. Thomas, P. Barnes, Solid State Communications 152

(2012) 1509-1512.

[35] D.L. Wang, L.D. Chen, S.Q. Bai, J.G. Li, Journal of Inorganic Materials 19 (2004) 1329-1333.

[36] Y. Wang, Y. Sui, J.G. Cheng, X.J. Wang, W.H. Su, Journal of Alloys and Compounds 477 (2009) 817-

821.

[37] G.D. Tang, C.P. Tang, X.N. Xu, Y. He, L. Qiu, L.Y. Lv, Z.H. Wang, Y.W. Du, Journal of Electronic Materials 40 (2011) 504-507.

[38] M. Mikami, K. Chong, Y. Miyazaki, T. Kajitani, T. Inoue, S. Sodeoka, R. Funahashi, Japanese Journal of Applied Physics Part 1-Regular Papers Brief Communications \& Review Papers 45 (2006) 4131-4136.

[39] N.V. Nong, S. Yanagiya, S. Monica, N. Pryds, M. Ohtaki, Journal of Electronic Materials 40 (2011) 716-722.

[40] J.L. Chen, Y.S. Liu, C.J. Liu, L.C. Huang, C.L. Dong, S.S. Chen, C.L. Chang, Journal of Physics D-Applied Physics 42 (2009).

[41] Y. Miyazaki, K. Kudo, M. Akoshima, Y. Ono, Y. Koike, T. Kajitani, Japanese Journal of Applied Physics Part 2-Letters 39 (2000) L531-L533.

[42] H. Yakabe, K. Kikuchi, I. Terasaki, Y. Sasago, K. Uchinokura, leee, Proceedings Ict'97 - Xvi International Conference on Thermoelectrics (1997) 523-527.

[43] Y. Miyazaki, Y. Suzuki, M. Onoda, Y. Ishii, Y. Morii, T. Kajitani, Japanese Journal of Applied Physics Part 1-Regular Papers Short Notes \& Review Papers 43 (2004) 6252-6258.

[44] H.Q. Liu, X.B. Zhao, T.J. Zhu, Y. Song, F.P. Wang, Current Applied Physics 9 (2009) 409-413.

[45] P.H. Xiang, Y. Kinernuchi, H. Kaga, K. Watari, Journal of Alloys and Compounds 454 (2008) 364-369.

[46] N. Ngo Van, N. Pryds, S. Linderoth, M. Ohtaki, Advanced Materials 23 (2011) 2484-+.

[47] M. Mikami, N. Ando, R. Funahashi, Journal of Solid State Chemistry 178 (2005) 2186-2190.

[48] Y. Wang, Y. Sui, J. Cheng, X. Wang, W. Su, Journal of Physics D-Applied Physics 41 (2008).

[49] M. Ito, D. Furumoto, Journal of Alloys and Compounds 450 (2008) 494-498.

[50] J.W. Fergus, Journal of the European Ceramic Society 32 (2012) 525-540.

[51] Y. Song, Q. Sun, L. Zhao, F. Wang, Z. Jiang, Materials Chemistry and Physics 113 (2009) 645-649.

[52] I. Matsubara, R. Funahashi, M. Shikano, K. Sasaki, H. Enomoto, Applied Physics Letters 80 (2002)

4729-4731.

[53] K. Koumoto, Y. Wang, R. Zhang, A. Kosuga, R. Funahashi, Annual Review of Materials Research, Vol 4040 (2010) 363-394.

[54] N. Van Nong, N. Pryds, S. Linderoth, M. Ohtaki, Advanced materials (Deerfield Beach, Fla.) 23 (2011) 2484-2490.

[55] D.M. Rowe, Materials Preparation and Characterization in Thermoelectrics (2012).

[56] K. Byrappa, M. Yoshimura, Handbook of Hydrothermal Technology - A Technology for Crystal Growth and Materials Processing, William Andrew Publishing/Noyes, 2001.

[57] C.B. Carter, M.G. Norton, Ceramic Materials: Science and Engineering, Springer, 2007.

[58] J.P. Singh, N.P. Bansal, J. Lamon, M.M. Mahmoud, S.R. Choi, Processing and Properties of Advanced Ceramics and Composites II, Wiley, 2010.

[59] TPN - 68 Thermal Diffusivity by the Laser Flash Method.

[60] D.A. Muller, Nature Materials 8 (2009) 263-270.

[61] S. Chen, X.Y. Song, X.Q. Chen, Y. Chen, E.J. Barbero, E.L. Thomas, P.N. Barnes, Journal of Sol-Gel Science and Technology 64 (2012) 627-636. 